NUCLEAR ENERGY RESEARCH INITIATIVE (NERI)

FINAL REPORT

Award Number DE-FC07-07ID14827

\title{
OPTIMIZATION OF HEAT EXCHANGERS
}

\author{
$\underline{\text { Principal Investigator }}$ \\ Ivan Catton, Professor of Engineering \\ Department of Mechanical and Aerospace Engineering \\ School of Engineering and Applied Science \\ University of California, Los Angeles \\ Los Angeles, California 90095 \\ Submitted on 1 October, 2010
}




\section{Executive Summary}

The objective of this research is to develop tools to design and optimize heat exchangers (HE) and compact heat exchangers (CHE) for intermediate loop heat transport systems found in the very high temperature reactor (VHTR) and other Generation IV designs by addressing heat transfer surface augmentation and conjugate modeling. To optimize heat exchanger, a fast running model must be created that will allow for multiple designs to be compared quickly. To model a heat exchanger, volume averaging theory, VAT, is used. VAT allows for the conservation of mass, momentum and energy to be solved for point by point in a 3 dimensional computer model of a heat exchanger. The end product of this project is a computer code that can predict an optimal configuration for a heat exchanger given only a few constraints (input fluids, size, cost, etc.). As VAT computer code can be used to model characteristics (pumping power, temperatures, and cost) of heat exchangers more quickly than traditional CFD or experiment, optimization of every geometric parameter simultaneously can be made. Using design of experiment, DOE and genetic algorithms, GE, to optimize the results of the computer code will improve heat exchanger design.

For optimization to be accomplished, one needs to separate conduction and convection at its natural interface. This means friction and heat transfer at the interface needs to be treated in a way that allows each side to be independently varied. If you increase the flow rate, the heat transfer coefficient will increase and you need to take advantage of this by changing the geometry to modify the conduction side of the problem. Of course you try to do this while keeping the friction factor as small as possible. All of this is done within a complex heterogeneous hierarchical structure. 
Several issues arise when we set out to solve such a problem. One of the more interesting is how you compare CFD calculations to measured values of the two parameters that are needed. Another is how to directly use experimental data. The simple equations contain coefficients that are essentially averages of some kind and we are usually unsure of what they are. Measured friction factors are fairly straightforward to interpret although calculated values are not. Measured heat transfer coefficients are usually based on some kind of upper level assumptions and often not what we need and calculated values can be difficult to interpret correctly. Using VAT to derive the simple equations leads us to a rigorous definition of the needed coefficients whether obtained from experiment or through the use of CFD.

Determination of flow-variables and scalar transport in a heterogeneous (or porous) media is difficult even when subject to simplifications allowing the specification of medium periodicity or regularity. Linear or linearized models fail to intrinsically account for transport phenomena, requiring dynamic coefficient models to correct for short-comings in the governing models. Allowing inhomogeneities to adopt random or stochastic character further confounds the already daunting task of properly identifying pertinent transport mechanisms and predicting transport phenomena, see Travkin and Catton [1999].

Some aspects of the development of transport phenomena in porous media are now well understood and have seen substantial progress in thermal physics and fluid mechanics sciences, particularly in porous media transport phenomena. The basis for this progress is Volume Averaging Theory (VAT) first proposed in the sixties by Anderson and Jackson [1967], Whitaker [1967] and others. Many of the important details and examples of its application are found in books by Kheifets and Neimark [1982] and most recently by Whittaker [1999]. 
In most physically realistic cases, highly complex integral-differential equations result. The largest challenge is the insufficient development of closure theory, especially for integraldifferential equations. The ability to accurately evaluate various kinds of medium morphology irregularities results from the modelling methodology once a porous medium morphology is assigned. Further, when attempting to describe transport processes in a heterogeneous media, the correct form of the governing equations remains an area where inattention to procedure by some researchers has led to significantly different equations for the same media (see Travkin and Catton $[1992,1998])$.

The technical objectives of the proposed research are to: Develop a surface that increases surface heat removal effectiveness by a 4 without a marked increase in pumping power, Develop a new type of model capable of predicting flow and heat transfer in 2D and 3D spatial structures at different scales, Design and carry out experiments to measure heat transfer augmentation and validate the HE and CHE, Design and test a printed circuit heat exchanger for a high-temperature inert gas cycle.

The overall goal of the project is to develop a tool that can optimize a heat exchanger for given constraints quickly. The complete VAT tool with an included genetic algorithm will be able to predict the cheapest heat exchanger for any application. Using the experimental and CFD approaches to determine heat transfer and drag correlations for irregular porous media, more geometries can be analyzed and optimized. 


\section{Contents}

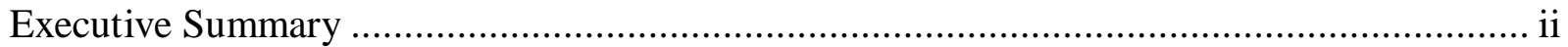

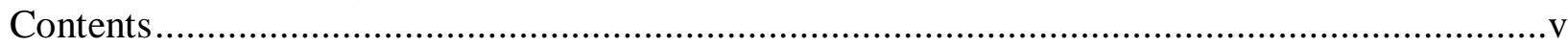

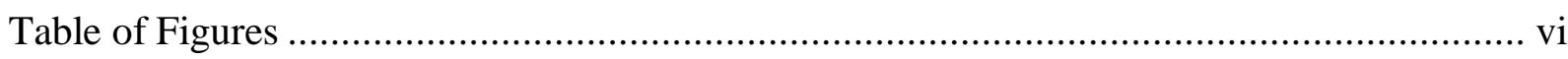

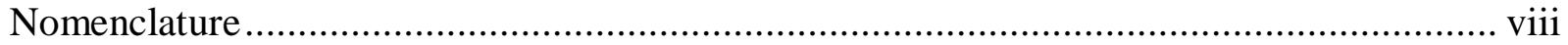

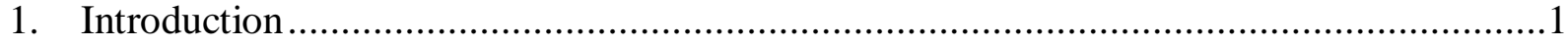

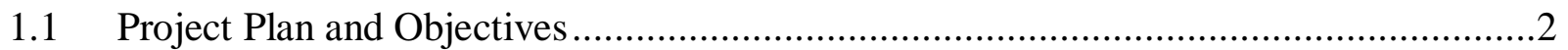

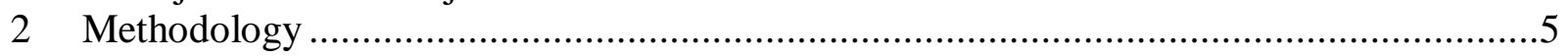

$2.1 \quad$ Fundamentals of VAT ............................................................................

2.2 VAT Based Equations in Heterogeneous Media ................................................. 10

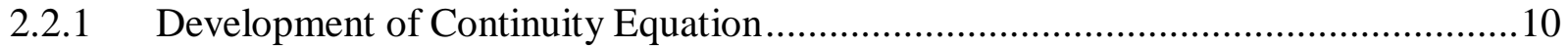

2.2.2 Development of Momentum Equation ............................................................. 11

2.2.3 Development of Turbulent Kinetic Energy Equation............................................ 12

2.2.4 Development of Energy Equations.......................................................... 13

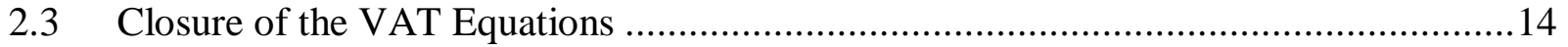

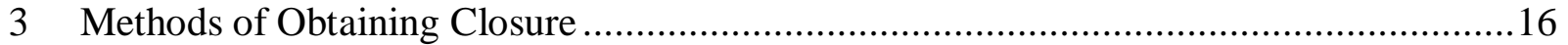

3.1 Closure Using Experimental Results ..................................................................2 21

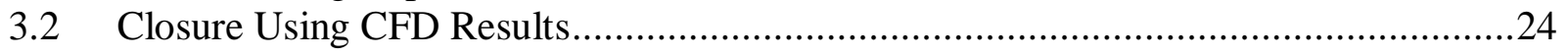

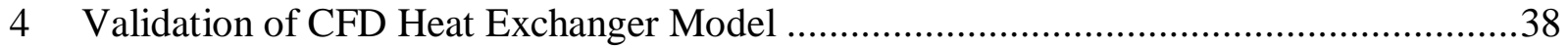

4.1 Parameter Definitions and Data Reduction ..............................................................39

4.2 Experimental Determination of Heat Transfer ....................................................49

4.3 Heat Exchanger Optimization Modeling...........................................................54

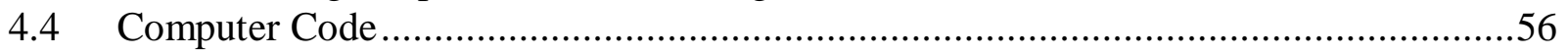

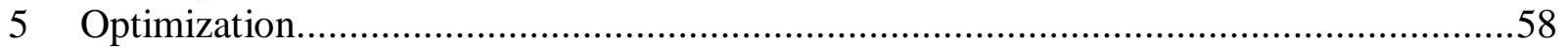

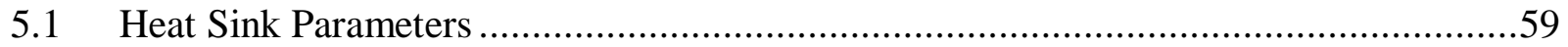

5.2 Design of Experiment ...........................................................................62

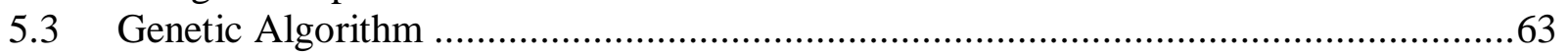

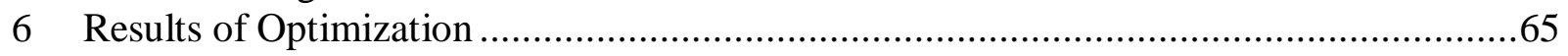

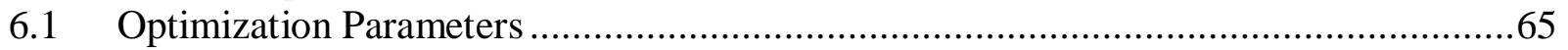

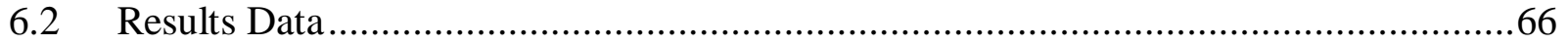

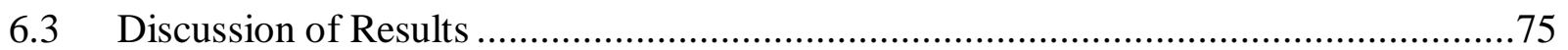

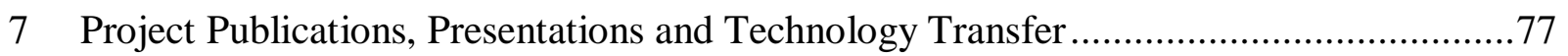

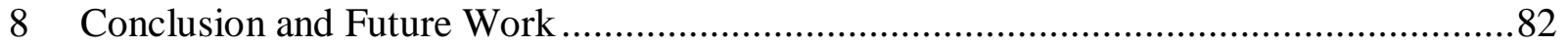

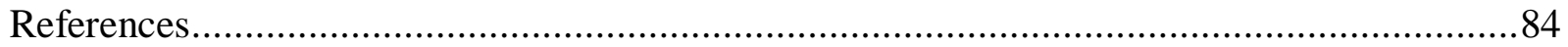




\section{Table of Figures}

Figure 1: Typical fin tube heat exchanger...................................................................................1

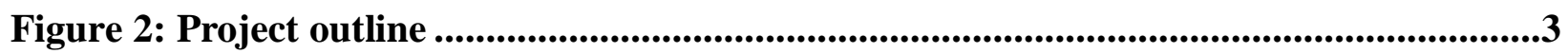

Figure 3: Representative Elementary Volume (REV) ...................................................9

Figure 4: REV for a Finned Tube Heat Exchanger ............................................................22

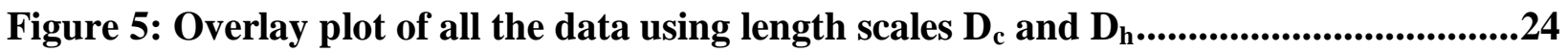

Figure 6: Comparison of heat transfer coefficients using $D_{c}$ and $D_{h}$ as the length scales.....26

Figure 7: Computational domain morphology .............................................................27

Figure 8: Comparison of VAT closure term $\mathrm{c}_{\mathrm{d}}$ by CFD to data ..........................................27

Figure 9: Contribution of viscous and pressure forces to the drag resistance coefficient ....28

Figure 10: Comparison of VAT closure Nu obtained by CFD with experiment ..................30

Figure 11: Development of averaged temperatures of fluid and solid phase at $R_{h}=31.2 \ldots . .31$

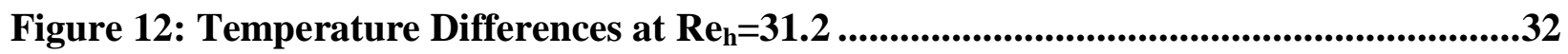

Figure 13: Local Heat Transfer Coeficient at $\mathrm{Re}_{\mathrm{h}}=31.2$........................................................32

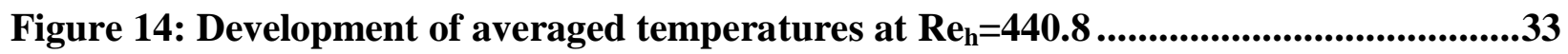

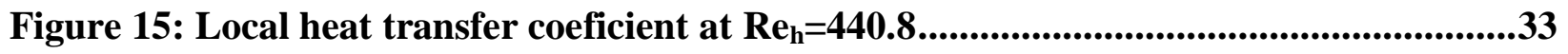

Figure 16: Local heat transfer coefficient with different types of heating. ............................34

Figure 17: Comparison of CFD results with experimental data .......................................35

Figure 18: Local heat transfer coefficient at different flow rates from Zhou [2010]............36

Figure 19: A schematic diagram of a plain plate finned tube heat exchanger......................38

Figure 20: Grid system for CFD analysis .............................................................................39

Figure 21: Comparison between CFD results, correlations and experimental data..............43

Figure 22: Variation of $N u$ and $f$ with tube row number by CFD simulation. .....................44

Figure 23: Variation of $M$ and $\theta_{\mathrm{m}}$ with tube row number $N$. ...........................................45

Figure 24: N=2 Temperature, Velocity and Intersection angle profiles...............................46 
Figure 25: N=9 Temperature, Velocity and Intersection angle profiles....................................46

Figure 26: Variation of $M, N u$ and $\theta_{\mathrm{m}}$ of each REV. .................................................................48

Figure 27: Experimental section for bed of spheres .......................................................................50

Figure 28: Packed bed of spheres test section ...................................................................................51

Figure 29: Dimensionless gas exit temperature versus time, with varying $\mathrm{Nu}$.......................52

Figure 30: Example of a Transient Gas Phase Temperature Response ...................................53

Figure 31: Gas Phase Temperature Response: Experimental Versus Simulation..................53

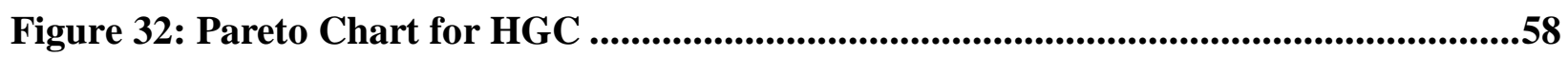

Figure 33: REV with 6 of the geometry parameters ........................................................................60

Figure 34: A cost function of the heat exchanger $C_{h x}$ and the source data ..........................62

Figure 35: Design of Experiment Flow chart .....................................................................................63

Figure 36: Flow diagram for genetic algorithm .............................................................................66

Figure 37: The effect of flow rate versus tube diameter .............................................................68

Figure 38: Flow rate versus Fin pitch......................................................................................................69

Figure 39: effect of flow rate versus fin thickness ............................................................70

Figure 40: Effect of length versus air flow rate..............................................................................71

Figure 41: Effect of Tube pitch in the $x$ direction versus air flow .............................................72

Figure 42- Effect pitch of tubes in y direction versus air flow.....................................................73

Figure 43: Effect of number of tubes in y direction versus airflow.............................................74

Figure 44: Effect of number of tubes in the $x$ direction versus the air flow rate ....................75 


\section{Nomenclature}

\section{Latin}

\begin{tabular}{|c|c|}
\hline$b$ & mean turbulent fluctuation energy $\left[\mathrm{m}^{2} / \mathrm{s}^{2}\right]$ \\
\hline$c_{d}$ & mean drag resistance coefficient in the REV [-] \\
\hline$c_{d p}$ & mean form resistance coefficient in the REV [-] \\
\hline$c_{f L}$ & mean skin friction coefficient over the laminar region in the REV [-] \\
\hline$c_{p}$ & specific heat $[\mathrm{J} /(\mathrm{kgK})]$ \\
\hline$C_{D}$ & empirical coefficient [-] \\
\hline$C_{\mu}$ & empirical coefficient [-] \\
\hline$D_{\mathrm{p}}$ & tube diameter $[\mathrm{m}]$ \\
\hline$\partial S_{w}$ & internal surface in the REV $\left[\mathrm{m}^{2}\right]$ \\
\hline Eff & effectiveness $[1 / \mathrm{K}]$ \\
\hline HTEFF & Ratio of heat transferred between fluids to theoretical max. \\
\hline$f$ & friction factor $[-]$ \\
\hline$g$ & gravitational constant $\left[1 / \mathrm{m}^{2}\right]$ \\
\hline$h$ & height of roughness element $[\mathrm{m}]$ \\
\hline$h_{b}$ & height of base plate $[\mathrm{m}]$ \\
\hline$h_{c}$ & height of channel [m] \\
\hline$k$ & thermal conductivity $[\mathrm{W} /(\mathrm{mK})]$ \\
\hline$k_{s T}$ & effective thermal conductivity of solid phase $[\mathrm{W} /(\mathrm{mK})]$ \\
\hline$k_{T}$ & turbulent eddy thermal conductivity $[\mathrm{W} /(\mathrm{mK})]$ \\
\hline$L$ & turbulence mixing length $[\mathrm{m}]$ \\
\hline$\langle m\rangle$ & average porosity [-] \\
\hline$\dot{m}$ & mass flow rate $[\mathrm{kg} / \mathrm{s}]$ \\
\hline$N u_{D}$ & Nusselt number based on diameter [-] \\
\hline$P$ & pressure $[\mathrm{Pa}]$ and pitch in regular porous $2 \mathrm{D}$ and $3 \mathrm{D}$ medium $[\mathrm{m}]$ \\
\hline$P x$ & Tube pitch in $\mathrm{x}$ direction \\
\hline Py & Tube pitch in y direction \\
\hline $\mathrm{Pr}$ & Prandtl number [-] \\
\hline$q_{w}$ & wall heat flux $\left[\mathrm{W} / \mathrm{m}^{2}\right]$ \\
\hline$\dot{Q}$ & heat transfer rate $[\mathrm{W}]$ \\
\hline$R e_{D}$ & Reynolds number based on diameter [-] \\
\hline$S_{w}$ & specific surface of a porous medium $\partial S_{w} / \Delta \Omega[1 / \mathrm{m}]$ \\
\hline$S_{\perp}$ & cross flow projected area of obstacles $\left[\mathrm{m}^{2}\right]$ \\
\hline$T$ & temperature $[\mathrm{K}]$ \\
\hline$u$ & velocity in $\mathrm{x}$ direction $[\mathrm{m} / \mathrm{s}]$ \\
\hline$v$ & velocity in y direction $[\mathrm{m} / \mathrm{s}]$ \\
\hline . & velocity in $\mathrm{z}$ direction $[\mathrm{m} / \mathrm{s}]$ \\
\hline
\end{tabular}

\section{Greek}

$\tilde{\alpha}_{T} \quad$ averaged heat transfer coefficient over $\partial S_{w}\left[\mathrm{~W} /\left(\mathrm{m}^{2} \mathrm{~K}\right)\right]$

$\Delta \Omega \quad$ representative elementary volume (REV) $\left[\mathrm{m}^{3}\right]$ 
$\Delta \Omega_{f} \quad$ pore volume in $\mathrm{REV}\left[\mathrm{m}^{3}\right]$

$\Delta \Omega_{s} \quad$ solid phase volume in REV [ $\left.\mathrm{m}^{3}\right]$

$\sigma_{b} \quad$ turbulent coefficient exchange ratio [-]

$v \quad$ kinematic viscosity $\left[\mathrm{m}^{2} / \mathrm{s}\right]$

$\rho \quad$ density $\left(\mathrm{kg} / \mathrm{m}^{3}\right)$

\section{Subscript}

$\begin{array}{ll}f & \text { fluid phase } \\ i & \text { component of turbulent vector variable } \\ L & \text { Laminar } \\ s & \text { solid phase } \\ T & \text { Turbulent } \\ w & \text { Wall }\end{array}$

\section{Superscript}

$\sim \quad$ value in fluid phase averaged over the REV

$\wedge \quad$ value in solid phase averaged over the REV

- mean turbulent quantity

' turbulent fluctuation quantity 


\section{Introduction}

By optimizing heat exchangers, savings in space and cost of materials, power and effectiveness can be useful. By increasing the effectiveness, less power is required to run the heat exchanger and less material is needed to fabricate it, both resulting in cost savings. Currently, heat exchangers being used generally have not been optimized by analyzing every variable that describes them simultaneously. In the case of a fin tube heat exchanger shown in Figure 1, this would be 11 parameters: overall length, width and height, internal fin thickness, fin pitch, tube diameter, tube wall thickness tube pitch in $\mathrm{x}$ and $\mathrm{y}$ directions, and flow rates of fluid 1 and 2 .

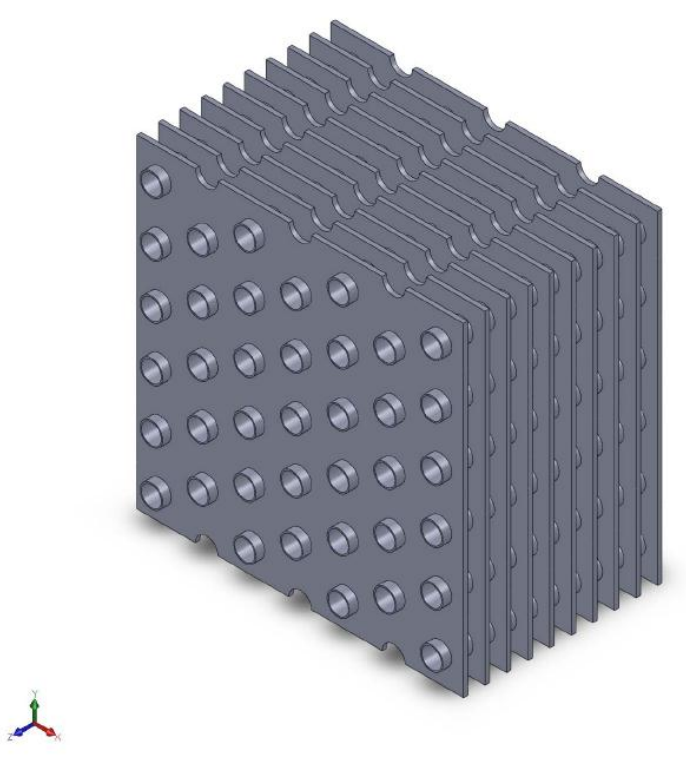

\section{Figure 1: Typical fin tube heat exchanger}

In the past, the emphasis was on experimental work due to absence of today's computational power. In the last 20 years, numerical simulation has been widely used to simulate the flow and heat transfer processes in heat exchangers for design and optimization purposes. However, experimental methods are expensive and time consuming, as many different models must be fabricated and tested. Numerical methods are more efficient, but many are specific to the type of geometry that is being tested and direct numerical simulation of the full $3 \mathrm{D}$ structure is 
often not feasible. Using Volume Averaging Theory (VAT) for flow and heat transfer, a more general model that can easily be adapted to many different structures can be made. By modeling heat exchangers as porous media, specific geometry can be accounted for in such a way that the details of the original structure can be replaced by their averaged counterparts and the governing VAT equations can be solved for a wide range of heat exchanger designs.

Proper closure is needed to complete the VAT governing equations. Travkin and Catton (1995, 1998) showed that choosing the correct length scale, which is found to be a hydraulic diameter of the porous media on theoretical basis, allows one to collapse true capillary flow and flow in a bed of spheres spanning the physical description from globular to capillary geometry with a single length scale. In this work, the VAT based equations are given and their closures are defined in terms of the resulting averages. The suggested scaling is then used to collapse experimental data for friction factor and heat transfer onto a single curve respectively. A much simpler correlation for friction factor and Nusselt number is proposed separately. Using these simplified forms for friction factor and Nusselt number, any porous media heat transfer problem can be solved with VAT theory faster than with CFD or by experiment and thus optimization of each of the 11 parameters of a heat exchanger is possible in a timely manner.

\subsection{Project Plan and Objectives}

By using VAT theory, the momentum and energy equations can be solved for an arbitrary heat exchanger. As Shown in Figure 2, the end product is a computer code that can predict an optimal configuration for a heat exchanger given only a few constraints. As this can be used to model characteristics of heat exchangers more quickly than CFD or experiment, optimization of every geometric parameter simultaneously can be made. Using design of experiment, DOE and 
genetic algorithms, GE, to optimize the results of the computer code will improve heat exchanger design.

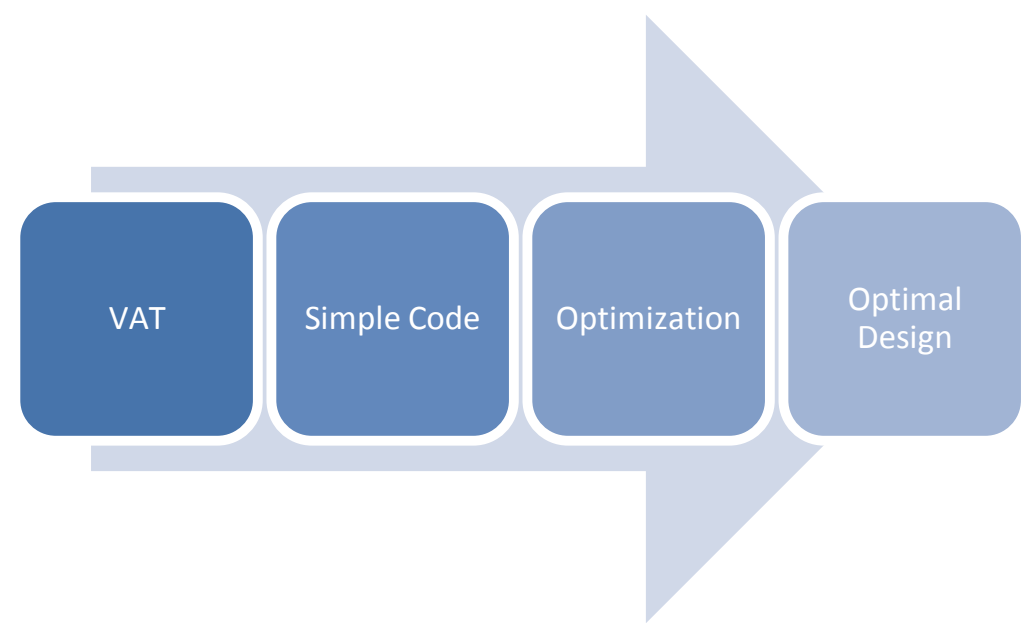

Figure 2: Project outline

To close the VAT equations needed to model the heat exchanger, data in the form of correlations for the local heat transfer and friction factor are needed. These can be found from experimental methods or from the use of CFD techniques. By using the proper scaling on these correlations, a simple form can be used that still includes all the effects of the geometry, as it is defined by the local porosity and wetted surface area.

The technical objectives of the proposed research are to:

1) Develop a surface that increases surface heat removal effectiveness by a 4 without a marked increase in pumping power,

2) Develop a new type of model capable of predicting flow and heat transfer in $2 \mathrm{D}$ and $3 \mathrm{D}$ spatial structures at different scales,

3) Design and carry out experiments to measure heat transfer augmentation and validate the HE and CHE, Design and

4) test a printed circuit heat exchanger for a high-temperature inert gas cycle. 
The overall goal of the project is to develop a tool that can optimize a heat exchanger for given constraints quickly. The complete VAT tool with an included genetic algorithm will be able to predict the cheapest heat exchanger for any application. Using the experimental and CFD approaches to determine heat transfer and drag correlations for irregular porous media, more geometries can be analyzed and optimized. 


\section{Methodology}

To illustrate the principles of a hierarchical modeling approach based on VAT developed to describe transport phenomena in ordered or disordered porous media, the geometry of a typical plate finned tube heat exchanger has been used. The general geometry arrangement of the heat exchanger is given in Figure 1.

For optimization to be accomplished, one needs to separate conduction and convection at its natural interface. This means friction and heat transfer at the interface needs to be treated in a way that allows each side to be independently varied. If you increase the flow rate, the heat transfer coefficient will increase and you need to take advantage of this by changing the geometry to modify the conduction side of the problem. Of course you try to do this while keeping the friction factor as small as possible. All of this is done within a complex heterogeneous hierarchical structure.

Several issues arise when we set out to solve such a problem. One of the more interesting is how you compare CFD calculations to measured values of the two parameters that are needed. Another is how to directly use experimental data. The simple equations contain coefficients that are essentially averages of some kind and we are usually unsure of what they are. Measured friction factors are fairly straightforward to interpret although calculated values are not. Measured heat transfer coefficients are usually based on some kind of upper level assumptions and often not what we need and calculated values can be difficult to interpret correctly. Using VAT to derive the simple equations leads us to a rigorous definition of the needed coefficients whether obtained from experiment or through the use of CFD. 
Determination of flow-variables and scalar transport in a heterogeneous (or porous) media is difficult even when subject to simplifications allowing the specification of medium periodicity or regularity. Linear or linearized models fail to intrinsically account for transport phenomena, requiring dynamic coefficient models to correct for short-comings in the governing models. Allowing inhomogeneities to adopt random or stochastic character further confounds the already daunting task of properly identifying pertinent transport mechanisms and predicting transport phenomena, see Travkin and Catton [1999].

Some aspects of the development of transport phenomena in porous media are now well understood and have seen substantial progress in thermal physics and fluid mechanics sciences, particularly in porous media transport phenomena. The basis for this progress is Volume Averaging Theory (VAT) first proposed in the sixties by Anderson and Jackson [1967], Whitaker [1967] and others. Many of the important details and examples of its application are found in books by Kheifets and Neimark [1982] and most recently by Whittaker [1999].

In most physically realistic cases, highly complex integral-differential equations result. The largest challenge is the insufficient development of closure theory, especially for integraldifferential equations. The ability to accurately evaluate various kinds of medium morphology irregularities results from the modelling methodology once a porous medium morphology is assigned. Further, when attempting to describe transport processes in a heterogeneous media, the correct form of the governing equations remains an area where inattention to procedure by some researchers has led to significantly different equations for the same media (see Travkin and Catton [1992, 1998]).

The VAT based approach has the following desirable features:

1. Effects of interfaces and boundaries can be included in the modeling. 
2. The effect of morphology of the different phases (solid, liquid and vapor) are incorporated. The morphology description is directly incorporated into the integro-differential field equations.

3. Separate and combined fields and their interactions are described exactly through the integral terms appearing in the field equations. No assumptions about effective transport coefficients are required.

4. Effective coefficients are rigorously derived whereas many present results are often simply wrong.

5. Deliberate design and optimization of materials using hierarchical physical descriptions based on the VAT governing equations can be used to connect properties and morphological characteristics to heat exchanger features.

\subsection{Fundamentals of VAT}

The method of volume averaging begins by associating an averaging volume $\Delta \Omega$ with every point in space. The averaging volume, the Representative Elementary Volume or REV, is illustrated in Fig. 1, and is represented in terms of the volumes of the individual phases by

$$
\Delta \Omega=\Delta \Omega_{f}(\vec{X}, t)+\Delta \Omega_{s}(\vec{X})
$$

The volumes of the fluid and solid phases can depend on position and, in addition, the volume of the fluid phase can depend on time. Average values are defined in terms of these volumes and these averaged values are associated with the centroid of $\Delta \Omega$. In the development of volume averaged transport equations, a superficial average and an intrinsic average are defined. The superficial average of $f$ is given by

$$
\left\langle f_{f}\right\rangle=\frac{1}{\Delta \Omega} \int_{\Delta \Omega_{f}} f_{f}(\vec{X}, t) d \omega,
$$

and the intrinsic average of $f$ is defined by

$$
\tilde{f}_{f}=\frac{1}{\Delta \Omega_{f}} \int_{\Delta \Omega_{f}} f_{f}(\vec{X}, t) d \omega
$$

The intrinsic and superficial averages are related by

$$
\left\langle f_{f}\right\rangle=\langle m\rangle \tilde{f}_{f}
$$

where $<\mathrm{m}>$ is the porosity in the averaging volume $\Delta \Omega$ defined by 


$$
\langle m\rangle=\frac{\Delta \Omega_{f}}{\Delta \Omega}
$$

The random porosity function in terms of the average value of $\langle m(\vec{x})\rangle$ in the REV and its fluctuations $\hat{m}(\vec{x})$ in various directions is

$$
m(\vec{x})=<m(\vec{x})+\hat{m}(\vec{x}) .
$$

The different types of averaging over the REV function $f$ are defined by the following averaging operators: the average of the function $f$ over the REV is given by

$$
\langle f\rangle=\frac{1}{\Delta \Omega} \int_{\Delta \Omega} f(\vec{X}, t) d \omega=\langle m\rangle \tilde{f}+(1-\langle m\rangle) \hat{f}
$$

the phase average of the function $\mathrm{f}$ in the fluid

$$
\langle f\rangle_{f}=\frac{\Delta \Omega_{f}}{\Delta \Omega} \frac{1}{\Delta \Omega_{f}} \int_{\Delta \Omega_{f}} f(\vec{X}, t) d \omega=\langle m\rangle \tilde{f},
$$

the phase average of the function $f$ in the solid phase

$$
\langle f\rangle_{s}=\frac{\Delta \Omega_{s}}{\Delta \Omega} \frac{1}{\Delta \Omega_{s}} \int_{\Delta \Omega_{s}} f(\vec{X}, t) d \omega=(1-\langle m\rangle) \hat{f},
$$

And the interphase average of each component is

$$
\begin{aligned}
& \tilde{f}=\{f\}_{f}=\frac{1}{\Delta \Omega_{f}} \int_{\Delta \Omega_{f}} f(\vec{X}, t) d \omega, \\
& \widehat{f}=\{f\}_{s}=\frac{1}{\Delta \Omega_{s}} \int_{\Delta \Omega_{s}} f(\vec{X}, t) d \omega .
\end{aligned}
$$

When the interface is fixed in space, averaged functions for the first and second phase within the REV and over the entire REV fulfill all four of the Reynolds conditions as well as the following four consequences

$$
\{\tilde{f}\}_{f}=\tilde{f},\{\tilde{f}\}_{f}=\{f-\tilde{f}\}_{f}=0,\{\tilde{f} \tilde{g}\}_{f}=\tilde{f} \tilde{g},\{\tilde{f} \hat{g}\}_{f}=\tilde{f} \tilde{\hat{g}}=0
$$

The differential operator average,

$$
\{\nabla f\}_{f}=\frac{1}{\Delta \Omega_{f}} \int_{\Delta \Omega_{f}} \nabla f d \omega=\nabla \tilde{f}+\frac{1}{\Delta \Omega_{f}} \int_{\partial S_{w}} f \overrightarrow{d s}
$$

Results from using the $\Delta$ averaging theorem, see Whitaker [1999]. The fourth condition implies an unchanging porous medium morphology. All the above are well known from earlier work by Slattery [1980], Whittaker [1977] and Gray et al [1993]. At the same time, $\langle f\rangle_{f}$ and 
$\langle f\rangle_{s}$ fulfill neither the third Reynolds conditions nor all the consequences of the other Reynolds conditions.

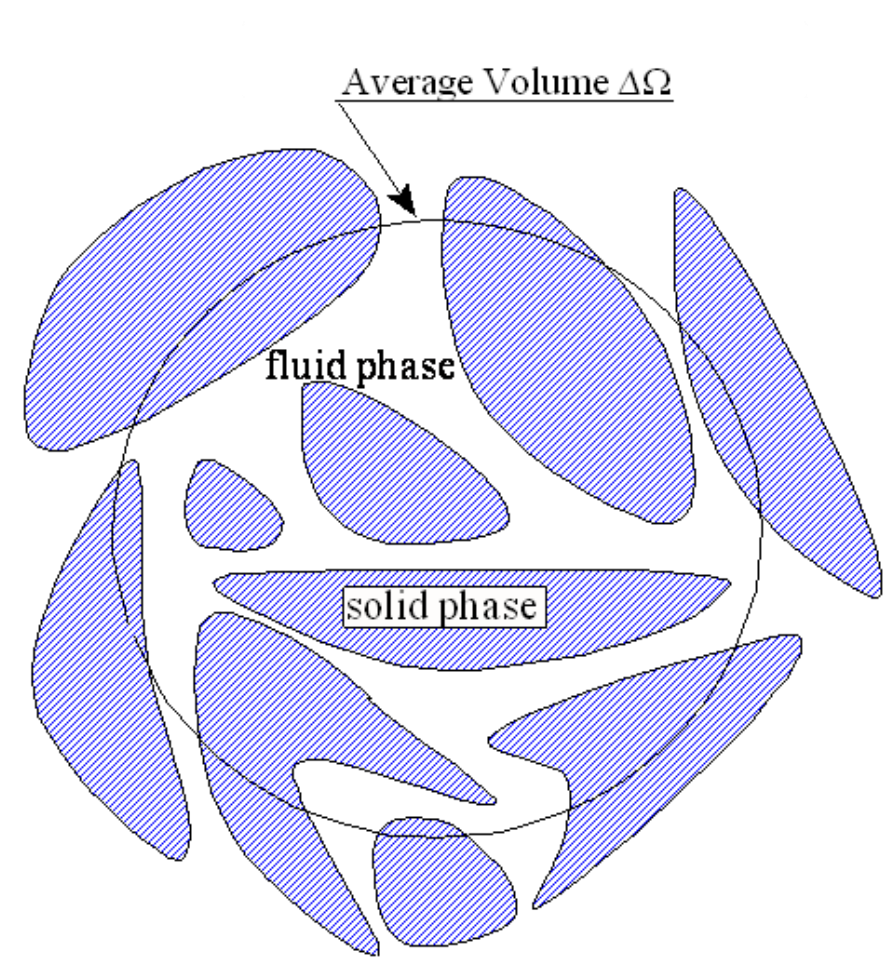

Figure 3: Representative Elementary Volume (REV)

Most of the following analysis is based on averaging techniques developed by Whitaker [1999] who focused on solving linear diffusion problems and by Travkin and Catton [1989] who focused on solving nonlinear turbulent diffusion problems. The divergent and non-divergent forms of the averaged convective term in the diffusion equation are

$$
\left.\left.<\nabla\left(T U_{i}\right)\right\rangle_{f}=\langle m\rangle \tilde{U}_{i} \frac{\partial}{\partial x_{i}} \tilde{T}+\nabla<\hat{T} \hat{u}_{i}\right\rangle_{f}+\frac{1}{\Delta \Omega} \int_{\partial S_{w}} T U_{i} \cdot \vec{d} s .-\tilde{T} \frac{1}{\Delta \Omega} \int_{\partial S_{w}} U_{i} \cdot \overrightarrow{d s}
$$

where $\tilde{T}=T-\hat{T}$. Applying the VAT rules and definitions to the heat flux (or mass flux) gradient term with spatially dependent heat conductivity $k_{T}$ yields 


$$
<\nabla \cdot\left(k_{T} \nabla T\right)>_{f}=\nabla \cdot\left(\langle m\rangle \tilde{k}_{T} \nabla \tilde{T}\right)+\nabla \cdot\left(\left\langle\hat{k}_{T} \nabla \hat{T}>_{f}\right)+\nabla \cdot\left[\tilde{k}_{T} \frac{1}{\Delta \Omega} \int_{\partial S_{w}} \hat{T} \overrightarrow{d s}\right]+\frac{1}{\Delta \Omega} \int_{\partial S_{w}} k_{T} \nabla T \cdot \vec{d} s,\right.
$$

Consistent application of these averaging rules and definitions to the conservation equations will allow the VAT governing equations to be systematically and rigorously derived.

\subsection{VAT Based Equations in Heterogeneous Media}

VAT based thermal physics and fluid mechanics governing equations in heterogeneous porous media developed from the Navier-Stokes equation and the thermal energy equation are the starting point and the basis for studying flow and heat transfer in porous media. The turbulent transport considered here is flow in a highly porous layer combined with a two energy equation model. For flow in porous media, the instantaneous velocity for the turbulent regime is represented by

$$
u=\bar{u}(x, z)+u^{\prime}(x, z, t)=\bar{u}_{k}(x, z)+\tilde{u}_{r}(x, z)+u_{r}^{\prime}(x, z)+u_{k}^{\prime}(x, z, t)
$$

where

$$
\begin{aligned}
\bar{u}(x, z) & =\frac{1}{t_{T}} \int_{t_{T}} u d t=\tilde{\bar{u}}(x, z)+u_{r}^{\prime}(x, z) \\
& =\bar{u}_{k}(x, z)+\tilde{u}_{r}(x, z)+u_{r}^{\prime}(x, z)
\end{aligned}
$$

$k$ signifies turbulence induced components that are independent of inhomogeneities in spatial dimensions and properties resulting from the multitude of porous medium channels (pores), and $r$ denotes the fluctuation contributions due to the porous medium inhomogeneity. The

fluctuation velocity $u_{r}^{\prime}(x, z)$ due to porous medium inhomogeneity is $u_{r}^{\prime}(x, z)=\hat{\bar{u}}(x, z)$. The temperature is represented by

$$
\begin{aligned}
T & =\bar{T}+T^{\prime}=\tilde{\bar{T}}(x, z)+T_{r}^{\prime}(x, z)+T^{\prime}(x, z, t) \\
& =\left\{\bar{T}_{k}(x, z)+\tilde{T}_{r}(x, z)+T_{r}^{\prime}(x, z)\right\}+T_{k}^{\prime}(x, z, t)
\end{aligned}
$$

\subsubsection{Development of Continuity Equation}


For incompressible flow, the continuity equation is

$$
\frac{\partial \bar{u}_{i}}{\partial x_{i}}=0
$$

Using Eq 12, the averaged continuity equation in the fluid phase for flow in a porous media is written

$$
\left\langle\frac{\partial \bar{u}_{i}}{\partial x_{i}}\right\rangle_{f}=\frac{\partial}{\partial x_{i}}\left(\left\langle\bar{u}_{i}\right\rangle_{f}\right)+\frac{1}{\Delta \Omega} \int_{\partial S_{w}}\left(\tilde{\bar{u}}_{i}+\hat{\bar{u}}_{i}\right) \cdot d \vec{s}=0
$$

\subsubsection{Development of Momentum Equation}

Starting from the momentum transport equation for incompressible flow

$$
\bar{u}_{j} \frac{\partial \bar{u}_{i}}{\partial x_{j}}=-\frac{1}{\rho_{f}} \frac{\partial \bar{p}}{\partial x_{i}}+\frac{\partial}{\partial x_{j}}\left(v \frac{\partial \bar{u}_{i}}{\partial x_{j}}-\overline{u_{i} u_{j}}\right)+F_{i},
$$

and using Eqs12, 13 and 14, the averaged momentum equation is written

$$
\begin{aligned}
& \langle m\rangle \tilde{\bar{u}}_{j} \frac{\partial \tilde{\bar{u}}_{i}}{\partial x_{j}}-\tilde{\bar{u}}_{i} \frac{1}{\Delta \Omega} \int_{\partial S_{w}}\left(\tilde{\bar{u}}_{j}+\hat{\bar{u}}_{j}\right) \cdot d \vec{s}+\frac{\partial}{\partial x_{j}}\left\langle\hat{\bar{u}}_{i} \hat{\bar{u}}_{j}\right\rangle_{f}+\frac{1}{\Delta \Omega} \int_{\partial S_{w}} \overline{u_{j}} \overline{u_{i}} \cdot d \vec{s} \\
& =-\frac{1}{\rho_{f}} \frac{\partial}{\partial x_{i}}(\langle m\rangle \tilde{\bar{p}})-\frac{1}{\rho_{f}} \frac{1}{\Delta \Omega} \int_{\partial S_{w}} \bar{p} d \vec{s}+\frac{\partial}{\partial x_{j}}\left(\langle m\rangle v \frac{\partial \tilde{\bar{u}}_{i}}{\partial x_{j}}\right)+\frac{1}{\Delta \Omega} \int_{\partial S_{w L}} \nu \frac{\partial \bar{u}_{i}}{\partial x_{j}} \cdot d \vec{s} \\
& +\frac{\partial}{\partial x_{j}}\left\langle-\overline{-u_{i}^{\prime} u_{j}^{\prime}}\right\rangle_{f}-\frac{1}{\Delta \Omega} \int_{\partial S_{w}} \overline{u_{i}^{\prime} u_{j}^{\prime}} d \vec{s}+\langle m\rangle \tilde{F}_{i}
\end{aligned}
$$

where $F_{i}$ incorporates the impact of micro-roughness and augmentation of the previous level of the simulation hierarchy. For many general flow situations, the Reynolds stress can be approximated by

$$
\overline{-u_{i}^{\prime} u_{j}^{\prime}}=v_{T}\left(\frac{\partial \bar{u}_{i}}{\partial x_{j}}+\frac{\partial \bar{u}_{j}}{\partial x_{i}}\right)
$$

For one-dimensional parallel flow, under steady-state conditions and no flow penetration through

$\partial S_{w}$, Eq. 21 has the form

$$
\begin{aligned}
& \frac{\partial}{\partial z}\left(\langle m\rangle\left(\tilde{v}_{T}+v\right) \frac{\partial \tilde{\bar{u}}}{\partial z}\right)+\frac{\partial}{\partial z}\left(\left\langle\tilde{v}_{T} \frac{\partial \tilde{\bar{u}}}{\partial z}\right\rangle_{f}\right)+\frac{\partial}{\partial z}\left(\langle-\hat{\bar{u}} \hat{\bar{w}}\rangle_{f}\right)+\frac{1}{\Delta \Omega} \int_{\partial S_{w T}} v_{T} \frac{\partial \bar{u}}{\partial x_{i}} \cdot d \vec{s} \\
& +\frac{1}{\Delta \Omega} \int_{\partial S_{w L}} v \frac{\partial \bar{u}}{\partial x_{i}} \cdot d \vec{s}-\frac{1}{\rho_{f} \Delta \Omega} \int_{\partial S_{w}} \bar{p} \vec{s}=\frac{1}{\rho_{f}} \frac{\partial\langle\bar{p}\rangle_{f}}{\partial x}
\end{aligned}
$$


An equation for one dimensional steady fully developed turbulent flow in a porous layer with regular packed bed structure characteristics and an impermeable interphase surface simplifies to

$$
\begin{aligned}
& \frac{\partial}{\partial z}\left(\langle m(z)\rangle\left(\tilde{v}_{T}+v\right) \frac{\partial \tilde{\bar{u}}(z)}{\partial z}\right)+\frac{1}{\Delta \Omega} \int_{\partial S_{w T}} v_{T} \frac{\partial \bar{u}}{\partial x_{i}} \cdot d \vec{s}+\frac{1}{\Delta \Omega} \int_{\partial S_{w L}} v \frac{\partial \bar{u}}{\partial x_{i}} \cdot d \vec{s}-\frac{1}{\rho_{f} \Delta \Omega} \int_{\partial S_{w}} \bar{p} d \vec{s} \\
& =\frac{1}{\rho_{f}} \frac{\partial(\langle m(z)\rangle \tilde{\bar{p}})}{\partial x}
\end{aligned}
$$

\subsubsection{Development of Turbulent Kinetic Energy Equation}

Following Rodi [1984], the turbulent kinetic is written

$$
\tilde{v}_{T}(z)\left(\frac{\partial \tilde{\bar{u}}}{\partial z}\right)^{2}+\frac{d}{d z}\left(\left(\frac{\tilde{v}_{T}}{\sigma_{b}}+v\right) \frac{d b(z)}{d z}\right)+\overline{X_{i}^{\prime} u_{i}^{\prime}}-\frac{g}{T \sigma_{b}}\left[\tilde{v}_{T} \frac{\partial \bar{T}}{\partial z}\right]+2 v\left(\frac{d b^{1 / 2}(z)}{d z}\right)^{2}=C_{D} C_{\mu} \frac{b^{2}(z)}{\tilde{v}_{T}}
$$

Where $C_{D}, C_{\mu}$ and $\sigma_{b}$ are empirical coefficients. $C_{D} C_{\mu} \approx 0.08$ and $\sigma_{b}=1$ appear to be reasonable values for the empirical constants. Assuming that most of the mean motion kinetic energy lost due to interaction of the flow with the porous medium solid obstacles translates into increasing the turbulent fluctuation energy, see [11], leads to the conclusion that

$$
\overline{X^{\prime} u^{\prime}}=c_{d} S_{w} \tilde{\bar{u}}^{3}
$$

It follows that the equation for the mean turbulent fluctuation energy $b(z)$ can be written in the form

$$
\tilde{v}_{T}(z)\left(\frac{\partial \tilde{\bar{u}}}{\partial z}\right)^{2}+\frac{d}{d z}\left(\left(\frac{\tilde{v}_{T}}{\sigma_{b}}+v\right) \frac{d b(z)}{d z}\right)+\frac{c_{d} S_{w}(z)}{\langle m\rangle} \tilde{\bar{u}}^{3}-\frac{g}{T \sigma_{b}}\left[\tilde{v}_{T} \frac{\partial \bar{T}}{\partial z}\right]+2 v\left(\frac{d b^{1 / 2}(z)}{d z}\right)^{2}=C_{D} C_{\mu} \frac{b^{2}(z)}{\tilde{v}_{T}}
$$

where the mean eddy viscosity $\tilde{v}_{T}$ can be determined using Prandtl's mixing length theory. By choosing $\sqrt{b}$ as the velocity scale, $\tilde{v}_{T}$ can be expressed as

$$
\tilde{v}_{T}=C_{\mu} \sqrt{b} L(z)
$$

where $L$ is the mixing length scale function defined by the assumed porous medium structure and

$$
b(z)=\frac{1}{2}\left(\overline{u^{2}}+\overline{v^{2}}+\overline{w^{\prime 2}}\right)
$$


is the turbulent kinetic energy. Eqs27 and 28 were used in the modeling of a regular porous medium filled channel transport by Travkin and Catton [1992, 1995] and Gratton et al.[1995, $1996]$.

\subsubsection{Development of Energy Equations}

To derive the volume averaged energy equation, the energy transport equation with turbulent transport,

$$
\rho_{f} c_{p f} \bar{u}_{j} \frac{\partial \bar{T}}{\partial x_{j}}=\frac{\partial}{\partial x_{j}}\left(k_{f} \frac{\partial \bar{T}}{\partial x_{j}}-\overline{T^{\prime} u_{j}^{\prime}}\right)+S_{T},
$$

is the starting point. Using Eqs. 12, 13 and 14, the averaged energy equation in fluid phase becomes

$$
\begin{aligned}
& \rho_{f} c_{p f}\left\{\langle m\rangle \tilde{\bar{u}}_{j} \frac{\partial \tilde{\bar{T}}}{\partial x_{j}}-\tilde{\bar{T}} \frac{1}{\Delta \Omega} \int_{\partial S_{w}}\left(\tilde{\bar{u}}_{j}+\hat{\bar{u}}_{j}\right) \cdot d \vec{s}\right\}+\rho_{f} c_{p f}\left\{\frac{\partial}{\partial x_{j}}\left\langle\hat{\bar{T}}_{\hat{\bar{u}}}\right\rangle_{f}+\frac{1}{\Delta \Omega} \int_{\partial S_{w}} \overline{u_{j}} \bar{T} \cdot d \vec{s}\right\} \\
& =\frac{\partial}{\partial x_{j}}\left(\langle m\rangle k_{f} \frac{\partial \tilde{\bar{T}}}{\partial x_{j}}\right)+\frac{1}{\Delta \Omega} \int_{\partial S_{w L}} k_{f} \frac{\partial \bar{T}}{\partial x_{j}} \cdot d \vec{s}+\frac{\partial}{\partial x_{j}}\left(\frac{k_{f}}{\Delta \Omega} \int_{\partial S_{w}} \hat{\bar{T}} d \vec{s}\right) \\
& +\frac{\partial}{\partial x_{j}}\left\langle-\overline{T^{\prime} u_{j}^{\prime}}\right\rangle_{f}-\frac{1}{\Delta \Omega} \int_{\partial S_{w}} \overline{T^{\prime} u_{j}^{\prime}} d \vec{s}+\langle m\rangle \tilde{S}_{T}
\end{aligned}
$$

The term $S_{T}$ represents a heat source. By analogy with the concept of eddy viscosity, the turbulent fluctuation terms are proportional to the mean temperature gradients. For two dimensional heat transfer in a channel and no flow penetration through $\partial S_{w}$, the above equation simplifies to

$$
\begin{aligned}
& c_{p f} \rho_{f}\langle m\rangle \tilde{\bar{u}}(z) \frac{\partial \tilde{\bar{T}}(x, z)}{\partial x}=\langle m\rangle \frac{\partial}{\partial x_{i}}\left(\left(\tilde{k}_{T}+k_{f}\right) \frac{\partial \tilde{\bar{T}}(x, z)}{\partial x_{i}}\right) \\
& +\frac{1}{\Delta \Omega} \int_{\partial S_{w T}} k_{T} \frac{\partial \bar{T}}{\partial x_{i}} \cdot d \vec{s}+\frac{1}{\Delta \Omega} \int_{\partial S_{w L}} k_{f} \frac{\partial \bar{T}}{\partial x_{i}} \cdot d \vec{s}
\end{aligned}
$$

where $\tilde{k}_{T}$ is the turbulent heat conductivity. The solid phase energy equation is 


$$
\frac{\partial}{\partial x_{i}}\left(k_{s}(x, z) \frac{\partial T_{s}(x, z)}{\partial x_{i}}\right)=0
$$

where $k_{s}$ is the effective heat conductivity in the solid phase. For two dimensional heat transfer in the solid phase, the volume averaged solid phase thermal energy equation is written

$$
\begin{aligned}
& \frac{\partial}{\partial x}\left((1-\langle m\rangle) \tilde{k}_{s T}(x, z) \frac{\partial T_{s}(x, z)}{\partial x}\right)+\frac{\partial}{\partial z}\left((1-\langle m\rangle) \tilde{k}_{s T}(x, z) \frac{\partial T_{s}(x, z)}{\partial z}\right) \\
& +\frac{1}{\Delta \Omega} \int_{\partial S_{w T}} k_{s T} \frac{\partial T_{s}}{\partial x_{i}} \cdot d \vec{s}=0
\end{aligned}
$$

where $d \vec{s}_{1}=-d \vec{s}$.

\subsection{Closure of the VAT Equations}

To make use of the VAT based equations, four terms are unknown and needed for closure. These terms are averaged for each REV, and are $S_{w}$, the surface area of the fluid to solid interface, and $\langle\mathrm{m}\rangle$, the porosity, or the area of the fluid divided by the total area. These two terms can be found from the geometry of the heat exchanger, so they are no problem to obtain. The other two terms needed is the thermal and energy characteristics of the REV, namely the heat transfer and drag coefficients. These can be obtained in several methods, such as experiment or CFD, as explained below.

Closure theories for transport equations in heterogeneous media have been the primary measure of advancement and for measuring success in research on transport in porous media. It is believed that the only way to achieve substantial gains is to maintain the connection between porous medium morphology and the rigorous formulation of mathematical equations for transport. There are only two well-known types of porous media morphologies for which researchers have had major successes; namely straight parallel pores and equal size spherical 
inclusions. But even for these morphologies, not enough evidence is available to state that the closure problems for them "are fully closed". 


\section{Methods of Obtaining Closure}

Before moving to development of the closure relationships, it is interesting to note that using a particular length scale leads to a parameter that is very beneficial when scaling heat transfer and friction factor results. It was shown by Travkin and Catton [2001] that media of globular morphologies can be described in terms of $S_{w},\langle m\rangle$ and $d_{p}$ and can generally be considered to be spherical particles with

$$
S_{w}=\frac{6(1-<m>)}{d_{p}}, \quad d_{h}=\frac{2}{3} \frac{<m>}{(1-<m>)} d_{p}
$$

This expression has the same dependency on equivalent pore diameter as found for a one diameter capillary morphology leading naturally to

$$
S_{w}=\frac{6(1-<m>)}{d_{p}}=\frac{6(1-<m>)}{\left(\frac{3}{2} \frac{(1-<m>)}{<m>} d_{h}\right)}=\frac{4<m>}{d_{h}}
$$

This observation leads to defining a simple "universal" porous media scale

$$
d_{h}=\frac{4\langle m\rangle}{S_{w}}
$$

that meets the needs of both morphologies; capillary and globular. This was also recognized by Whitaker [1972] when he used a very similar (differing by a constant) length scale when correlating heat transfer for a wide variety of morphologies. Here we will show results for correlation of friction factor and heat transfer coefficient for a fin tube heat exchanger. The closure terms in the VAT momentum equations are, see Eq. (24), 


$$
\begin{aligned}
\frac{1}{\Delta \Omega} \int_{\partial S_{w L}} v & \frac{\partial \bar{u}_{i}}{\partial x_{j}} \cdot d \vec{s}+\frac{1}{\Delta \Omega} \int_{\partial S_{w}} v_{T} \frac{\partial \bar{u}_{i}}{\partial x_{j}} d \vec{s} \\
& -\frac{\partial}{\partial x_{j}}\left\langle\hat{\bar{u}}_{i} \hat{\bar{u}}_{j}\right\rangle_{f}-\frac{1}{\rho_{f}} \frac{1}{\Delta \Omega} \int_{\partial S_{w}} \bar{p} d \vec{s}
\end{aligned}
$$

The first and second terms are the laminar and turbulent flow contributions to skin friction, the third term represents the spatial flow oscillations induced by the porous media morphology and the fourth term is the form drag.

A natural way to close the integral terms in transport equations is to integrate over the inter-phase surface, or of some other outlined areas of this surface. From a physical point of view, the integration terms in Eq.(38) represent momentum loss due to the friction resistance and pressure (form) drag over all inter-phase surfaces. It should be noted that this is relatively easy with modern commercial CFD packages.

The pressure drag resistance integral terms appearing in Eq. (38) are closed in a manner similar to that for a pressure resistance coefficient over an obstacle or hydrofoil, where according to its definition the form drag is defined as

$$
c_{d p}=2 \frac{\int_{\partial S_{w}} \bar{p} \mathrm{~d} \vec{s}}{\left(\rho_{f} \tilde{\bar{u}}^{2} S_{w p}\right)}
$$

where $S_{w p}$ is the cross flow projected area. Now, using this definition, the pressure drag resistance integral term can be closed with

$$
\frac{1}{\rho_{f} \Delta \Omega} \int_{\partial S_{w}} \bar{p} \mathrm{~d} \vec{s}=\frac{1}{2} c_{d p} S_{w p} \tilde{\tilde{u}}^{2}
$$

Similarly, the skin friction resistance integral terms appearing in Eq. (24) are closed using the definition of skin friction coefficient defined by 


$$
c_{f}=2 \frac{\int_{\partial S_{w}} \tau_{w} \mathrm{~d} \vec{s}}{\left(\rho_{f} \tilde{\bar{u}}^{2} S_{w}\right)}
$$

Travkin and Catton (1995) showed that using the proper length scale, like the one represented by Eq. (37), enables one to write the friction factor of porous media in the following form

$$
f_{f}=\frac{A}{\operatorname{Re}_{h}}+B
$$

where $f_{f}$ is the Fanning friction factor and the Reynolds number is defined as

$$
\operatorname{Re}_{h}=\frac{\tilde{\bar{u}} \cdot d_{h}}{v}
$$

with $A=100 / 3$ and $B=7 / 12$ for the Ergun equation for packed bed porous media. These constant values were shown to be very close to the correlation developed by Klevon and Matros (1990) with $\mathrm{A}=36.3$ and $\mathrm{B}=0.45$ for spherical particles and $\mathrm{A}=37.6$ and $\mathrm{B}=0.585$ for cylindrical particles. Travkin and Catton (1995) were able to show that by choosing the above form, it allows one to collapse true capillary flow and flow in a bed of spheres. This is a significant accomplishment, since it spans the physical description from globular to capillary geometry with a single length scale.

The friction factor presented by Eq. (42) has two distinctive terms, each corresponding to different pressure drop mechanisms. One is attributed to the viscous flow, that is linearly proportional to velocity and the other term is due to convection of the fluid momentum that is proportional to the velocity squared. Furthermore, Travkin and Catton (1995) stated that friction factor is related to a closure for VAT momentum equations and they showed that

$$
c_{d} \cong f_{f}
$$


It was shown that the closure equation Eq. (38) is an exact definition of friction factor. Note that for fully developed flow Eq. (42) is more strictly defined as

$$
c_{d}=f_{f}=\frac{A}{\operatorname{Re}_{h}}+B
$$

where $\mathrm{A}$ and $\mathrm{B}$ are constants corresponding to the different types of morphology of porous media.

The fluid energy equation can be closed in various ways and in general will depend on how many of the integrals appearing in the VAT equation one uses and lumps into a single transport coefficient, see Travkin and Catton (2001). The nature of the equation shows that the energy transferred from the surface is integrated over an area and then divided by a REV volume; therefore, the heat transfer coefficient is defined in terms of porous media morphology, usually described by specific surface and porosity. The simplest, most intuitive and most often used variation includes only the intra-phase heat transfer and is defined as

$$
h_{1}=\frac{\frac{1}{\Delta \Omega} \int_{\partial S_{w}}\left(k_{f}+k_{T}\right) \nabla T_{f} d \mathbf{S}}{S_{w}\left(\tilde{T}_{s}-\tilde{T}_{f}\right)}
$$

By the same token one could include more terms, such as spatial velocity and temperature fluctuations yielding a new form defined by

$$
h_{2}=\frac{\frac{1}{\Delta \Omega} \int_{\partial S_{w}}\left(k_{f}+k_{T}\right) \nabla T_{f} d \mathbf{S}-\rho_{f} c_{p f} \nabla \cdot\left(\langle m\rangle \hat{\mathbf{u}}_{f} \hat{T}_{f}\right)}{S_{w}\left(\tilde{T}_{s}-\tilde{T}_{f}\right)}
$$

Lastly, a third form could be defined that uses all of the fluid energy equation integral terms to yield the most complete form,

$$
h_{3}=h_{1}-\frac{\rho_{f} c_{p f} \nabla \cdot\left(\langle m\rangle \hat{\mathbf{u}}_{f} \hat{T}_{f}\right)}{S_{w}\left(\tilde{T}_{s}-\tilde{T}_{f}\right)}+\frac{\nabla \cdot\left[\frac{k_{f}}{\Delta \Omega} \int_{\partial S_{w}} T_{f} d \mathbf{S}\right]}{S_{w}\left(\tilde{T}_{s}-\tilde{T}_{f}\right)}
$$


The number and the nature of the closure terms in the macro scale equations were rigorously derived from micro scale governing equations and they are clearly defined. The way one defines a heat transfer coefficient can vary as shown by the three definitions of the heat transfer coefficient and therefore, one has to be careful on which variation is applicable to the problem that one tries to solve. When in doubt, one should use the complete form given by Eq. (48). In most engineered devices, the geometry is regular and a well chosen REV will lead to only the first type being needed. The first and third terms in Eq. (48) can be estimated using scaling arguments. It is assumed that the length scale within the REV is $l$ and that the whole REV length scale is $L$. With these assumptions, the first term can be estimated as

$$
\frac{k_{f}}{\Delta \Omega} \int_{\partial S_{w}} \nabla T_{f} d \mathbf{S}=O\left(\frac{k_{f} \cdot \Delta T_{f}}{L \cdot l}\right)
$$

while the third term is scaled as

$$
\nabla \cdot\left[\frac{k_{f}}{\Delta \Omega} \int_{\partial S_{w}} T_{f} d \mathbf{S}\right]=O\left(\frac{k_{f} \cdot \Delta T_{f}}{L^{2}}\right)
$$

Since the macro length scale is much larger that the micro scale, $L>l$, the third term is much smaller than the first and can therefore be ignored yielding

$$
h_{3} \cong h_{2}=\frac{\frac{k_{f}}{\Delta \Omega} \int_{\partial S_{w}} \nabla T_{f} d \mathbf{S}-\rho_{f} c_{p f} \nabla \cdot\left(\langle m\rangle \hat{\mathbf{u}}_{f} \hat{T}_{f}\right)}{S_{w}\left(\tilde{T}_{s}-\tilde{T}_{f}\right)}
$$

For conditions of fully developed flow, the spatial flow oscillations within the REV are constant or nearly constant, generally depending on the boundary conditions; either constant flux 
or iso-thermal. Thus, the product of flow oscillations is also constant and the second term in Eq. (47) or Eq.(48) is essentially zero, yielding

$$
h_{2} \cong h_{1}=\frac{\frac{k_{f}}{\Delta \Omega} \int_{\partial S_{w}} \nabla T_{f} d \mathbf{S}}{S_{w}\left(\tilde{T}_{s}-\tilde{T}_{f}\right)}
$$

This is an important result as it greatly simplifies the scope of the problem. The use of scaling arguments showed that for fully developed flow, small scale heat transfer is the dominating term in the closure formulation and it is much greater than the heat transfer on the larger macro scale. Furthermore, for spatially periodic media morphology, the velocity and temperatures fluctuation term is equal to zero, which further states that the main mechanism of intra-phase transport is the small scale intra-phase heat transfer defined by the energy equation closure (Eq.50)).

How the REV is selected is very important. The selection for a finned tube exchanger, Fig. 1, is seen to repeat in both the cross-stream and flow directions. The fluctuation terms in Eq 51 were evaluated and found to be zero. This, not unexpected, result simplifies the CFD task.

\subsection{Closure Using Experimental Results}

An example of how closure is obtained from experimental data is given by Zhou, Hansen, and Catton [2010]. The porosity and specific surface are geometrically defined terms. Figure 2 depicts the fin geometry studied in their work. For a plate finned tube heat exchanger, the porosity for the air side is

$$
<m_{1}>=1-\frac{\delta_{f}}{F_{p}}-\frac{\pi D_{c}^{2}\left(F_{p}-\delta_{f}\right)}{4 P_{l} P_{t} F_{p}}
$$

and for the water side is 


$$
<m_{2}>=\frac{\pi D_{c}^{2}}{4 P_{l} P_{t}}
$$

The specific surface area for the air side is given by

$$
S_{w 1}=\frac{2 P_{l} P_{t}-2 \pi\left(\frac{D_{c}}{2}\right)^{2}+\pi D_{c}\left(F_{p}-\delta_{f}\right)}{P_{l} P_{t} F_{p}}
$$

and for the water side

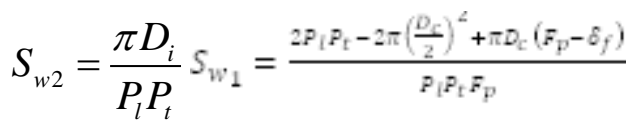

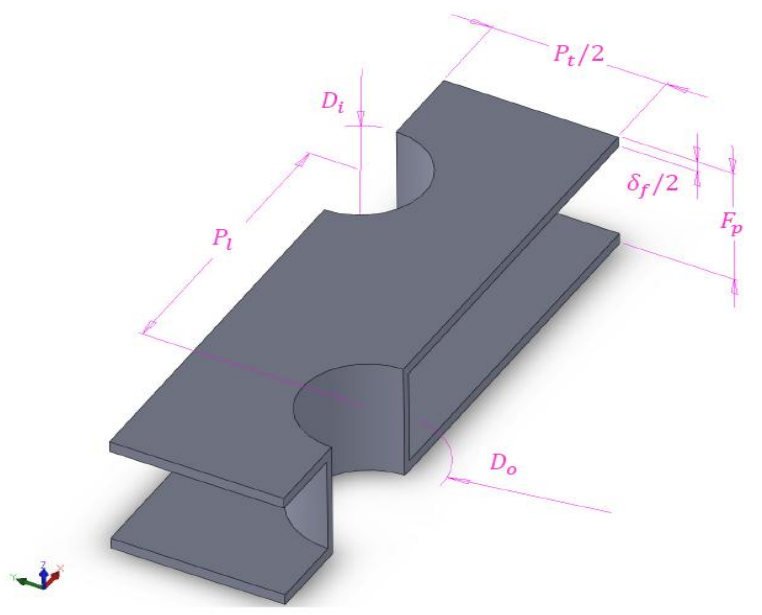

Figure 4: REV for a Finned Tube Heat Exchanger

Wang (2000) developed precise correlations for the friction factor for the air side of plain finned tube heat exchangers. They were scaled with the fin collar outside diameter and the maximum velocity. The friction factor results are shown on the left of Fig. 3 and the rescaled results are shown on the right clearly demonstrating the value of the VAT based length scale. Wang's (2000) experimental data were rescaled using the length scale given by Eq. (37). The results were surprisingly good, collapsing all the data onto a single curve. This in turn, with the help of JMP 8, an available statistical analysis tool, enabled us to develop a simple correlation for the friction factor, 


$$
c_{d 1}=f=\frac{13.9}{\operatorname{Re}_{d_{h}}}+0.015
$$

A comparison of some values of A and B with other morphologies is shown in Table 1. The type of correlation given by Eq. (40) is very powerful allowing one to estimate the result when no data are available.

The VAT closure for the fluid energy equation is found in terms of the heat transfer coefficient $h$, see Eq. (48). Experimental data from Xie (2009) for $\mathrm{N}=7$ and by Tang (2009) for $\mathrm{N}=9$ and $\mathrm{N}=12$ were plotted on a single graph for each of the length scales, $D_{c}$ and $d_{h}$, and are shown in Fig. 4. It can be seen that all the data collapse very close to a single curve when scaled using the VAT based length scale given by Eq. (37). Using JMP 8, a correlation of the rescaled data was found and is

$$
N u_{d_{h}}=\frac{h_{1} d_{h_{1}}}{k_{1}}=0.061 \operatorname{Re}_{d_{h}}^{0.72} \operatorname{Pr}^{1 / 3}
$$

It is worth noting that the correlation is similar to the Dittus-Boelter correlation $\left(N u=0.023 \operatorname{Re}_{d_{h}}^{0.8} \operatorname{Pr}^{1 / 3}\right.$ ) for forced convection and in some sense a testament to the power of the hydraulic diameter approach to scaling. 

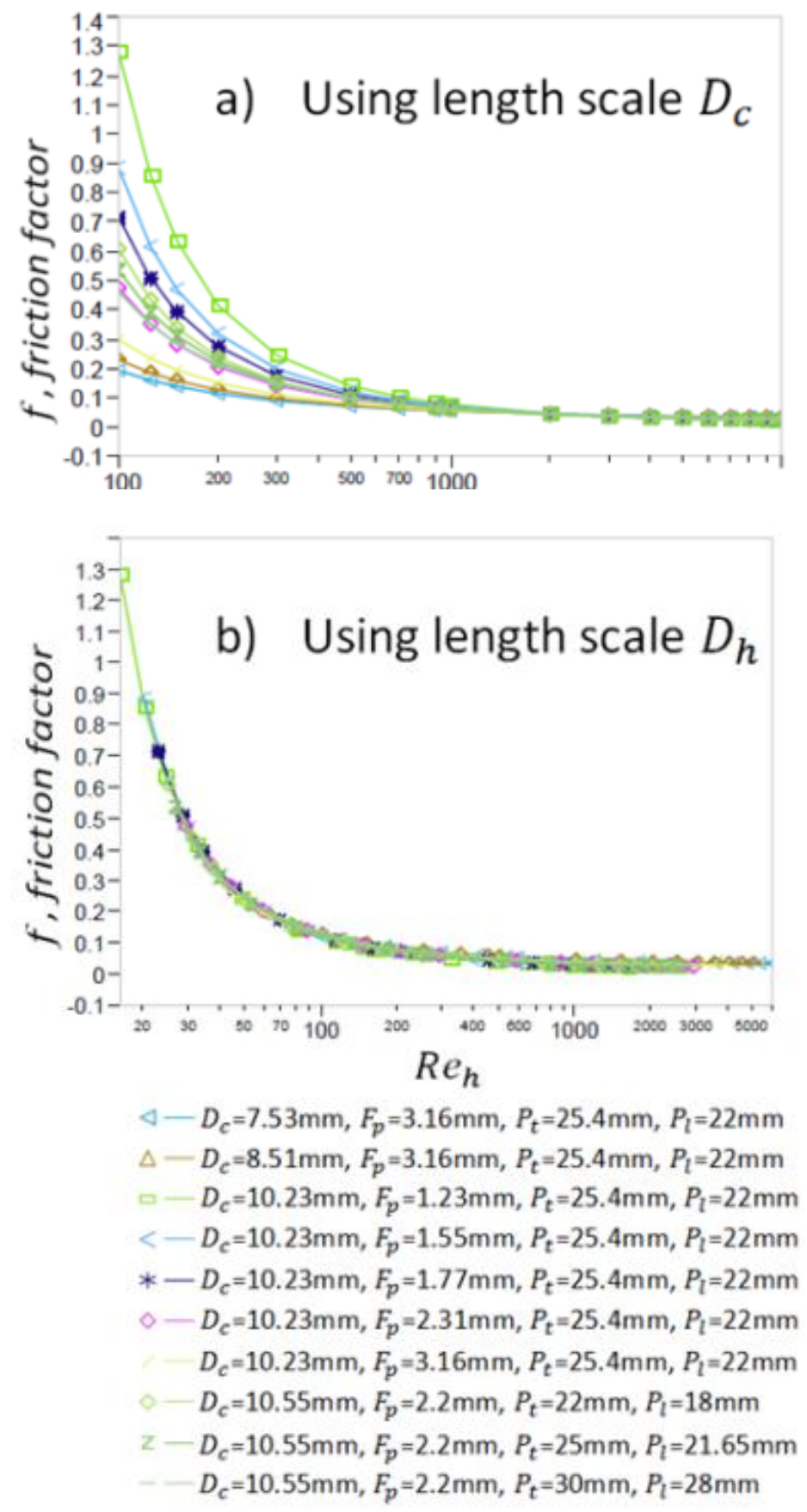

Figure 5: Overlay plot of all the data using length scales $D_{c}$ and $D_{h}$

\subsection{Closure Using CFD Results}

Numerical calculations were performed by Vadnjal (2009) at different Reynolds numbers for a simple body center cubic packing arrangement of spheres, see Figure 7. This configuration 
was chosen because it has been well studied, is not too complex and, although not always in agreement, a great deal of data exists.

\begin{tabular}{|c|c|c|c|}
\hline & A & B & Porosity range \\
\hline Packed bed & $100 / 3$ & $7 / 12$ & $0.3-0.72$ \\
\hline Pin fins-inline & 50 & 0.145 & $0.65-0.91$ \\
\hline Pin fins-staggered & 50 & 0.145 & $0.65-0.91$ \\
\hline $\begin{array}{c}\text { Plain finned tube- } \\
\text { staggered }\end{array}$ & 13.9 & 0.0153 & $0.65-0.9$ \\
\hline
\end{tabular}

Table 1: Closure coefficients for different porous media morphology

Once the CFD results were obtained, the integrations needed to evaluate the terms in Eq. (38) were used to obtain drag resistance coefficients (friction factor) and compared to the well established results of Ergun [1952], Morcom [1946], Burke and Plumer [1928] and a correlation developed by Ergun [1952]. The calculated value included all three terms given by Eq. (38) including the fluctuating terms. The experimental data is representative of porous media morphology composed of different size spheres, sand and pulverized coke.

The comparison is shown in Figure 6 and the results show the validity of the VAT calculations for laminar flow regimes where the Reynolds number, $R e_{h}$, ranged from 0.4 to 124 . Agreement is good for Reynolds numbers up to approximately 50, whereas for higher values, the calculation yielded slightly higher values of the friction factor. It is interesting to note that the curve starts to change at the particular Reynolds number where the flow is transitioning from a laminar to a turbulent regime. The purpose of this work was to model laminar flow and to avoid CFD turbulence modeling and the approximations that are associated with it. 

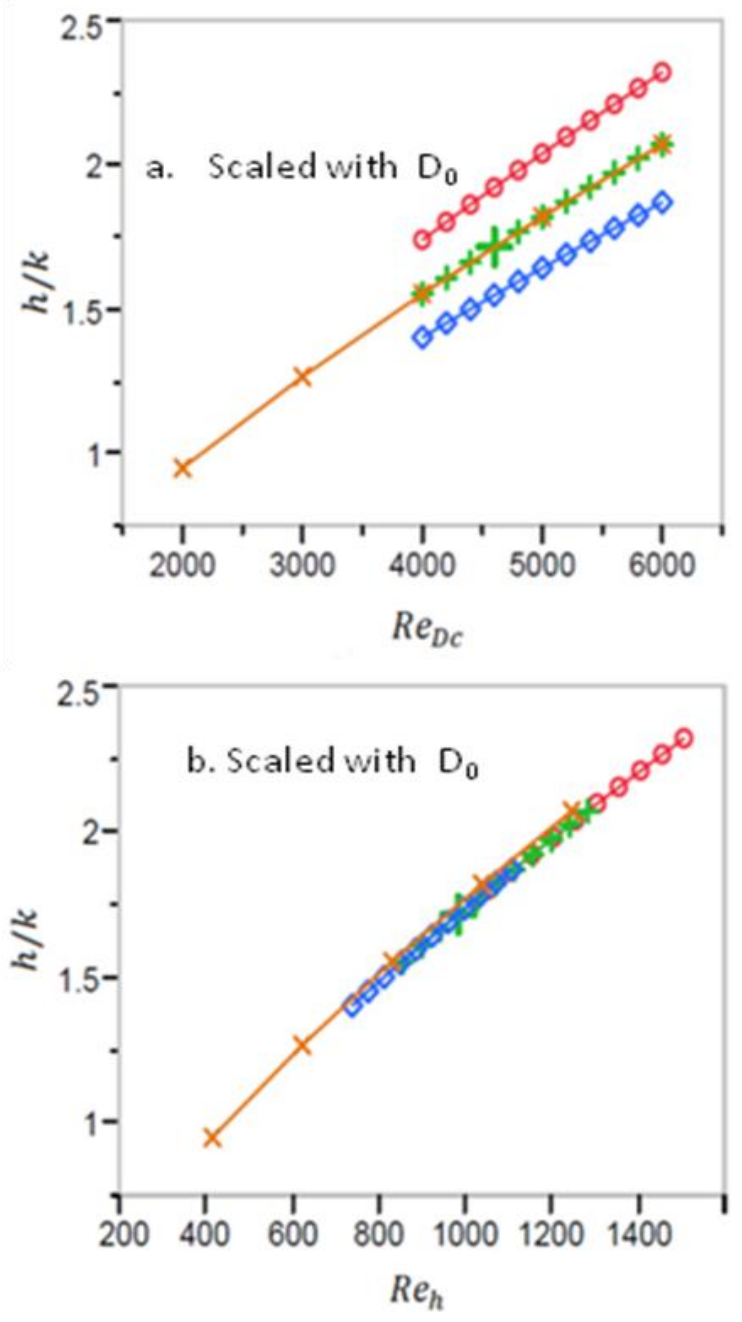

$$
\begin{aligned}
& O-D_{o}=16 \mathrm{~mm}, N=8, \text { Tang (2009) } \\
& +-D_{o}=18 \mathrm{~mm}, N=9 \text {, Tang (2009) } \\
& \diamond-D_{o}=20 \mathrm{~mm}, N=12 \text {, Tang (2009) } \\
& \times-D_{o}=18 \mathrm{~mm}, N=7 \text {, Xie (2009) }
\end{aligned}
$$

Figure 6: Comparison of heat transfer coefficients using $D_{c}$ and $D_{h}$ as the length scales. 


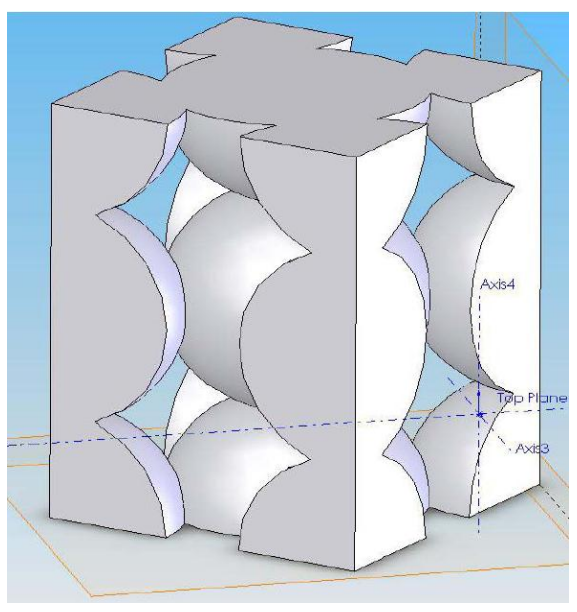

\section{Figure 7: Computational domain morphology}

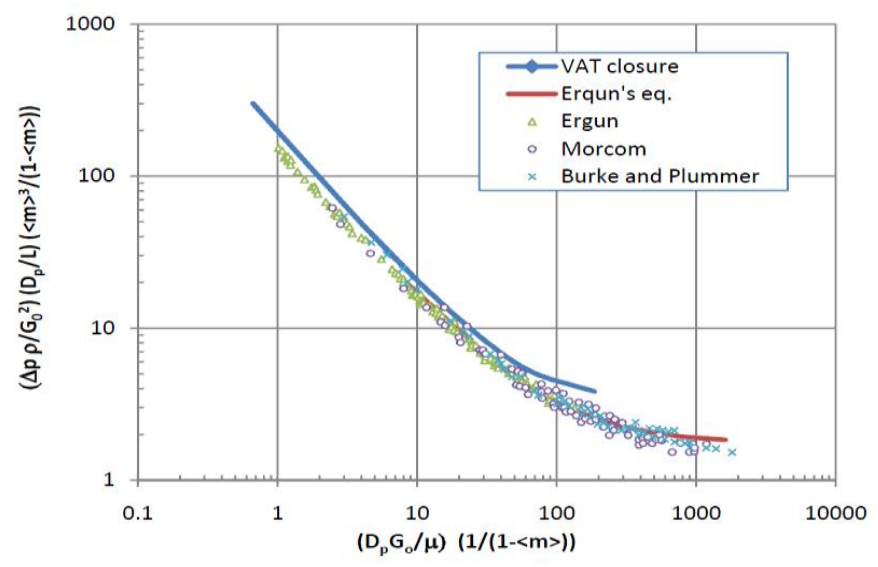

\section{Figure 8: Comparison of VAT closure term $c_{d}$ by CFD to data}

Closure for the momentum equation shows that the drag resistance coefficient has two distinctive contributions: one is due to pressure and the other is due to viscous forces. The relative contributions to the total drag resistance coefficient are shown in Figure 9, where it can be seen higher Reynolds numbers lead to a pressure contribution that is much greater than the viscous contribution. This demonstrates the need to describe the morphology in terms of both the total specific area for calculation of viscous forces, and the specific area normal to the flow direction to determine the form drag. Further, the form of the drag coefficient lends itself to a single porous media type code for a wide variety of internal structures. 


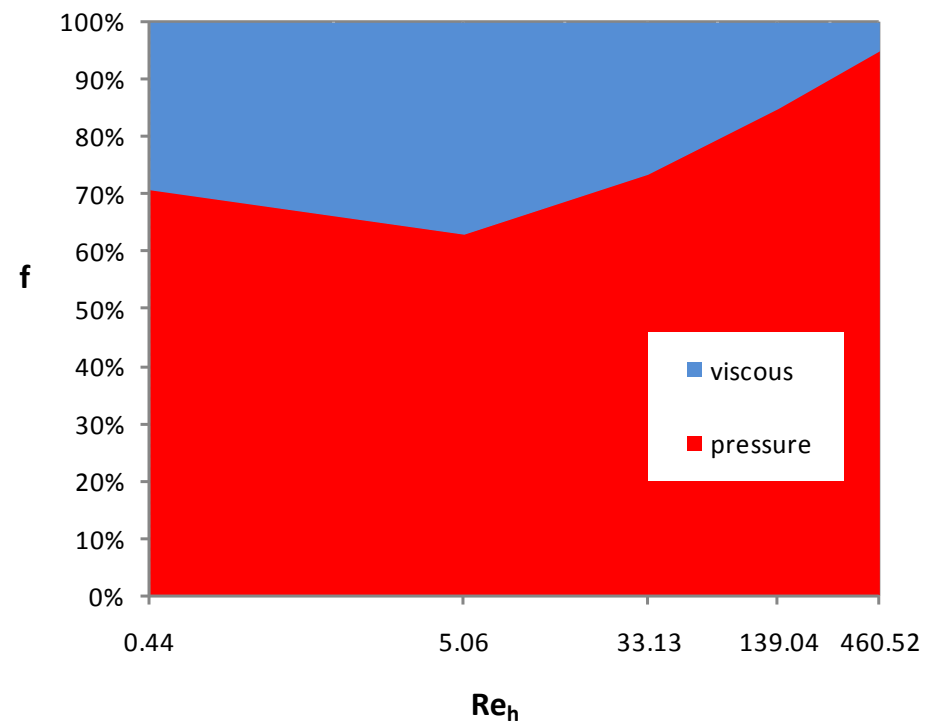

Figure 9: Contribution of viscous and pressure forces to the drag resistance coefficient 
To obtain energy equation closure, the heat transfer coefficient is obtained by resolving the fluid flow and the heat transfer on the lower micro scale. The use of scaling arguments showed that for fully developed flow, small scale heat transfer is the dominating term in the closure formulation and it is much greater than the heat transfer on the larger macro scale. Furthermore, for spatially periodic media morphology, the velocity and temperatures fluctuation term is equal to zero, which further states that the main mechanism of intra-phase transport is the small scale intra-phase heat transfer defined by the energy equation closure (Eq.(52)).

The numerical results for heat transfer closure are shown in Figure 10 and are compared to the various experimental results presented by the correlation given by Whitaker [1972]. The result obtained by VAT closure included only the first term and show fair agreement with the data, therefore one can confirm that the heat transfer coefficient when scaled correctly can be computed numerically with satisfactory results.

The Local and average Nusselt number shown in Figure 10, differed from the average as expected and therefore one has to pay special attention when using heat transfer correlations and especially when one uses the heat transfer coefficient with the VAT governing equations to solve large scale problems. When compared to the available experimental data, one can conclude that calculated values, either local or average heat transfer coefficients, are within the experimental bounds. However it is not so clear how the experimental data was processed, whether the values are local or averaged and therefore the differences are obvious, and even a couple of orders apart when Reynolds number is small. 


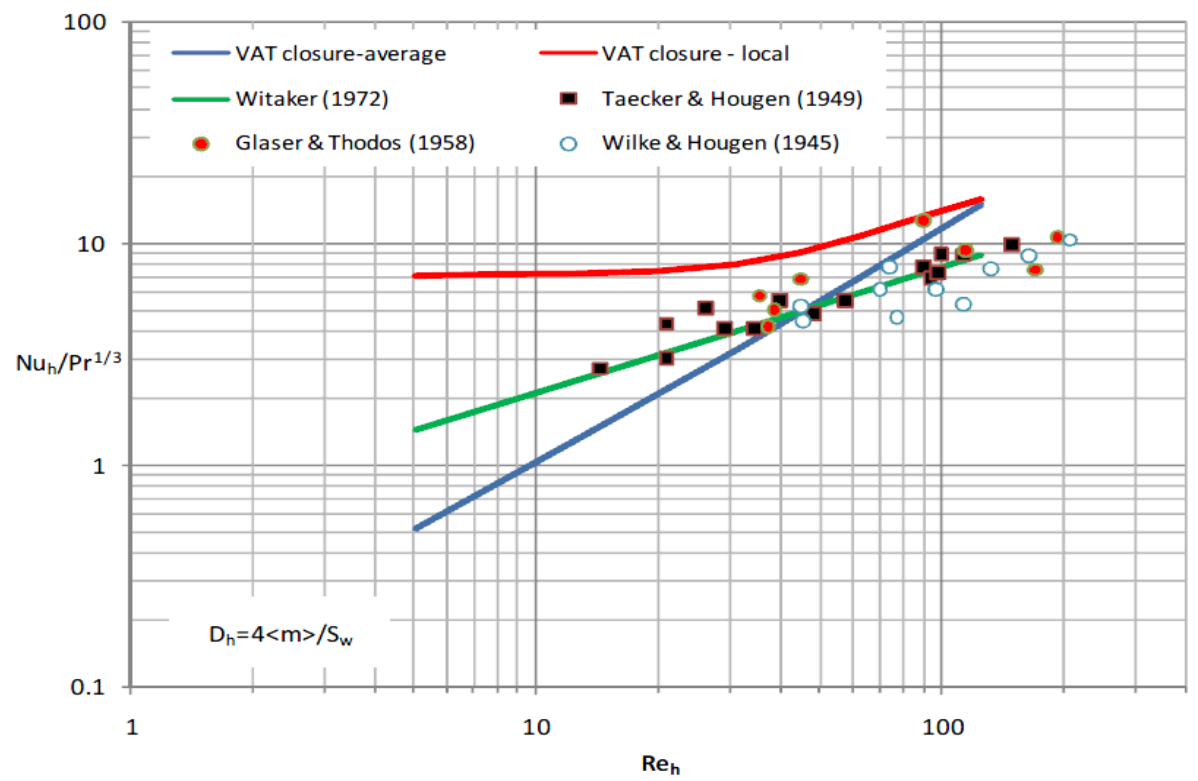

Figure 10: Comparison of VAT closure Nu obtained by CFD with experiment

Having obtained solutions at the micro scale, the entrance effects in a porous media were addressed. The interest here was to determine how averaged temperatures and heat transfer coefficients vary as the flow is developing from its initial uniform entrance condition to its thermally fully developed state. The micro scale solution of a 5 REV long morphology was averaged over a single REV. Average temperatures for fluid and solid phase were computed for a flow with $\mathrm{Re}_{\mathrm{h}}=31.2$ at different locations and the results are shown on Figure 11. As expected, the average temperature of fluid and solid are increasing from the inlet to the exit. The largest temperature difference is at the inlet while the smallest is at the exit. Intuitively, one would to expect the heat transfer coefficient to be largest at the entrance and that it would gradually asymptote to some value until thermally fully developed flow is reached.

By inspecting the heat transfer coefficient definition, Eq.(52), one can see that it has two parts; one in the numerator and the one in the denominator, each representing the physics of interfacial heat transfer. The integral term represents the heat transfer across the interface while 
the temperature difference represents its potential. Error! Reference source not found. shows their values and their development from the inlet to the exit and it is interesting to see that their slope is almost identical. With both numerator and denominator computed, the local heat transfer coefficient can be computed and the results are plotted on Figure 12. It is surprising to see that the local coefficient is almost constant throughout the domain and it is not largest at the inlet where the temperature difference between solid and fluid is the largest. Those results were achieved at fairly low Reynolds number and to make this result more convincing, the same steps were repeated at a higher Reynolds number, $\operatorname{Re}_{h}=440.8$ and are shown on Figure 13 and Figure 14. Again it is shown that the local heat transfer coefficient is essentially constant from the inlet to the exit.

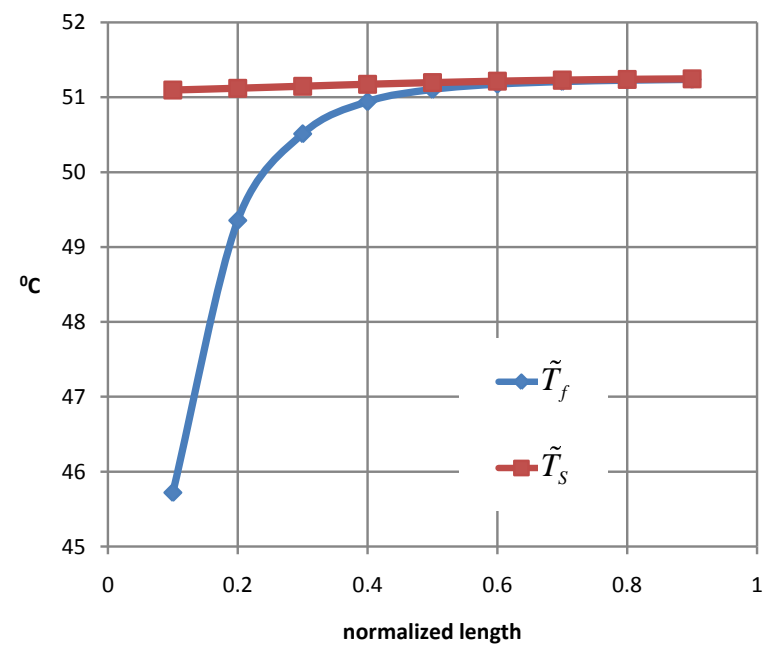

Figure 11: Development of averaged temperatures of fluid and solid phase at $\operatorname{Re}_{\mathrm{h}}=31.2$ 


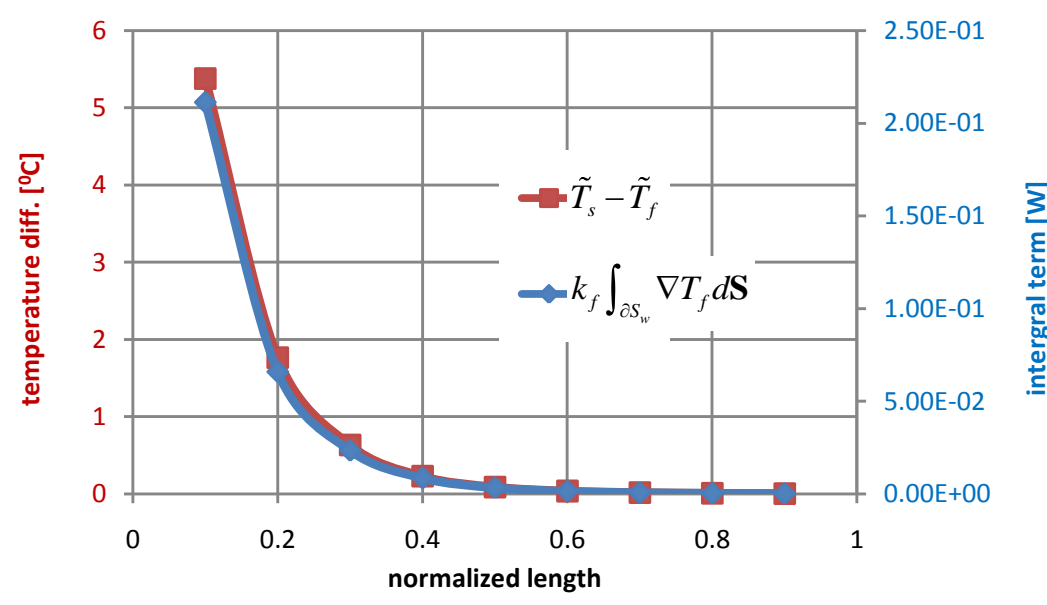

Figure 12: Temperature Differences at $\operatorname{Re}_{\mathrm{h}}=31.2$

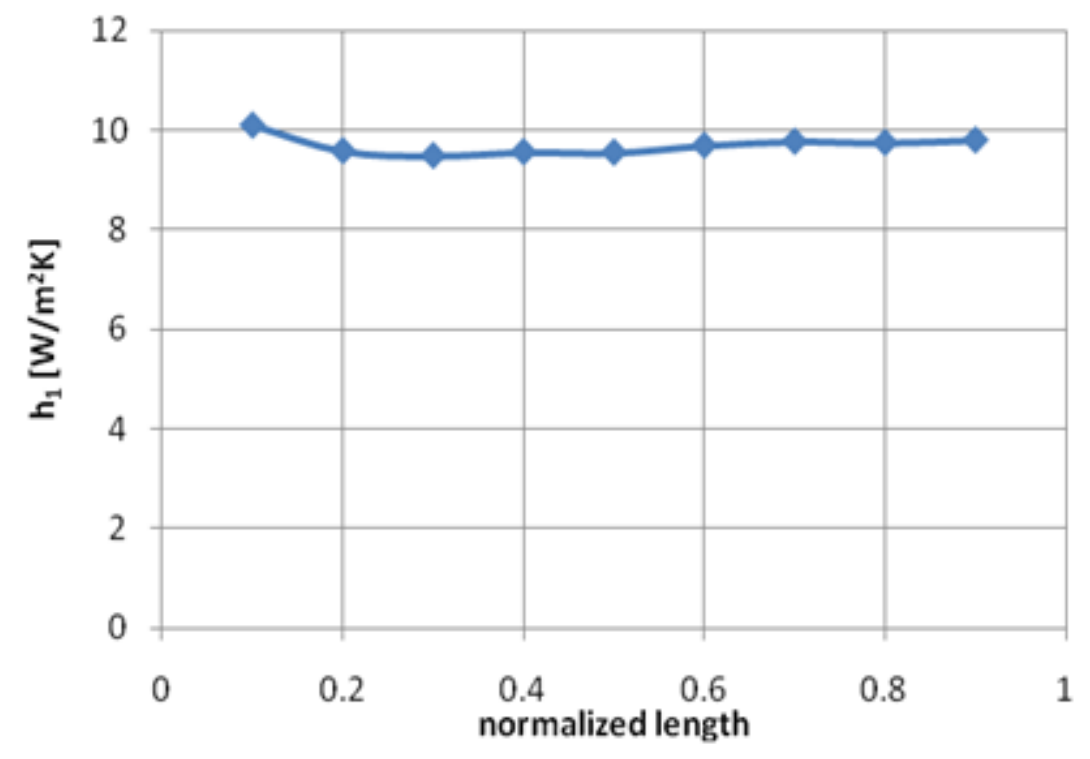

Figure 13: Local Heat Transfer Coeficient at $\operatorname{Re}_{\mathrm{h}}=31.2$

In general, at the entrance, the point local and average heat transfer coefficient will both be some large number and they both asymptote to corresponding numbers as the Graetz number goes to a large value. For the micro scale solution obtained for the whole domain, a local point value heat transfer coefficient on a solid-fluid interface was found to be largest at the entrance 
and gradually decreases as flow progresses further downstream. However, the local VAT average heat transfer is essentially independent of entrance region. This remarkable result is very helpful when developing a simple model based on VAT.

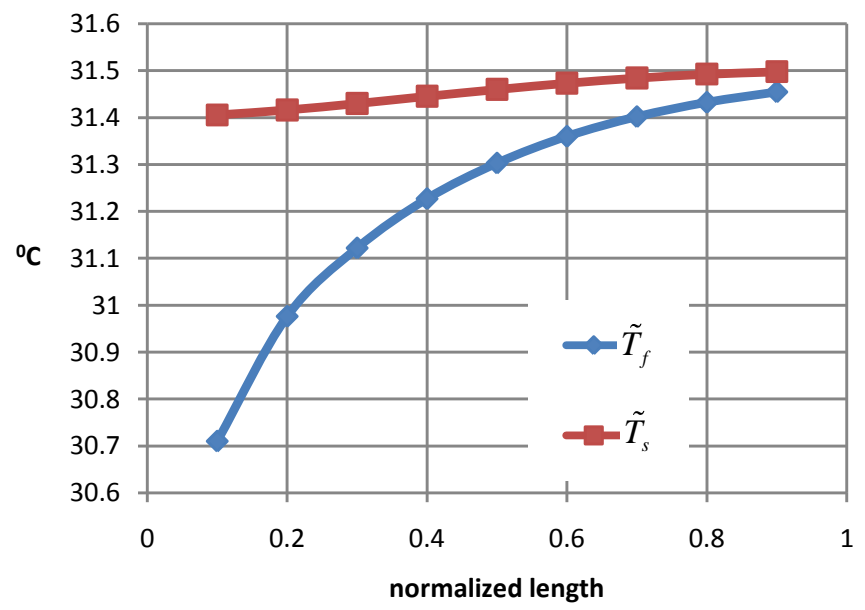

Figure 14: Development of averaged temperatures at $R_{h}=440.8$

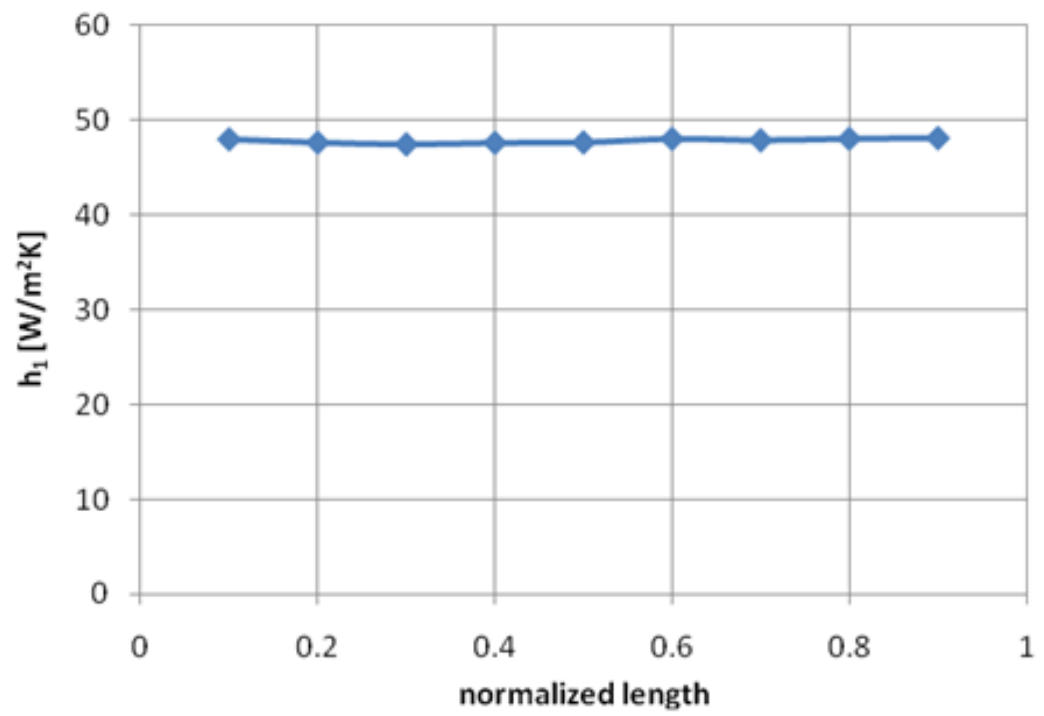

Figure 15: Local heat transfer coeficient at $\mathrm{Re}_{\mathrm{h}}=\mathbf{4 4 0 . 8}$ 
To explore how various heat sources and boundary conditions affect the heat transfer coefficient, different heating methods were chosen and a comparison of local heat transfer coefficients was made. The choices are constant temperature, constant heat flux and constant volumetric heat flux. The magnitude for each boundary condition is irrelevant since the heat transfer coefficient does not depend on how much heat is applied to the fluid, or in other words, by varying energy sources or sinks the temperature difference will adjust accordingly and therefore a boundary condition with a constant heat flux of $20 \mathrm{~W} / \mathrm{m}^{2}$ (BC: A), a constant temperature at $\mathrm{T}=70^{\circ} \mathrm{C}$ (BC: B) and a constant volumetric heat of $\dot{Q}=1.5 \mathrm{~kW} / \mathrm{m}^{3}$ (BC: C) were chosen. The values of the local heat transfer coefficients with different types of heating are shown on Figure 16 . The local Nusselt number, $\mathrm{Nu}=\mathrm{hd}_{\mathrm{h}} / \mathrm{k}$, was essentially a function only of the magnitude of the Reynolds and Prandtl numbers and is not dependent on the method of heating or boundary conditions.

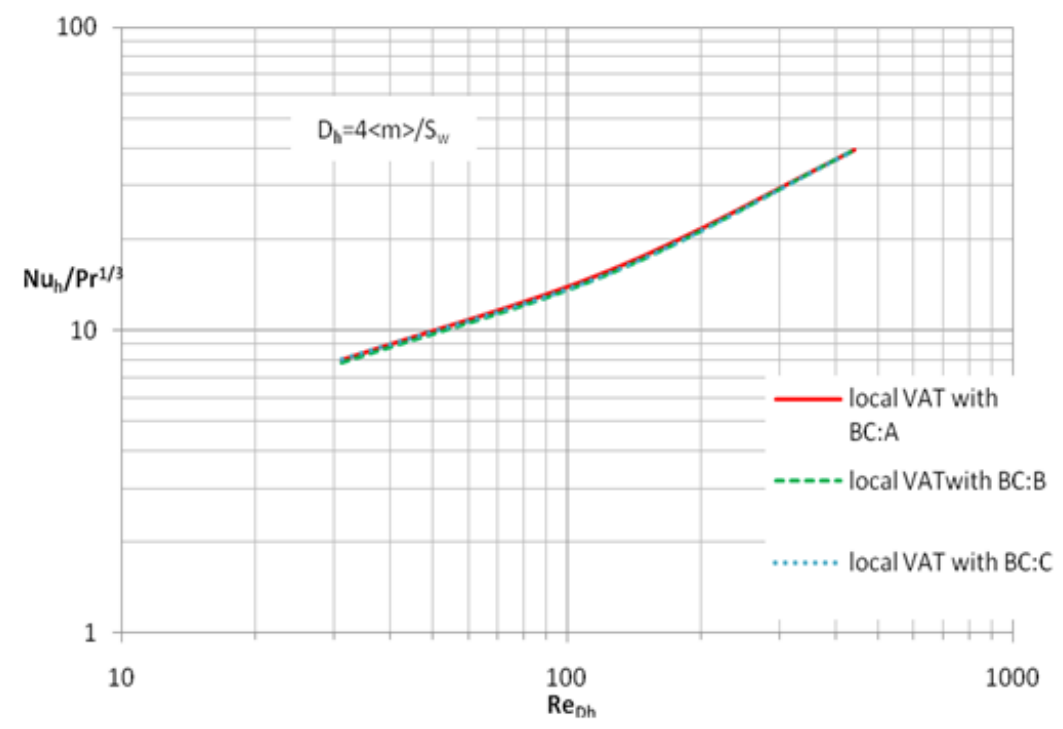

Figure 16: Local heat transfer coefficient with different types of heating. 


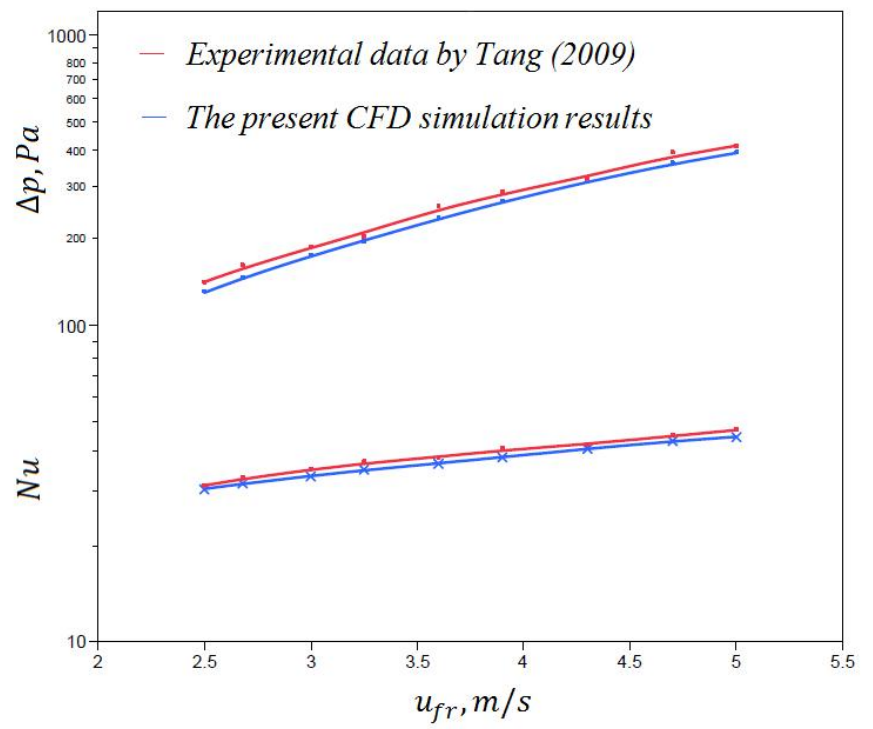

Figure 17: Comparison of CFD results with experimental data

A finned tube heat exchanger geometry was investigated by Feng, Hansen and Catton (2010) to make sure these simplifying aspects of our search for closure could be extended to other geometries. This was a two step process where the CFD results were first compared to experimental data, see Figure 17, and then the VAT closure terms were calculated.

Local values of the heat transfer coefficient were calculated using Eq. (52). The calculated values were again found to be independent of location and only a function of Reynolds number, see Figure 19. This is because the REV repeats itself in all directions leading to zero value for the fluctuation terms.

Local values are important because they are the only values that have a physical meaning when describing transport phenomena with VAT macro scale equations. The average values represent values that are valid over some length at a location where the flow is fully developed and they represent the total intra-phase heat transfer. The VAT local values, however, represent a local heat transfer coefficient that is based on a local average fluid temperature and a local 
average solid temperature. To be consistent with the VAT theory, only the local values of heat transfer closure can be used in calculations to accurately predict total heat transfer in a porous media, although average values are more important for comparison with experimental data.

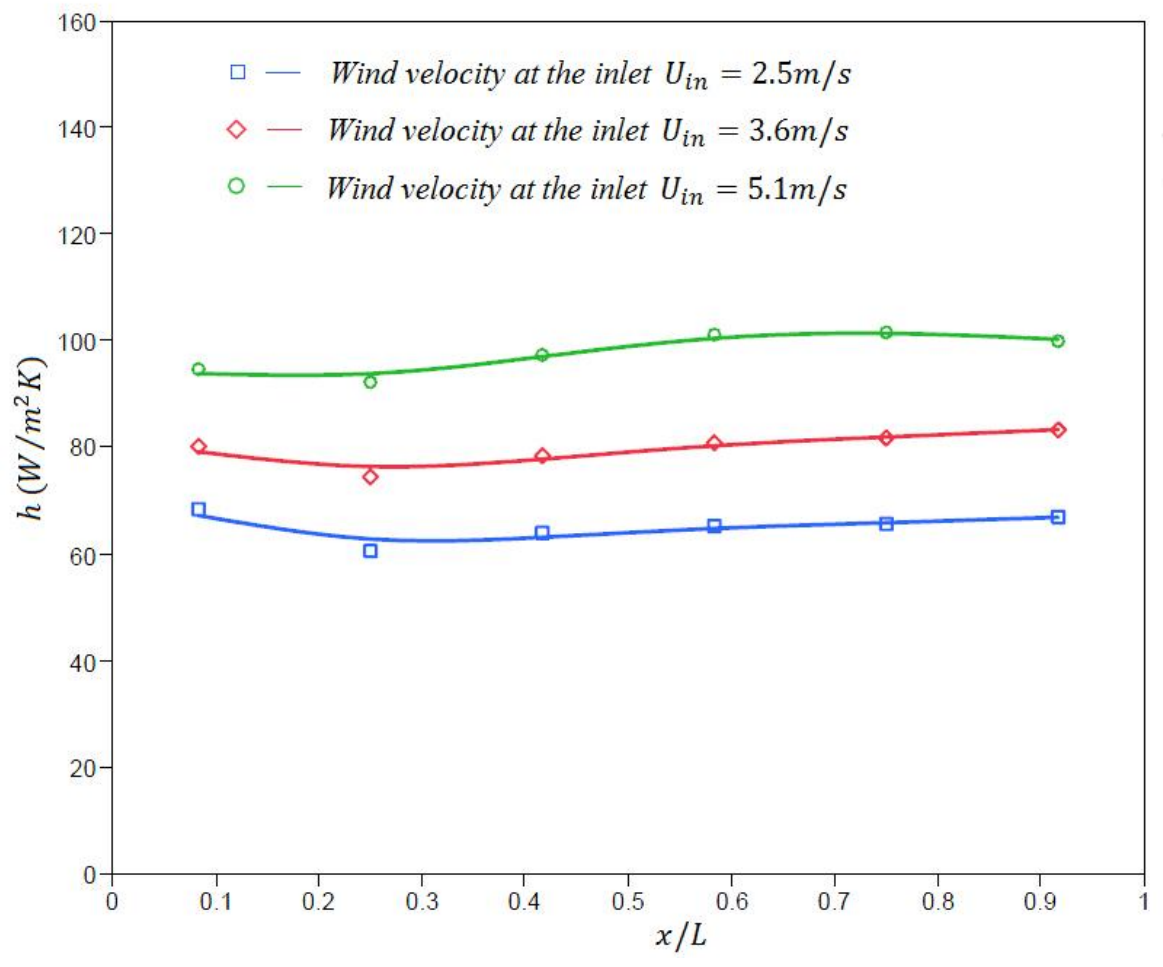

Figure 18: Local heat transfer coefficient at different flow rates from Zhou [2010]

An important aspect of what we have done is to show how CFD can be used to close the VAT based equations. There are a number of terms that contribute to the closure, see Eqs. (3942). They are calculated using results from the CFD solution to the problem. The local Nusselt number was found to depend only on the Reynolds number and Prandtl number and to be independent of the type of heating. The friction factor was shown to be almost independent of the geometry with a universal closure for pin fins in both inline and staggered configuration and for various packed bed porous media. A table showing closure values of A and B for various physical configurations with their range of validity is shown in Table 1. 
Most importantly, one can use heat transfer and friction factor data from any experiment that reports the values for fully developed flow providing the local average velocity and local values of the fluid and wall temperatures are used in their development. 


\section{Validation of CFD Heat Exchanger Model}

Before evaluating the friction factor and heat transfer coefficient from the output of a CFD code, the length of computational domain must be determined, so as to get a reasonable lower scale flow field solutions. A schematic diagram of a plain finned tube heat exchanger is shown in Figure 19. Usually, there are three or more rows of tubes which are arranged in-line or staggered.

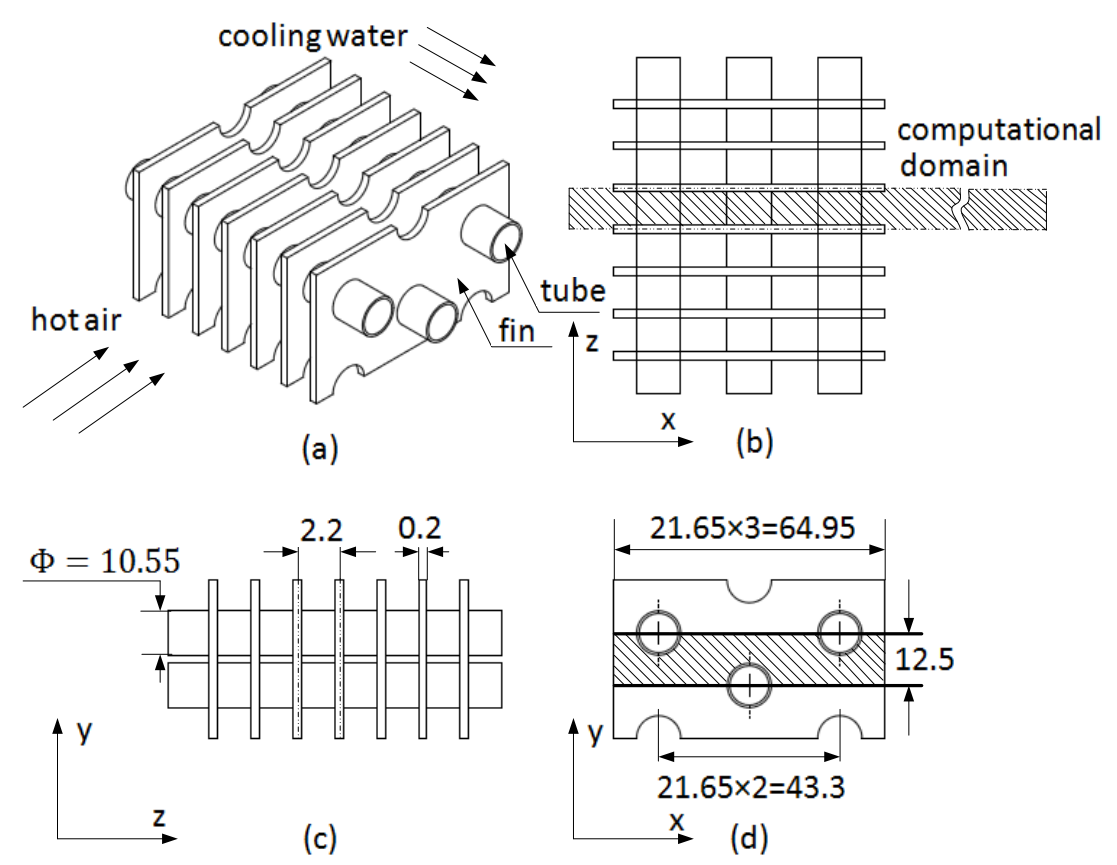

Figure 19: A schematic diagram of a plain plate finned tube heat exchanger.

The grid system for the configurations (take the 2-row one as an example) is shown in Figure 20. The actual computation domain was 7.5 times of the original heat transfer zone. The computational domain is not fully presented in order to save space. Because of the thickness of the fin, the air velocity profile at the entrance is not uniform. The computational domain is then extended upstream 1.5 times the stream-wise fin length so that a uniform velocity distribution 
can be ensured at the domain inlet. The computational domain is extended 5 times the streamwise fin length, so that at the outer flow boundary no flow re-circulation exits and the local oneway method can be used for the numerical treatment of the outer flow boundary condition. Also, it can be observed that in the extended parts, coarser grid was adopted to save the computing source and grid system with gradual variation in and after fin region was used, since the abrupt grid width change in the computing region should be avoided.

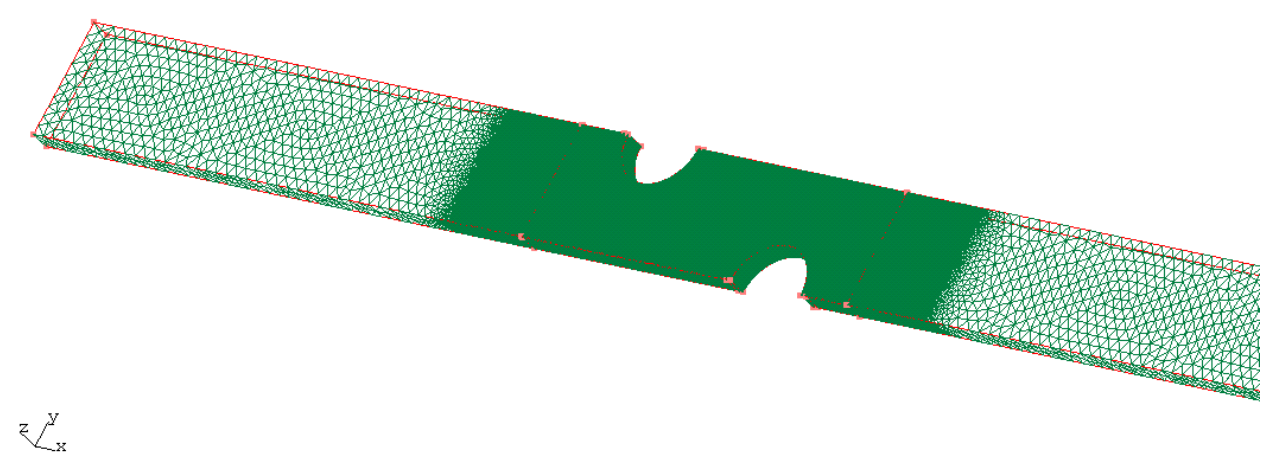

Figure 20: Grid system for CFD analysis

Grid independence tests were made carefully, and the numerical results obtained can be almost regarded as grid-independent. The numerical techniques and discretization schemes used in the code are a $2^{\text {nd }}$ order Monotone Upstream-centered Schemes for Conservation Laws (MUSCL) for convective terms in energy and momentum equation while for momentum and energy diffusion terms an accuracy weighted scheme is used. For the pressure correction a corrected version of semi-implicit method for pressure linked equations (SIMPLEC) is used.

\subsection{Parameter Definitions and Data Reduction}


Now definitions are presented for the characteristic quantities which will be used in the presentations of numerical results.

$$
\begin{aligned}
& R e_{D_{c}}=\frac{\rho u_{\max } D_{c}}{\mu} \\
& N u=\frac{h D_{c}}{\lambda} \\
& h=\frac{\Phi}{A_{o} \Delta T \eta_{o}} \\
& \Phi=\dot{m} c_{p}\left(T_{\text {in }}-T_{\text {out }}\right) \\
& \Delta T=\frac{T_{\max }-T_{\min }}{\log \left(T_{\max } / T_{\min }\right)} \\
& \eta_{o}=1-\frac{A_{f}}{A_{o}}\left(1-\eta_{f}\right) \\
& A_{f}=N\left[P_{t} P_{l}-\pi\left(\frac{D_{c}}{2}\right)^{2}\right] \\
& A_{o}=N\left[P_{t} P_{l}-\pi\left(\frac{D_{c}}{2}\right)^{2}+\frac{1}{2} \pi D_{c}\left(F_{p}-\delta_{f}\right)\right] \\
& f=\frac{\Delta p}{\frac{1}{2} \rho u_{\max }^{2}} \cdot \frac{D_{c}}{L} \\
& \Delta p=p_{\text {in }}-p_{\text {out }}
\end{aligned}
$$

where $u_{\max }$ is the mean velocity of the minimum transverse area, $D_{c}$ is the fin collar outside diameter, $\dot{m}$ is the mass flux of the air, $T_{\text {out }}, T_{\text {in }}$ are the fluid bulk temperature of outlet and inlet of the computation domain, respectively, $T_{\max }=\max \left(T_{\text {in }}-T_{w}, T_{\text {out }}-T_{w}\right), \quad T_{\min }=$ $\min \left(T_{\text {in }}-T_{w}, T_{\text {out }}-T_{w}\right), \eta_{f}$ is the fin efficiency and $\eta_{o}$ stands for the overall fin efficiency. $\Phi$ is the actual heat transfer rate between air and the fin surface. 
As has been discussed above, the computation is of a conjugated type in which the fin efficiency has to be determined during the computations and cannot be obtained in advance. According to heat transfer theory (Kupprn, 2000; Bejan, 2003), the fin efficiency is defined as the actual heat transfer rate for the fin and tube divided by the heat transfer rate for the fin and tube when the fin is at the same tube temperature $T_{w}$. In our numerical simulation the fin efficiency $\eta_{f}$ is computed by the approximation method described by Schmidt (1949) as follows.

$$
\eta_{f}=\frac{\tanh (m r \phi)}{m r \phi}
$$

Where

$$
\begin{gathered}
m=\sqrt{\frac{2 h_{o}}{\lambda_{f} \delta_{f}}} \\
\phi=\left(\frac{R_{e q}}{r}-1\right)\left[1+0.35 \ln \left(\frac{R_{e q}}{r}\right)\right]
\end{gathered}
$$

For staggered tube layout:

$$
\frac{R_{e q}}{r}=1.27 \frac{X_{M}}{r}\left(\frac{X_{L}}{X_{M}}-0.3\right)^{1 / 2}
$$

For inline tube layout, or 1-row fin-and-tube heat exchanger,

$$
\begin{gathered}
\frac{R_{e q}}{r}=1.28 \frac{X_{M}}{r}\left(\frac{X_{L}}{X_{M}}-0.2\right)^{1 / 2} \\
X_{L}=\frac{\sqrt{\left(P_{t} / 2\right)^{2}+P_{l}^{2}}}{2} \\
X_{M}=P_{t} / 2
\end{gathered}
$$

For the presentation of numerical results in term of field synergy principle, the following quantity is introduced:

$$
M=\frac{\sum(|\vec{U}||\operatorname{grad} T|)}{n}
$$


where $n$ is the number of control volume covering the fin region. Local intersection angle between the velocity and temperature gradient:

$$
\theta=\cos ^{-1} \frac{u \frac{\partial T}{\partial x}+v \frac{\delta T}{\delta y}+w \frac{\partial T}{\partial z}}{|\vec{U}||\operatorname{grad} T|}
$$

Average intersection angle of the computation domain which is defined by

$$
\theta_{m}=\frac{\sum \theta_{i, j, k} d v_{i, j, k}}{\sum d v_{i, j, k}}
$$

where $d v_{i, j, k}$ is the volume element of the control volume $(i, j, k)$.

To verify the computational model and the method adopted in numerical simulation for the finned tube heat exchanger, preliminary computations were first conducted for a three-row plain finned tube heat exchanger. The dimensions we used are the same as those of the heat exchanger measured by Kang (1994).

The Nusselt number and friction factor reduced from simulation results were compared with the correlations by Kang (1994) and the correlations by Wang (2000) as shown in Figure 8. It should be noted that the correlations developed by Wang is based a total of 74 samples and the proposed heat transfer correlation can describe $88.6 \%$ of the database within $\pm 15 \%$, while the proposed friction correlation can correlate $85.1 \%$ of the database within $\pm 15 \%$. From Figure 8 we can see that the maximum deviation in friction factor and Nusselt number are $10.4 \%$ and $11.9 \%$ with the average deviation being around $6 \%$ and $5.5 \%$ respectively. Our predicted results and the experimental data agree very well, which shows the reliability of the physical model and the adopted numerical method. 


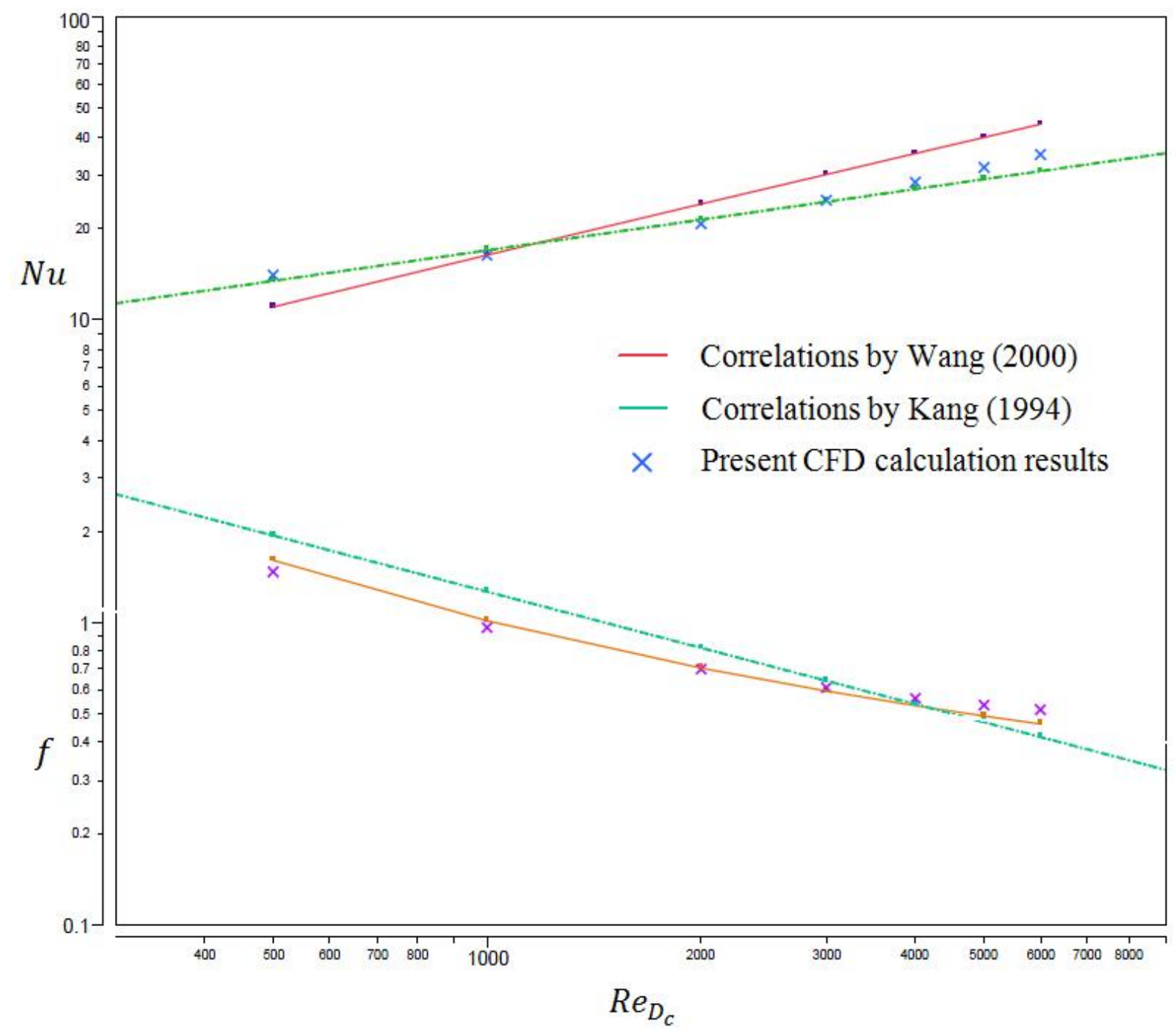

Figure 21: Comparison between CFD results, correlations and experimental data.

Our CFD simulation results are shown in Figure 10, which presents the variation of average $N u$ and $f$ with the increase of tube row number $N$. It can be seen from Figure 10 (b) that the variation of friction factor with $N$ is quite small, and when the row number is larger than $3, f$ could be considered independent of $N$. This result agrees well with that of the experiments by Wang (1996) and Wang (2000). Figure 10 (a) shows that Nusselt number decreases with the increasing of tube row number $N$, and when $N>4$, the decreasing trend slows down. 


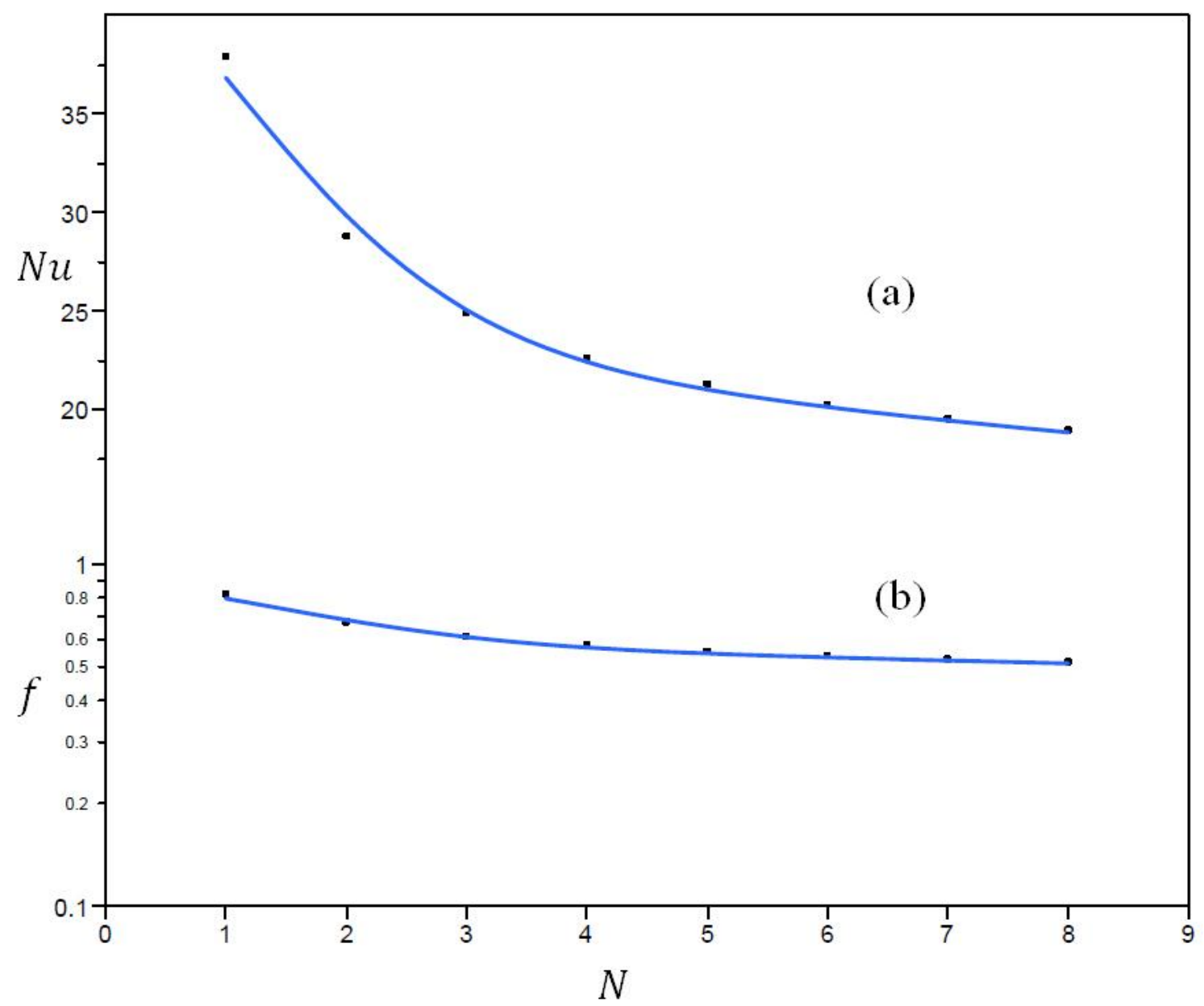

Figure 22: Variation of $\mathrm{Nu}$ and $f$ with tube row number by CFD simulation.

Here we will analyze this phenomenon from the point view of Field Synergy Principle. Figure 11 (a) and (b) show the variation of $\frac{M}{M_{0}}$ and $\theta_{m}$ with the increase with tube row number, where $M_{0}$ is the $M$ value for one-row case. As can be seen, the average product of $|\overrightarrow{\mathrm{U}}|$ and $|\operatorname{gradT}|$ decreases with the increasing of $N$, but the decreasing trend doesn't decreases as the Nusselt number does. However, we can find that the intersection angle between air flow velocity and temperature gradient increases with the increasing tube row number and the increasing trend slows down when $N>4$, which agrees with the variation trend of Nusselt with $N$ perfectly. So it could conclude that the heat transfer and fluid flow approach fully developed conditions when 
the number of tube rows is greater than six. Wang (1996) and Xie (2009) also got the same conclusion.

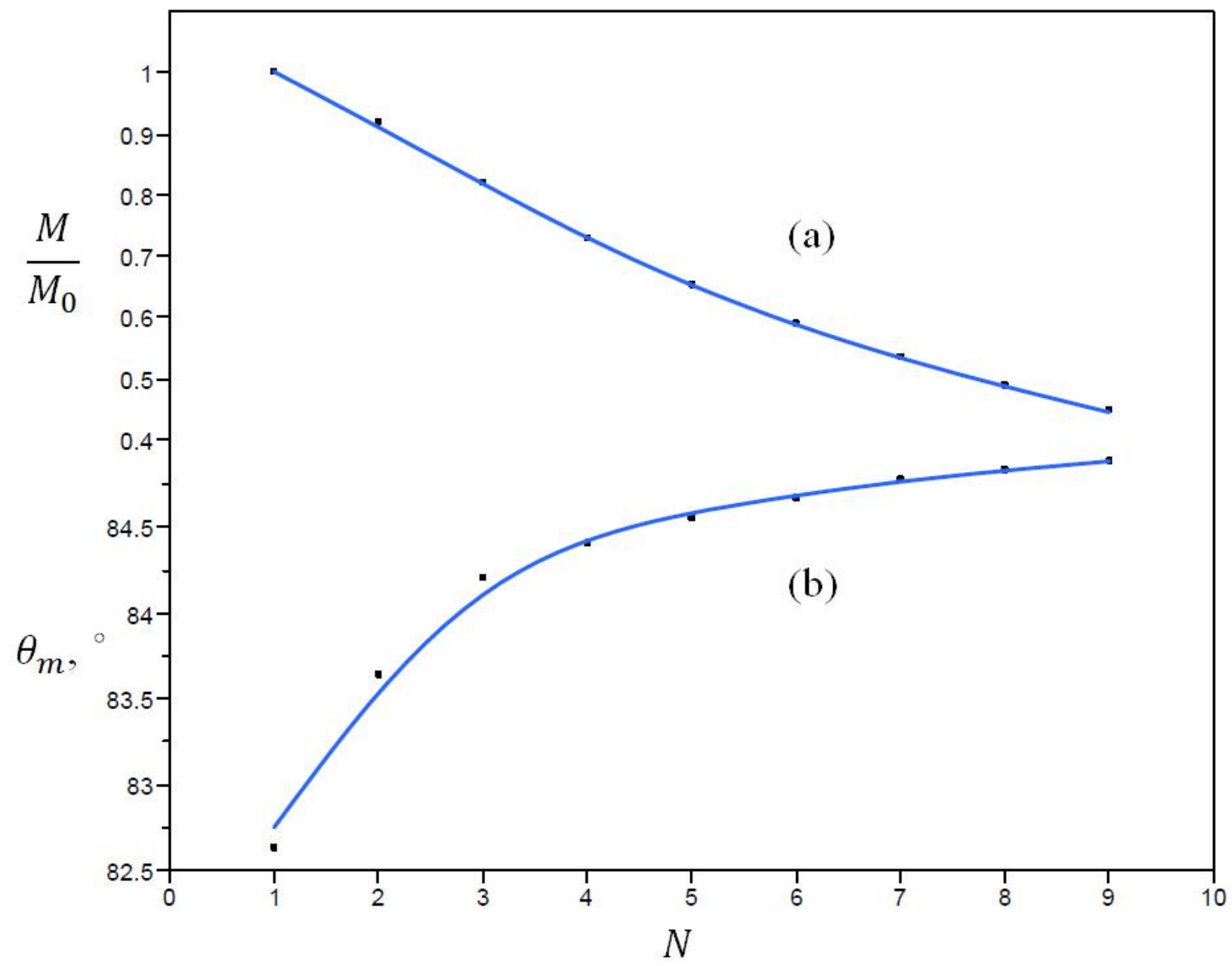

Figure 23: Variation of $M$ and $\theta_{\mathrm{m}}$ with tube row number $N$.

To get a more vivid impression, the local distributions of isothermals, velocity vector, and intersection angle in the middle section in the $\mathrm{x}-\mathrm{y}$ plane for $R e_{D_{c}}=3000$ are presented below for two-row case and nine-row case, respectively. From Figure 12 and Figure 13, we can find that at the inlet of the heat exchanger, the flow velocity is almost perpendicular to the isothermal curves. As the tube row number increase, the flow velocity is becoming parallel to the isothermal 
which means that the synergy was deteriorated. So, it is clear that as the number of tube rows increases, the percentage of the area which has deteriorated synergy increases.
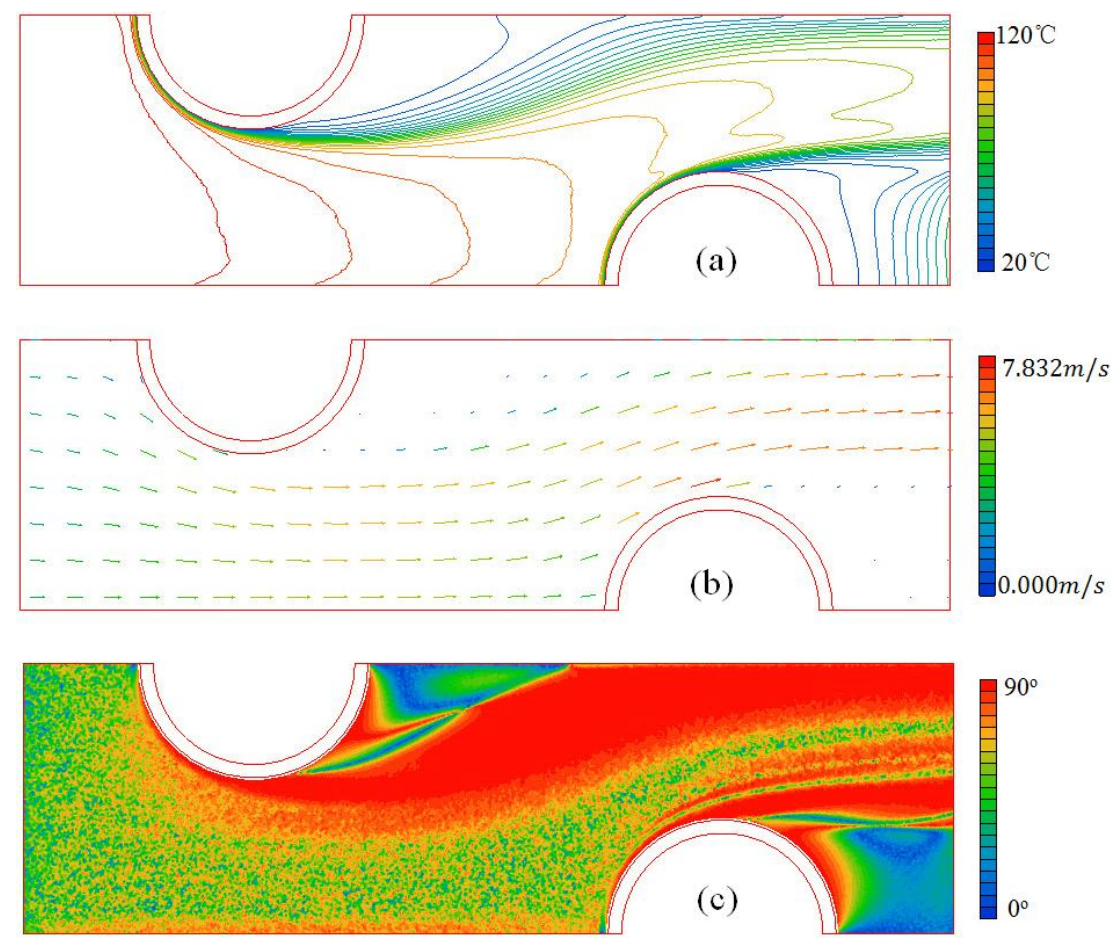

Figure 24: $\mathrm{N}=2$ Temperature, Velocity and Intersection angle profiles

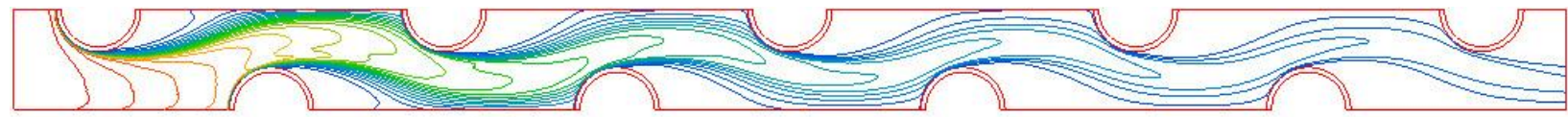

(a)

$120^{\circ} \mathrm{C}$

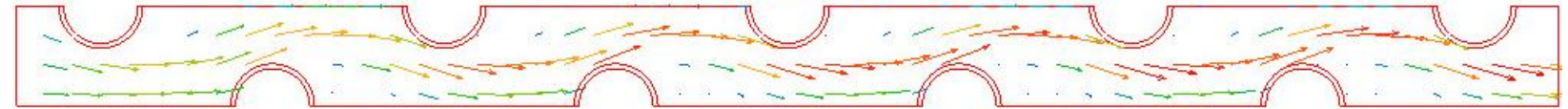

(b) $0.000 \mathrm{~m} / \mathrm{s} \square \Pi\|\|\|\|\|\| \| \amalg=8.2491 \mathrm{~m} / \mathrm{s}$

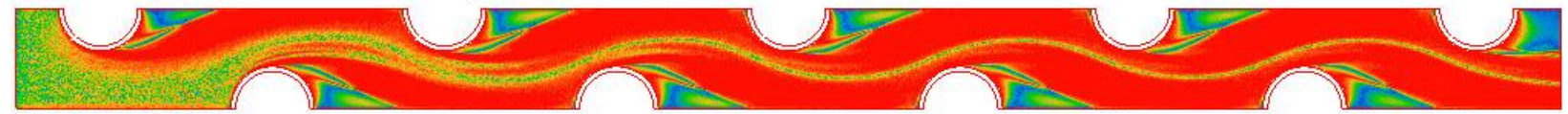

(c)

$0^{\circ}$ पW

Figure 25: $\mathrm{N}=9$ Temperature, Velocity and Intersection angle profiles

It should be noted that, all the above discussion is based on average values over the whole simulation domain, while to use volume average theory to optimize the heat exchanger, we are more concerned about the local values of the selected representative elementary volume (REV). 
So, although we have got the conclusion that when the number of tube rows is larger than six, the flow field and heat transfer could be considered as fully developed, we cannot say that six longitudinal tube pitches is the length of computational domain we need to get a reasonable lower scale flow field and heat transfer solutions. In-depth observations should be carried out on the variation of concerned parameters over every single REV along with the air flow direction. Thus, we divided the computational domain of 7-row case, 8-row case, and 9-row case into 7, 8, and 9 REV respectively, calculated the Nusselt numbers, $M$ values and intersection angles of the total 24 REVs, and plotted the results in Figure 14. Seven conclusions could be made from the graph: Where are the conclusions

The averaged local $\mathrm{M}$ value of a single REV decreases along with the direction of air flow. This is why the magnitude of $\mathrm{M}$ averaged over the whole domain decreases as the number of tube rows increase. Since the values of $\mathrm{M}$ of all three cases collapse to a single curve, the number of tube rows has no effect on M. The effect of tube row number on Nusselt number is through the variation of synergy intersection angle. The averaged local Nusselt number decreases along with the direction of air flow and the decreasing trend decreases when $\mathrm{N}>4$. The averaged local intersection angle increases along with the direction of air flow and the increasing trend decreases when $\mathrm{N}>4$. 


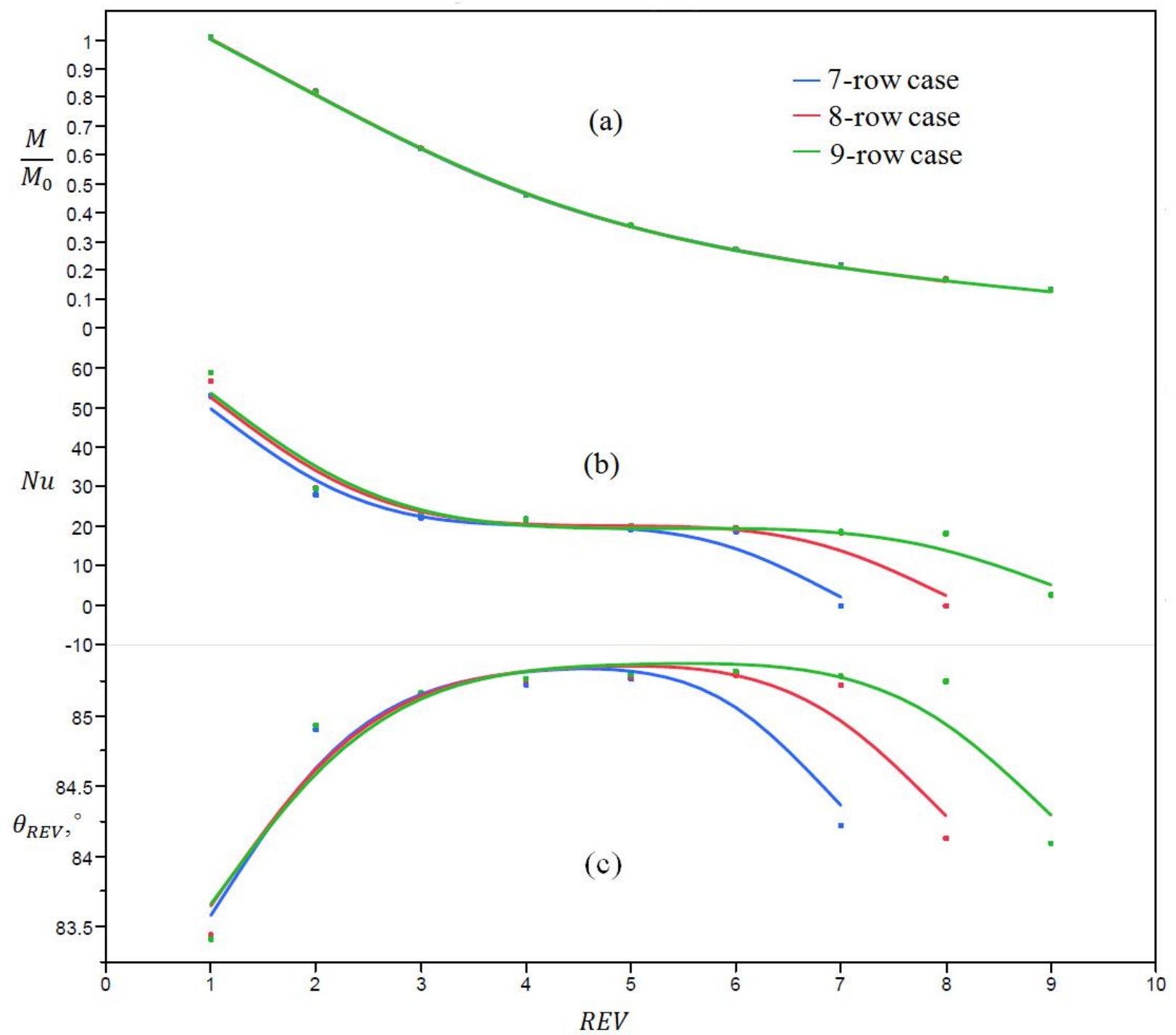

Figure 26: Variation of $M, N u$ and $\theta_{\mathrm{m}}$ of each REV.

The averaged local $\mathrm{M}$ value of a single REV decreases along with the direction of air flow. This is why the magnitude of $\mathrm{M}$ averaged over the whole domain decreases as the number of tube rows increase. Since the values of $\mathrm{M}$ of all three cases collapse to a single curve, the number of tube rows has no effect on M. The effect of tube row number on Nusselt number is through the variation of synergy intersection angle. The averaged local Nusselt number decreases along with the direction of air flow and the decreasing trend decreases when $\mathrm{N}>4$. The averaged local 
intersection angle increases along with the direction of air flow and the increasing trend decreases when $\mathrm{N}>4$.

$\mathrm{N}>4$ is the bottom line to get a believable local value averaged over a single REV, which means that the length of computational domain should not be less than five REV (we recommend six) from this point of view. The Nu number and intersection angle of the last REV is unusual, this may due to that the air flow over a backward step when it flow from the last REV to the extended domain. So the value of the last REV shouldn't be used to calculate the colure for VAT based model. Considering the fully-development issue and the outlet effect, at least 5+1 REVs should be calculated and the local value of the fifth REV would be used to obtain the closure. We recommend that $5+2+2 \mathrm{REV}$ s being simulated and the closure being obtained by averaging the local value over the sixth and seventh REVs.

After determining the length of the computational domain we need to simulate, so as to get a reasonable results, the VAT based formulas for the friction factor and heat transfer coefficient will be integrate over the interface between fluid and solid. The VAT governing equations for fin-tube heat exchanger will be closed by using CFD results.

\subsection{Experimental Determination of Heat Transfer}

Determining the heat transfer coefficient in a porous medium experimentally is challenging due to its geometry. Other transient experimental techniques have been developed for this purpose. One such technique employs a step change in gas phase temperature, but this technique can prove to be difficult to implement in practice. Oscillatory flows have also been investigated but suffer from both data reduction difficulties and the lack of flow rate resolution. The technique described in this paper determines the average heat transfer coefficient from 
measurement of the fluid temperature response to a step change in the volumetric heating rate of the solid phase. It is desired to validate this method by comparing its results for a packed bed of spheres to established correlations.

Steady state and one-dimensional flow conditions are achieved in a randomly packed bed of spheres. The solid phase is subjected to a step change in volumetric heat generation rate from an induction heater. The transient gas phase exit temperature response is measured until steady state thermal conditions are achieved. The solid phase is AISI 1010 steel and the fluid is air. The tube in which the packed bed is contained is a clear pvc, providing a nearly adiabatic wall condition. Polypropylene spheres upstream of the test section serve to hydrodynamically fully develop the flow before it enters the test section. The test section is composed of 0.19 inch diameter steel spheres. The cross-sectional diameter of the test section is 2.65 inches and its length is 1.90 inches. Two thermocouple rakes are positioned upstream and downstream of the porous medium to measure the transient gas temperature response. A rotameter measures the gas flow rate. A differential pressure transducer records the pressure loss. Care is taken to minimize lateral boundary effects both thermally and hydrodynamically. Figure 27 and Figure 28 show the experimental test section.

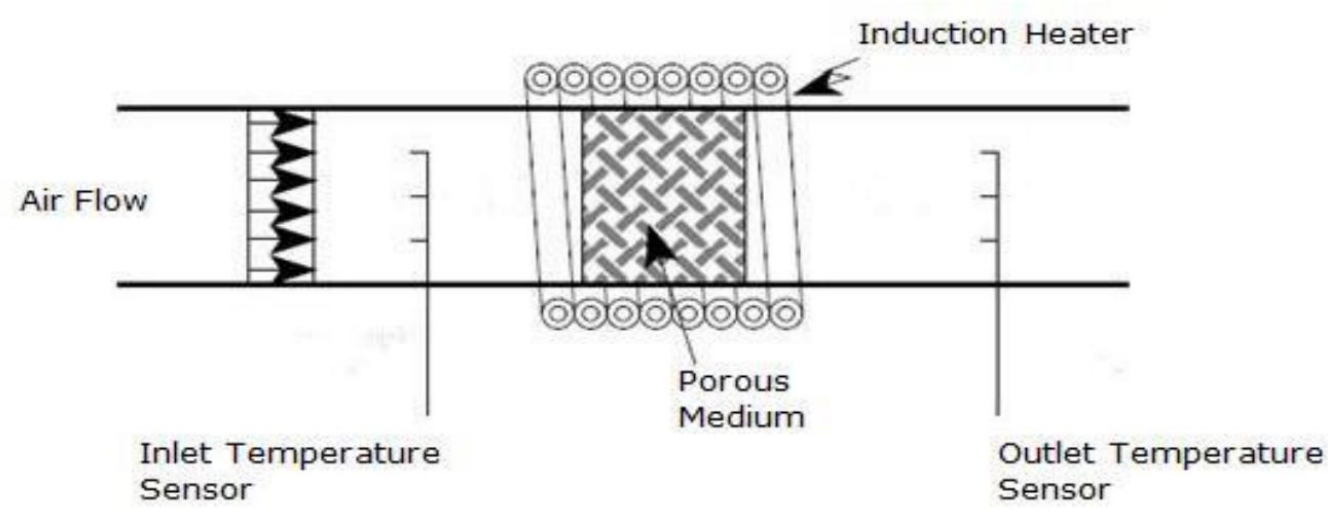

Figure 27: Experimental section for bed of spheres 


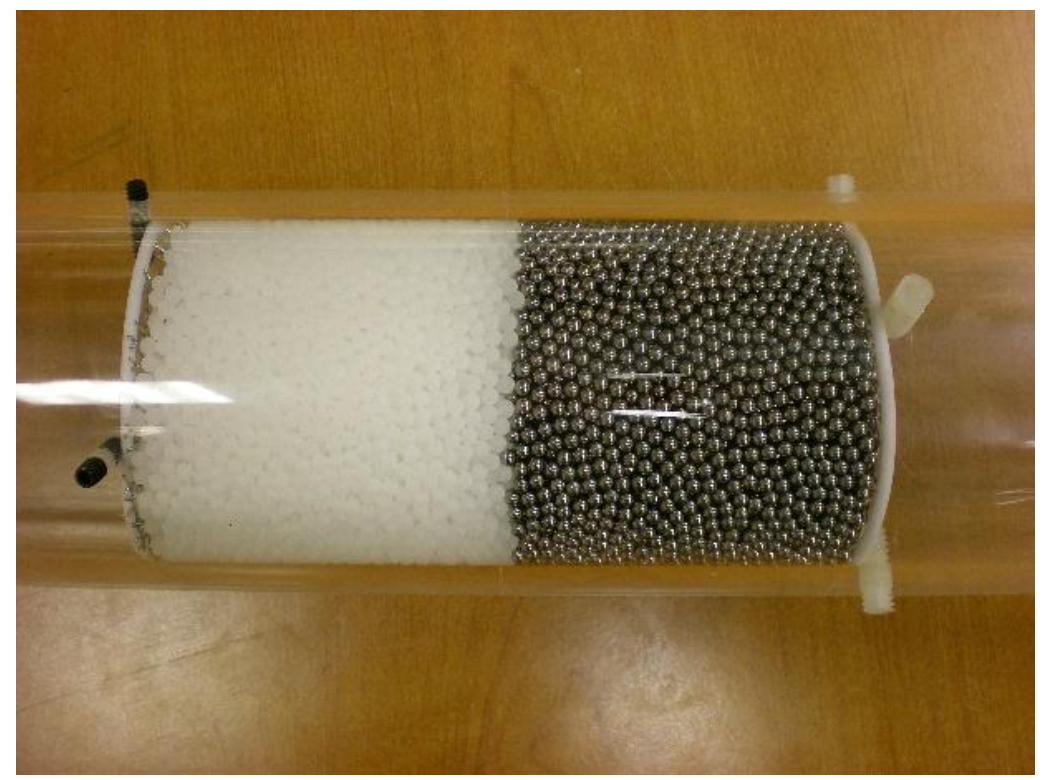

Figure 28: Packed bed of spheres test section

The heat transfer coefficient for a given heat exchanger design can be determined non-intrusively intrusively through a method developed by Jones and Catton (2001). In this method the fluid flows through the heat exchanger while induction heating provides a step change in the solid phase's heat generation rate. The fluid's temperature response is recorded. Based upon the volume-averaged equations, the heat transfer coefficient can be determined through a computational procedure, since the outlet temperature response is dependent upon Nu number as shown in Figure 29: Dimensionless gas exit temperature versus time, with varying Nu.

The gas phase temperature response data for a given flow rate is fed into our code. The code iterates the simulation until it matches the experimental data, at which point the Nusselt number for the specified flow rate is known. An example of the simulation matching the code can be seen in Figure 31.

- An equation set based on volume averaging theory can be numerically solved to determine the heat transfer coefficient from the fluid temperature response. 
The heat transfer coefficient is determined by comparing the results of a numerical simulation based on volume averaging theory with the experimental results. Given the inputs, which are simply the basic material properties, flow condition, and the experimental results, the error tolerance in the experimental and numerical results is determined. When this error is too large, the Nusselt number in the numerical simulation is adjusted, and the error tolerance is looked at again. As long as the error is too large the Nusselt number will continually be adjusted. Eventually, the error tolerance will be small enough so that we can conclude that the most recent Nusselt number is an accurate approximation of the real Nusselt number. Of course, with the Nusselt number known, the heat transfer coefficient may then be determined. Air flow through the test section at various pore Reynolds numbers between 150 and 500 while the test section was volumetrically heated the solid phase at some unknown rate. The gas temperature response for each flow rate was recorded, see Figure 30.

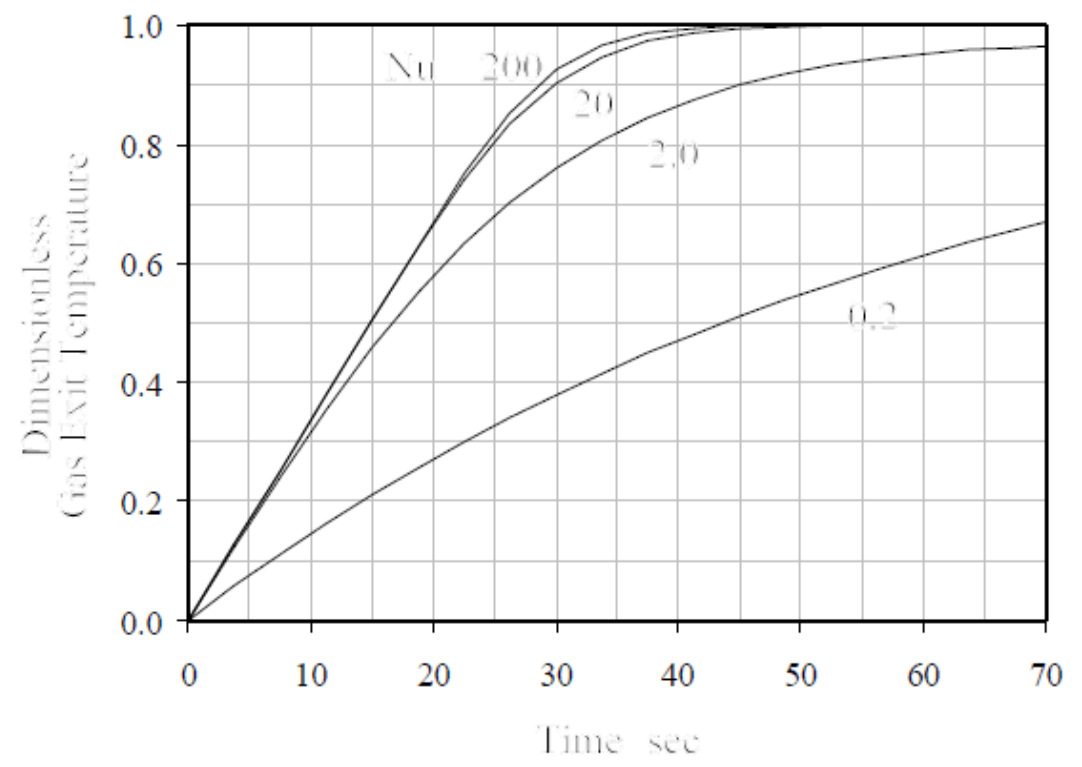

Figure 29: Dimensionless gas exit temperature versus time, with varying Nu. 
The gas phase temperature response data for a given flow rate is fed into our code. The code iterates the simulation until it matches the experimental data, at which point the Nusselt number for the specified flow rate is known. An example of the simulation matching the code can be seen in Figure 31.

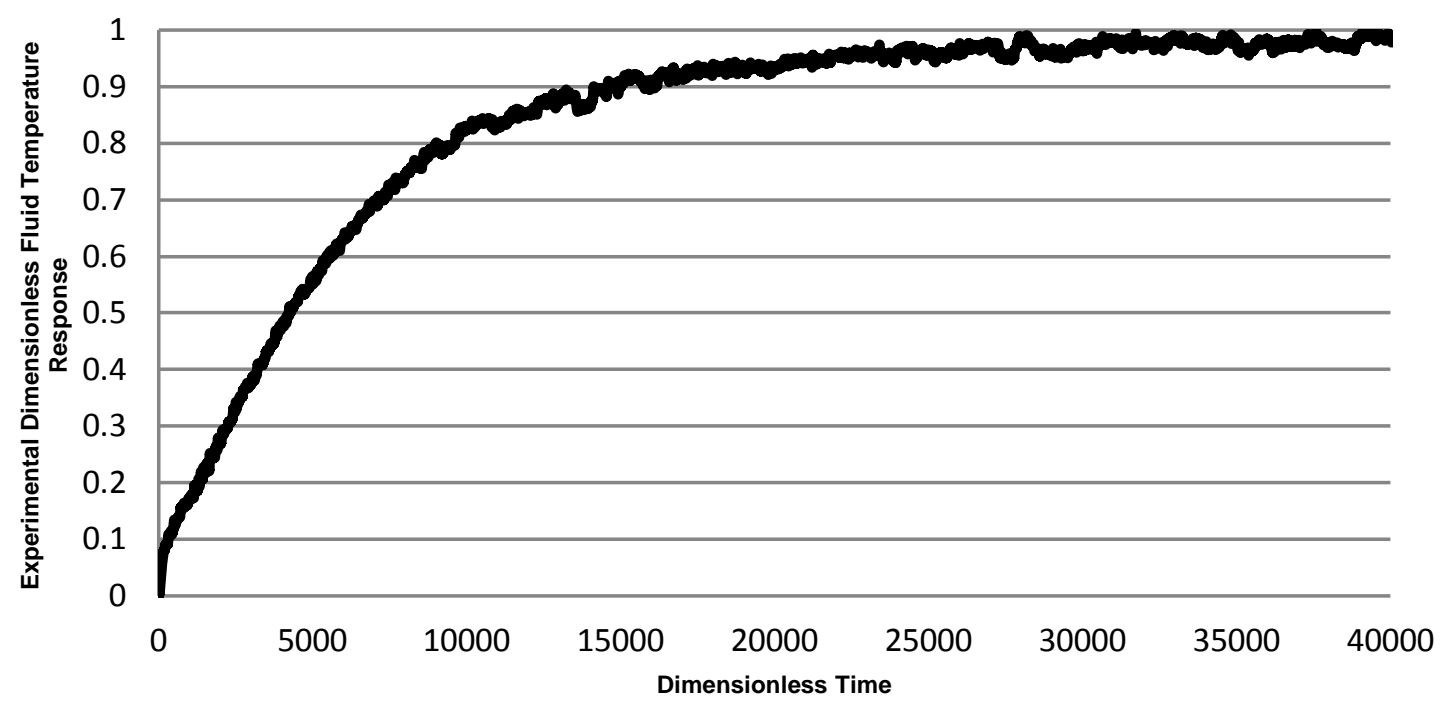

Figure 30: Example of a Transient Gas Phase Temperature Response 


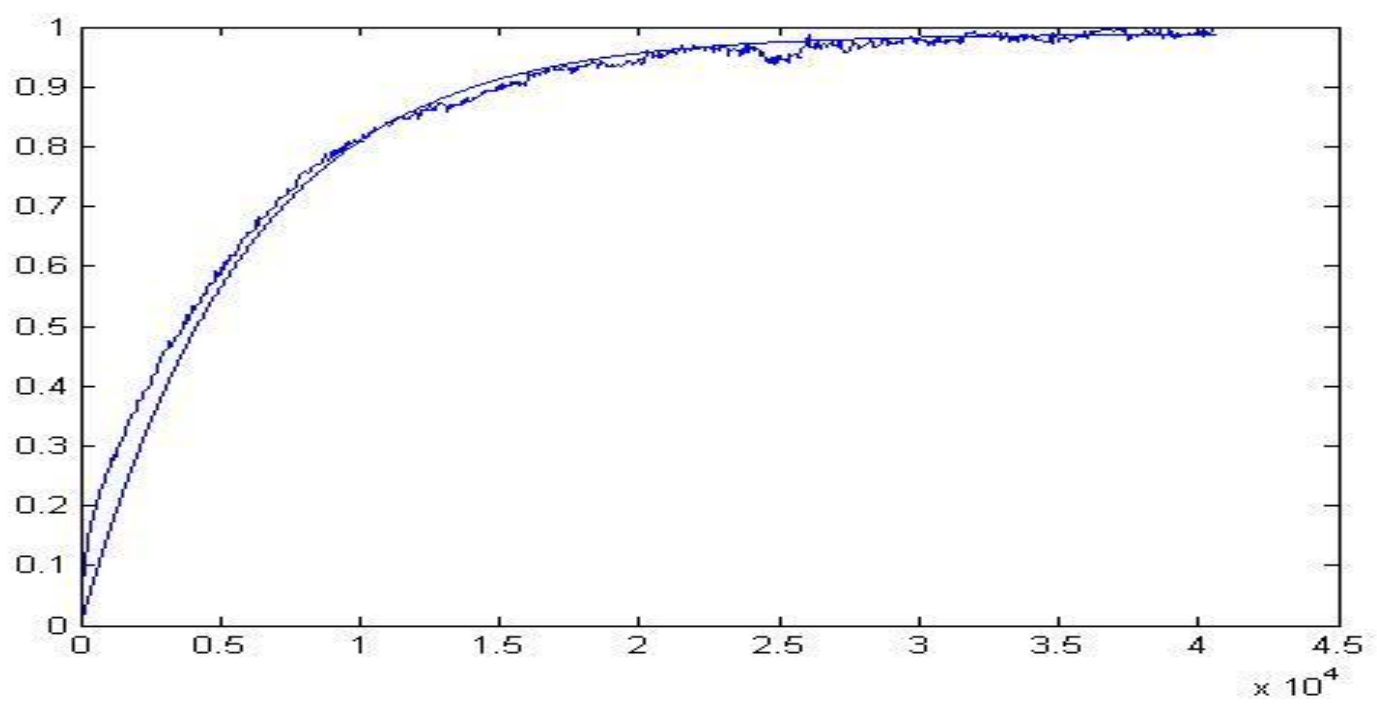

Figure 31: Gas Phase Temperature Response: Experimental Versus Simulation.

In Figure 31 the experimental data is the "rough" line while the simulation is the smooth line. Note that both coordinate axes have been non-dimensionalized. The vertical axis, the gas phase temperature response, has been scaled between zero and one. The horizontal axis, the time, has been non-dimensionalized and is in units of a so called "characteristic" time. The characteristic time is defined as $\tau=\frac{\mathrm{L}}{\mathrm{u}_{0}}=\frac{\varepsilon \mathrm{L}}{\mathrm{U}}$. Using this technique, the heat transfer coefficient of any irregular porous surface can be measured.

\subsection{Heat Exchanger Optimization Modeling}

Usually, there are three or more rows of tubes which are arranged in-line or staggered, and in most practical manufacturing, heat exchanger cores with a large number of tube-rows are often used. It is very difficult to perform a direct simulation of the whole heat exchanger with a 3-D model for the Reynolds numbers of interest even though high speed computers with large memory have emerged, because the large number of grids needed will be very time-consuming and our goal is multi-parameter optimization. A fast running model has been built basing on the 
volume averaging theory (VAT). Complex closure terms arise that must be evaluated and this is done using experimental data or CFD results.

Here we consider the hot air flow as well as the cooling water flow through the tube as porous flow, in which the term porous is used in a broad sense. Applying VAT to the transport equations, the flow variables are averaged over a representative elementary volume (REV) of the heat exchanger. The averaged equations have a form of porous media flow equations, where each phase and its properties are separately defined over the whole simulation domain.

The VAT equations for flow and heat transfer were first developed by Whitaker (1986). Later, a plan for heat transfer optimization was described by Travkin and Catton (1994). Hu (2002) and Vadnjal (2009) further developed the application of the theory to optimize heat transfer in porous media. In this section, the momentum and energy equations for the air side, water side and solid part are presented below. The resulting momentum equations for the two fluids are:

Air side:

$$
-\frac{1}{\rho_{1}} \frac{\partial\left\langle\bar{p}_{1}\right\rangle_{f}}{\partial x}+\frac{\partial}{\partial z}\left(\left\langle m_{1}\right\rangle\left(\tilde{v}_{T_{1}}+v_{1}\right) \frac{\partial \widetilde{u}_{1}}{\partial z}\right)+c_{d_{1}} S_{w_{1}} \frac{\widetilde{u}_{1}^{2}}{2}=0
$$

Water side:

$$
-\frac{1}{\rho_{2}} \frac{\partial\left\langle\bar{p}_{2}\right\rangle_{f}}{\partial z}+\frac{\partial}{\partial x}\left(\left\langle m_{2}\right\rangle\left(\tilde{v}_{T_{2}}+v_{2}\right) \frac{\partial \widetilde{w}_{2}}{\partial x}\right)+c_{d_{2}} S_{w_{2}} \frac{\widetilde{w}_{2}^{2}}{2}=0
$$

Here, the subscripts 1 and 2 indicate values on the air side and water side respectively. $\langle f\rangle_{f}$ means the superficial average of the function $f$. The superscript - and $\sim$ indicate an average of turbulent values and a value averaged over the representative volume respectively. $\rho$ is the density. $p$ is the pressure of the fluids with turbulent terms averaged over the reference volume. $\langle m\rangle$ is the porosity in the averaging volume. $\tilde{v}_{T}$ and $v$ are the averaged turbulent viscosity and the kinematic viscosity. $c_{d}$ is the drag coefficient. $S_{w}$ is the specific surface. $u$ and $w$ are the 
velocities in the $\mathrm{x}$-direction and $\mathrm{z}$-direction respectively, with turbulent terms averaged over the reference volume.

Because we are dealing with a conjugate problem, the thermal energy equations for both the solid and fluid states are required for the simulation. For the fluid phases, the VAT formulations used in the simulation is

Air side:

$$
\left\langle m_{1}\right\rangle \rho_{1} \tilde{\bar{u}}_{1} c_{p_{1}} \frac{\partial \tilde{\bar{T}}_{1}}{\partial x}=h_{1} S_{w_{1}}\left(\bar{T}_{s}-\tilde{\bar{T}}_{1}\right)
$$

Water side:

$$
\left\langle m_{2}\right\rangle \rho_{2} \widetilde{\bar{w}}_{2} c_{p_{2}} \frac{\partial \tilde{\bar{T}}_{2}}{\partial z}=h_{2} S_{w_{2}}\left(\tilde{T}_{s}-\tilde{\bar{T}}_{2}\right)
$$

Here $c_{p_{1}}$ and $c_{p_{2}}$ are the specific heats of the fluids respectively. $T_{1}$ and $T_{2}$ are the fluid temperatures with turbulent terms averaged over the reference volume. $T_{s}$ is the temperature of the solid. $h$ is the averaged heat transfer coefficient. For the solid phase, the VAT based energy equation is:

$$
\begin{aligned}
\frac{\partial}{\partial x}\left[\left(1-\left\langle m_{1}\right\rangle\right.\right. & \left.\left.-\left\langle m_{2}\right\rangle\right) k_{s} \frac{\partial \tilde{T}_{s}}{\partial x}\right]+\frac{\partial}{\partial z}\left[\left(1-\left\langle m_{1}\right\rangle-\left\langle m_{2}\right\rangle\right) k_{s} \frac{\partial \tilde{T}_{s}}{\partial z}\right] \\
& =h_{1} S_{w_{1}}\left(\bar{T}_{s}-\tilde{\bar{T}}_{1}\right)+h_{2} S_{w_{2}}\left(\tilde{T}_{s}-\tilde{\bar{T}}_{2}\right)
\end{aligned}
$$

Here, $k_{s}$ is the thermal conductivity of the solid. $\left(1-\left\langle m_{1}\right\rangle-\left\langle m_{2}\right\rangle\right)$ can be considered as the averaged "blockage".

\subsection{Computer Code}


With the closure correlations, the VAT equations can be solved discreetly. Using FORTRAN, a computer code was written to solve the VAT equations at every point in a 2 dimensional representation of a heat exchanger. The code solves each REV as spatially and time averaged. The values of porosity and surface wetted are found from the geometry for each grid point, and from those, the hydraulic diameter is defined. With the hydraulic diameter locally defined, the value of friction factor and Nussult number can be found locally. The values of velocity and temperatures of both fluids can then be found at every grid point. The temperature and velocity are found from conservation equations for momentum and energy.

The governing equations for Fluid one and Fluid two are shown in equations 1-4 and the solid is shown in equation 5. The volume averaged energy equations for the solid phase is shown below.

$$
\begin{aligned}
& \frac{\partial}{\partial x}\left(\left(1-\left\langle m_{S}(x, z)\right\rangle\right) k_{t S}(x, z) \frac{\partial T_{S}(x, z)}{\partial x}\right)+\frac{\partial}{\partial z}\left(\left(1-\left\langle m_{S}(x, z)\right\rangle\right) k_{t S}(x, z) \frac{\partial T_{S}(x, z)}{\partial z}\right) \\
& =\tilde{\alpha}_{t H}(x, z) S_{w H}(x, z)\left(T_{S}(x, z)-\tilde{\bar{T}}_{H}(x, z)\right)+\tilde{\alpha}_{t C}(x, z) S_{w C}(x, z)\left(T_{S}(x, z)-\tilde{\bar{T}}_{C}(x, z)\right)+\left(1-\left\langle m_{S}(x, z)\right\rangle\right) \tilde{S}_{T}
\end{aligned}
$$

The fluid and fluid 2 volume averaged equations are shown below.

$$
\begin{aligned}
& c_{p H} \rho_{H}\left\langle m_{H}(x, z)\right\rangle \tilde{\bar{u}}_{H}(x, z) \frac{\partial \tilde{\bar{T}}_{H}(x, z)}{\partial x}+c_{p H} \rho_{H}\left\langle m_{H}(x, z)\right\rangle V_{H}(x, z) \frac{\partial \tilde{\bar{T}}_{H}(x, z)}{\partial z} \\
& =\frac{\partial}{\partial z}\left(\left\langle m_{H}(x, z)\right\rangle\left(\tilde{k}_{t H}(x, z)+k_{H}\right) \frac{\partial \tilde{\bar{T}}_{H}(x, z)}{\partial z}\right)+\tilde{\alpha}_{t H}(x, z) S_{w H}(x, z)\left(T_{S}(x, z)-\tilde{\bar{T}}_{H}(x, z)\right)+\left\langle m_{H}(x, z)\right\rangle \tilde{S}_{T} \\
& c_{p C} \rho_{C}\left\langle m_{C}(x, z)\right\rangle \tilde{\bar{u}}_{C}(x, z) \frac{\partial \tilde{\bar{T}}_{C}(x, z)}{\partial x}+c_{p C} \rho_{C}\left\langle m_{C}(x, z)\right\rangle V_{C}(x, z) \frac{\partial \tilde{\bar{T}}_{C}(x, z)}{\partial z} \\
& =\frac{\partial}{\partial z}\left(\left\langle m_{C}(x, z)\right\rangle\left(\tilde{k}_{t C}(x, z)+k_{C}\right) \frac{\partial \tilde{\bar{T}}_{C}(x, z)}{\partial z}\right)+\tilde{\alpha}_{t C}(x, z) S_{w C}(x, z)\left(T_{S}(x, z)-\tilde{\bar{T}}_{C}(x, z)\right)+\left\langle m_{C}(x, z)\right\rangle \tilde{S}_{T}
\end{aligned}
$$


The equations are solved simultaneously using a forward elimination backwards substitution method. 


\section{Optimization}

Currently, the optimization of heat exchangers is not fully realized. Because of the many different parameters that effect the optimization, a complete optimization incorporating each of those variables is very time consuming. By using VAT, many simulations can be done in a matter of minutes, allowing for optimization, which can require hundreds to thousands of simulations. The VAT code can solve for temperature and velocity distributions of a heat exchanger, and this data can be used to calculate effectiveness or a final cost for each design. These final effectiveness and cost parameters can be used to compare different heat exchanger designs. The relative effect of the parameters for the heat exchanger are shown below in Figure 32, which demonstrates that every variable is important in the optimization process. $\mathrm{A}=$ Air flow, $\mathrm{B}=\mathrm{PD}, \mathrm{C}=$ fin pitch, $\mathrm{D}=$ fin thick, $\mathrm{E}=\mathrm{L}, \mathrm{F}=\mathrm{px} / \mathrm{pd}, \mathrm{G}=\mathrm{NTy}, \mathrm{H}=\mathrm{NTx}, \mathrm{I}=\mathrm{Py} / \mathrm{Pd}$. The other constraints and inputs are discusses in the results section.

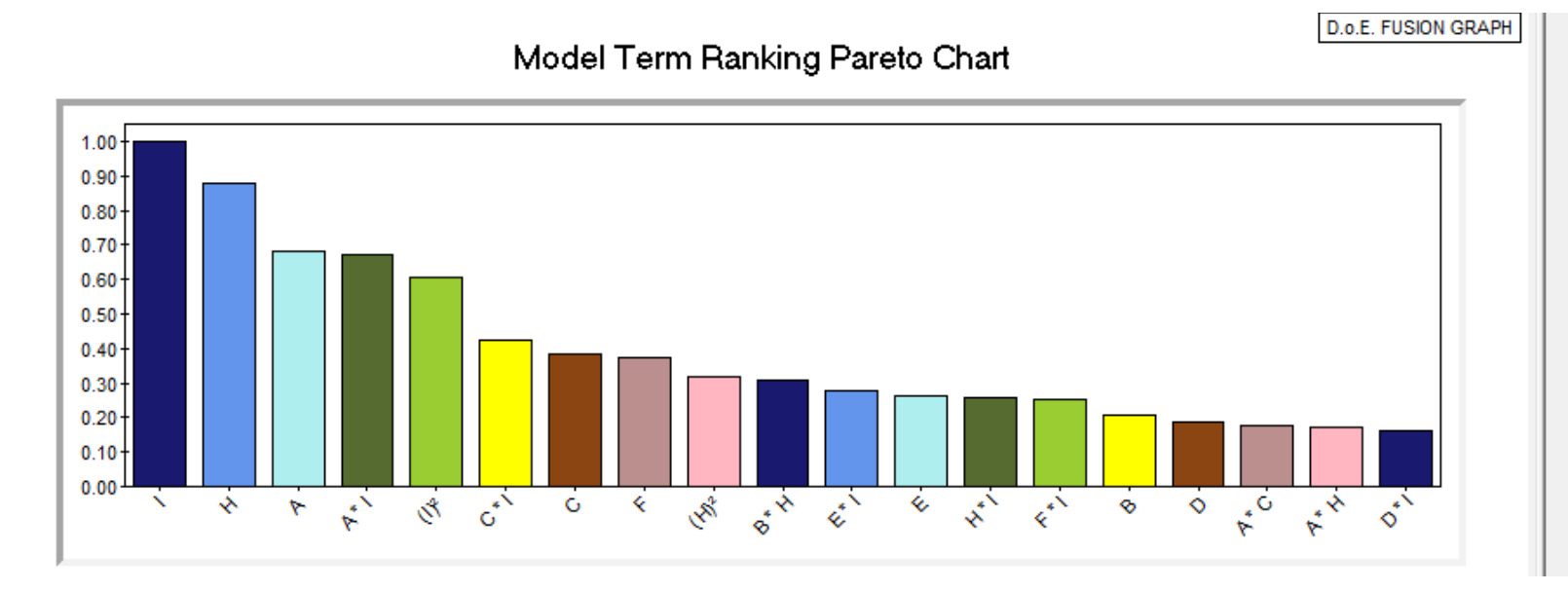

Figure 32: Pareto Chart for HGC

To optimize multi-parameter problems, two main approaches are used: design of experiment (DOE) and genetic algorithm, GA. DOE is based of statistical analysis of data, wherein a 
predetermined number of experiments is done depending on the number and range of parameters to be tested. For example, for 4 parameters, approximately 30 experiments are needed, but for 10 parameters, 100 cases are needed. Once the results for the cases are determined, in this case the cost of each heat exchanger design, a response surface can be made. This response surface is a 3 dimensional surface plot of the effect of one parameter based on the effects of 2 others, while holding the remaining at some fixed value. Using these plots, the user must then visually determine the effect of each parameter on the overall most optimal design. If each parameter has a minimal effect, say 10-20\%, then an optimal design can be found in a few minutes, but if each variable has a large effect, finding the overall optimal design may be difficult if it is not readily clear.

Genetic algorithms are based on the idea of the biological genome, where each generation, a range of possible designs are tested, and the most fit are chosen to remain, then these most fit are either slightly mutated or have their parameters randomly shuffled between them to make a new generation to search for even more optimal designs. Thus, the best heat exchanger design is found through the evolutionary selection of the best fit for the given constraints. Genetic algorithms have a main benefit of not being a visually based method, and thus can be written into the computer code and an optimum selection can be found just by giving the heatsink requirements and given constraints. Even though GAs requires hundreds or thousands of simulations, as it is all done automatically, they have the potential to be faster than DOE.

\subsection{Heat Sink Parameters}

When optimizing the heat exchanger, every parameter that can be varied that has an effect of the design response should be included in the optimization. In the case of a fin tube heat exchanger, these include the geometry of the heat exchanger and the thermal boundary 
conditions or limitations. All these geometry variables is shown in figure below, Tube outer diameter, tube inner diameter, tube pitch in transverse direction tube pitch in longitudinal direction, the fin pitch, and fin thickness. All these geometry variables are used to find the 2 needed variables of the VAT code, the porosity, $\langle\mathrm{m}\rangle$, and surface wetted, $\mathrm{S}_{\mathrm{w}}$. In addition, the weight, values of number of tubes in each direction or overall length, with and height, material of the tubes and fins and 2 fluids are the remaining physical variables. The thermal/flow variables are the flow speed in tubes, flow speed through fins, temperatures of inlet tube and temperature of inlet fin temperature. There are 17 variables in total.

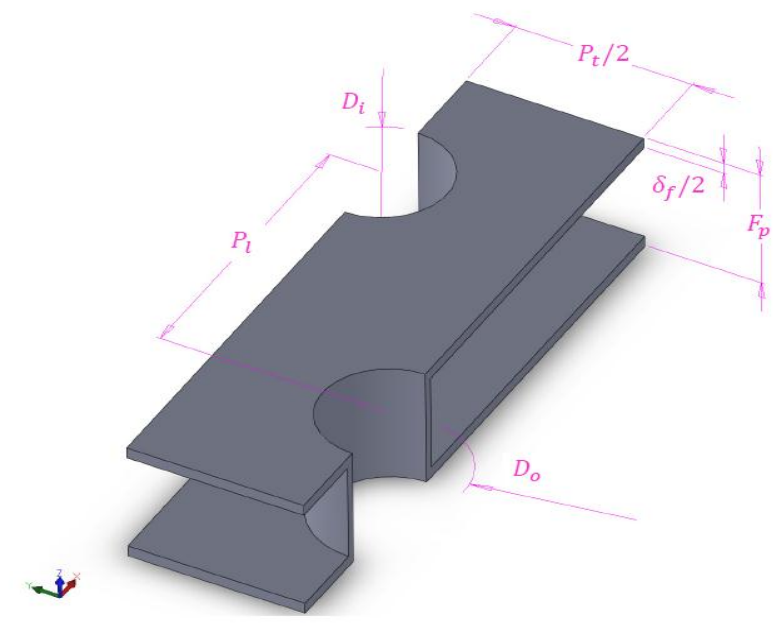

Figure 33: REV with 6 of the geometry parameters

In many cases, only very few of the 17 variables are specified, such as the fluids being air and water, with specific inlet temperatres and with a maximum volume or weight requirement. It is through the use of optimization that the remaining variables are chosen.

In order to evaluate the performance of a ehat exchanger, the heat moved from one fluid to another can be calculated the the changes in each fuid as they flow through the heat exchanger. The other value to be calculated is the pumping power required for the fan on the air side and a pump on the tube side of the heat exchanger. This heat moved and pumping power can be used 
to find the effectiveness of the cost of operation of the heat exchanger. The design with the maximum effectivness or the minimum cost can be chosen.

The value of effectiveness is defined as the heat moved from one fluid to the other per unit volume divided by the pumping power per unit volume per change in temperature

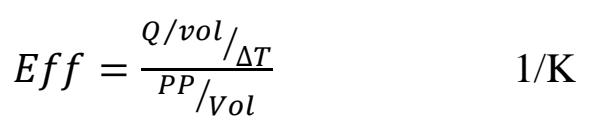

The other value of comparison is the cost of the heat exchanger. One way of defining this is the process used by Orozaliev, et al. 2010 , which is the heat generation cost, the lowest price for heating a fluid.

$$
H G C=\frac{C R F * C E+C_{m n t}+C_{o p}}{Q} \frac{\$}{k W}
$$

With the capital recovery factor $C R F=\frac{i(i+1)^{n}}{(i+1)^{n}-1}$

Capital expenditures, CE, include heat exchanger, fan, pump and piping costs. Fan, pump and piping can all be determined from a local supplier. The $\mathrm{C}_{\mathrm{mnt}}$ is $1 \%$ of $\mathrm{CE}$.

$$
C E=C_{h x}+C_{\text {fan }}+C_{\text {pump }}+C_{\text {pipe }} \$
$$

Operation costs $\mathrm{C}_{\mathrm{op}}$ are calculated by multiplying the annual energy consumption by fan and pump with the cost of electricity. Cost of electricity is $.13 \$ / \mathrm{kwhr}$ and cost of metal was $\$ 5 / \mathrm{kg}$.

$$
\begin{aligned}
& C_{o p}=P P \text { total } * \text { Cost of electricity } \\
& C_{m a t}=\text { Mass } * \text { Cost of metal }
\end{aligned}
$$

The cost of the heat exchanger was found from a correlation fit to the cost data of 74 heat exchanger samples with staggered tube layout varying from small heat exchangers $(1.25 \mathrm{~m} \times 1 \mathrm{~m}$ flow area) to very large ones $(11 \mathrm{~m} \times 2 \mathrm{~m}$ flow area). All samples are from a large manufacturer 
(Güntner Group). Unfortunately, heat exchangers of other producers cannot be used due to the lack of necessary information about geometry and/or prices. This data is shown in Figure 34, below. $\mathrm{C}_{\mathrm{hx}}$ is the cost of a heat exchanger in euro.

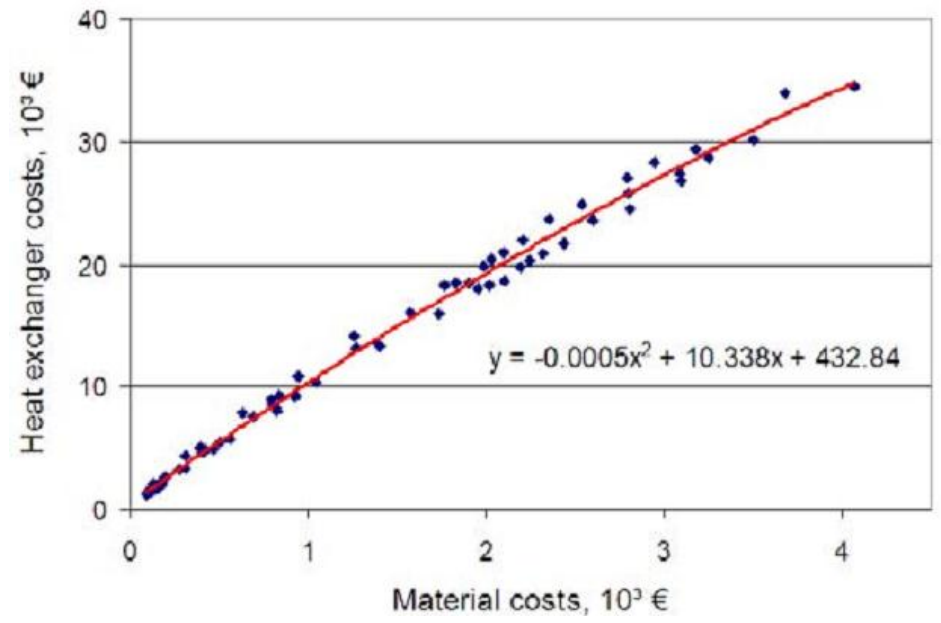

Figure 34: A cost function of the heat exchanger $C_{h x}$ and the source data

$$
C_{h x}=-0.0005 C_{m a t}^{2}+10.338 C_{m a t}+432.84
$$

This cost function, $\mathrm{C}_{\mathrm{hx}}$, allows for the direct comparison of different heat exchanger designs in a real world application where cost is a major factor.

\subsection{Design of Experiment}

The design of experiment currently used uses an initial population of parameters, or the dimensions of the heat exchangers being tested, and fits a response surface to the results of this population. Of course, when first choosing what ranges for the parameters being tested, such as ranging the tube diameter from 1 to $100 \mathrm{~mm}$ will not have enough resolution to find the overall optimal design. The actual optimal may be in the range of $18-24 \mathrm{~mm}$, so many iterations of design ranges may need to be done until the range of parameters chosen gives a good enough resolution for optimization. The first population may find that the optimal diameter may be in the range of $10-40 \mathrm{~mm}$ instead, and a new population should be made with this new range. This 
should be done for each variable in turn, but as each variable has an effect on the others, this process, known as screening, may eliminate some possible heatsink designs that could have meet the requirements. Not only is this process inexact, it is also at the moment manual method. The genetic algorithm can be more easily written into the code such that given the ranges of inputs, one final heatsink design can be output. The results from DOE and genetic algorithm will be compared so that the best optimization method can be used in the code.

The method of optimization of a heat exchanger is shown below in Figure 35.

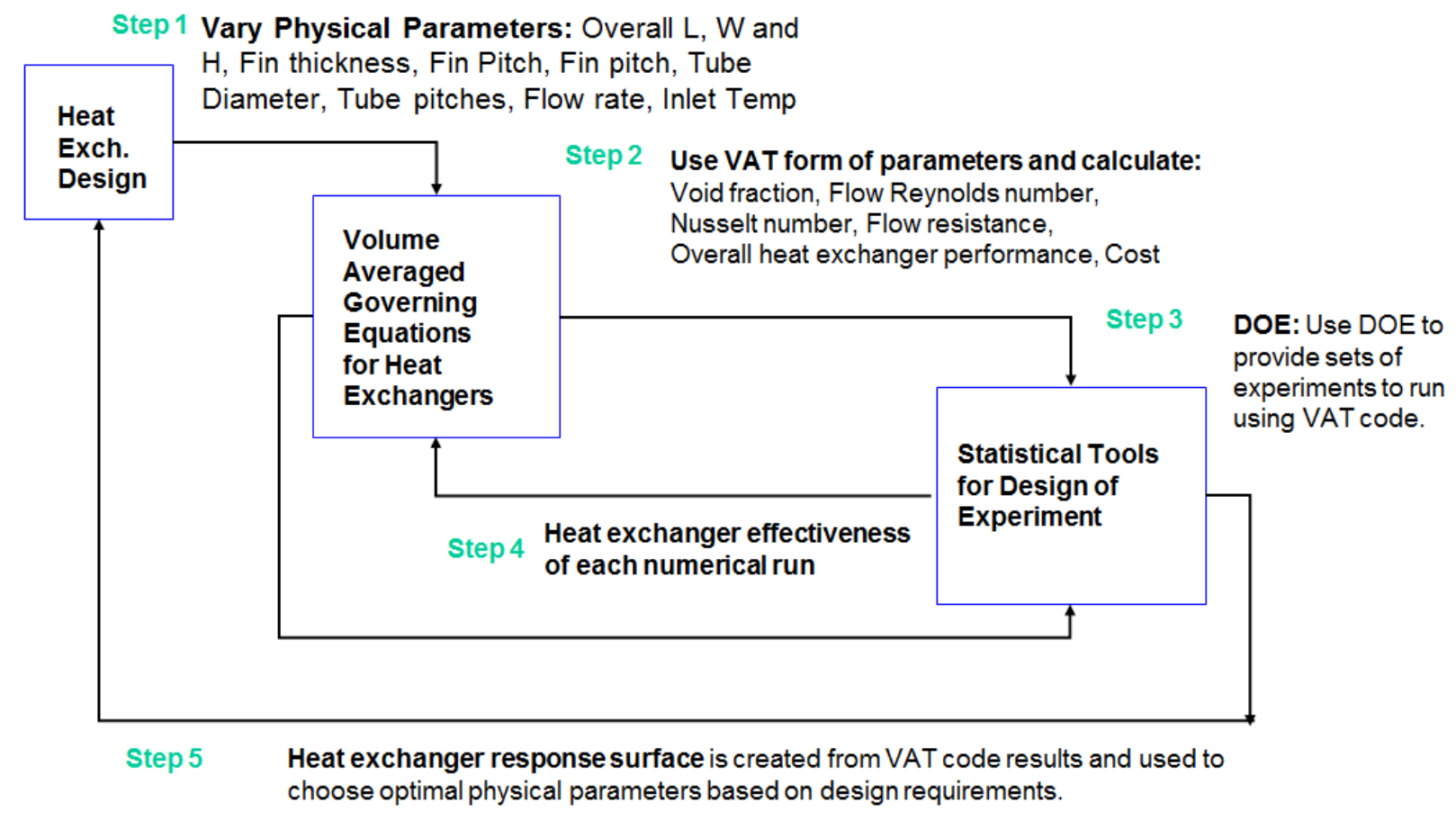

Figure 35: Design of Experiment Flow chart

\subsection{Genetic Algorithm}

The basics of a simple GA are as follows [Goldberg 1989]: An initial population of strings is generated, in our case of heat exchanger optimization; these strings would each be one heat exchanger design. The population would consist of many different designs of heat exchangers, with differing values for each of the parameters listed previously. These strings are evaluated based on a fitness function, 
which is the minimum cost. The cheapest strings that meet the constraints are then selected for mating. During the mating process the strings randomly form groups and exchange their genetic makeup, or geometry dimensions. This process is known as crossover. Subsequently, after random mutations are allowed to take place, a new generation of fitter strings is present, based off the most fit strings from the previous generation. Their fitness's are evaluated and the process repeats. The GA can eventually be combined with other optimization methods to obtain even better results than the GA can give alone, if desired. These could be using a response surface approach on the initial and final populations to verifiy that the GA did not converge on a local optimum but the global optimal design. The flow chart for the genetic algorithm is shown below in Figure 36.

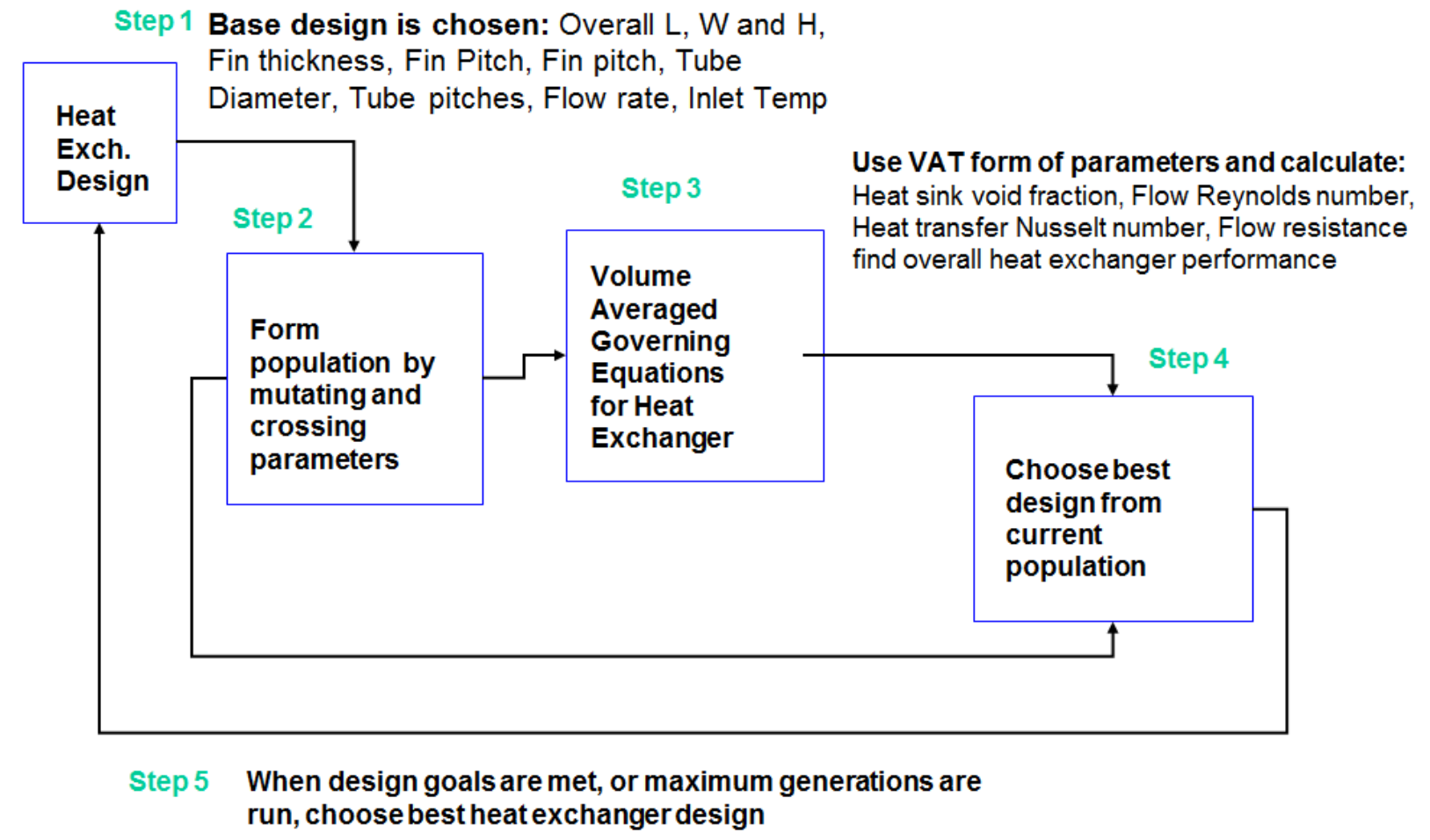

Figure 36: Flow diagram for genetic algorithm 


\section{Results of Optimization}

\subsection{Optimization Parameters}

Using the heat exchanger design from [Figure 31 2010], where a heat exchanger design for a test plant in Bishek [Frank 2006] was optimized and compared one installed at that location which was designed by an established producer. Orozaliev used a model structure is based on the detailed cooling coil model of the ASHRAE4 HVAC Secondary Toolkit implemented in TRNSYS (a flexible tool designed to simulate the transient performance of thermal energy systems). The model accounts for possible condensation of vapor on parts or even all over the heat exchanger surface by considering separately wet and dry parts of the heat exchanger surface. The VAT model developed for this project was used to predict the same cost values as Orozaliev and then optimize using the same range of parameters.

The variation range of some parameters has been restricted by production possibilities (e.g. fin pitch and thickness) or even distribution of the air flow (finned length and height). Tube thickness has not been varied, because the smallest tube thickness will always be selected by the optimization concerning the material costs, pressure drop on the water side and heat conduction in the tube. The smallest tube thickness is determined by the operating pressure. Therefore, the same tube thickness of $0.32 \mathrm{~mm}$ has been specified as in the installed heat exchanger. Other fin (e.g. louvered, split fins) and tube (e.g. oval, flat) types have not been considered because of the absence of respective heat exchanger cost functions.

\begin{tabular}{|l|c|c|c|c|c|c|}
\hline & Unit & Min & Max & Ref & Opt & $\begin{array}{l}\text { VAT } \\
\text { Opt }\end{array}$ \\
\hline T water in & $\mathrm{C}$ & 12 & 12 & 12 & 12 \\
\hline T air in & $\mathrm{C}$ & 35 & 35 & 35 & 35 \\
\hline Water Flow Rate & $\mathrm{kg} / \mathrm{s}$ & 1.66 & 1.66 & 1.66 & 1.66 \\
\hline
\end{tabular}




\begin{tabular}{|l|c|c|c|c|c|c|} 
Air Flow Rate & $\mathrm{m}^{\wedge} 3 / \mathrm{h}$ & 9000 & 40000 & 10000 & 20000 & 15000 \\
\hline Fin Pitch & $\mathrm{mm}$ & 1.5 & 5 & 3 & 1.5 & 3.5 \\
\hline Fin Thick & $\mathrm{mm}$ & 0.15 & 0.45 & 0.25 & 0.15 & 0.15 \\
\hline \# Tubes Y & & 2 & 9 & 6 & 4 & 3 \\
\hline \# Tubes X & & 15 & 90 & 20 & 50 & 55 \\
\hline Px/Pd & & 1.1 & 8 & 4 & 2 & 3 \\
\hline Py/Pd & & 1.2 & 8 & 6 & 3 & 6.5 \\
\hline Tube outer Diameter & $\mathrm{mm}$ & 6 & 22 & 12 & 6 & 8 \\
\hline
\end{tabular}

Table 2: Range of Parameters

As shown Figure 32, every variable has at least a $10 \%$ effect on the outcome of the final cost of the heat exchanger. Every parameter that could be varied should then be included in the optimization process. Once the data becomes available on their respective effects the types of materials used, special effects of the fins (e.g. louvered, split fins) and tube (e.g. oval, flat) types, should all be included in the optimization.

The response parameters used are the cost of the heat exchanger, HGC, the combined pumping power of both the fin side and the tube side in watts, the mass in $\mathrm{kg}$, the volume in $\mathrm{m}^{3}$, QZ2 the heat transferred from the air to the water and the effectiveness is shown below. HTEFF is the ratio of heat moved to the maximum potential heat moved.

$$
E f f=\frac{Q Z 2}{P P T} /_{\Delta T}
$$

\subsection{Results Data}

Design of Experiment (DOE) was performed using Fusion Pro® DOE software (S-Matrix ${ }^{\circledR}$ Corporation). To perform the optimization, the 9 parameters listed above were given to the program, and Fusion Pro produced 106 different heat exchanger designs to evaluate using the VAT code. The VAT code was used to find the response parameters listed above, which were set 
to the values shown below in Table 3. The values of HGC, EFF and QZ2 were all maximized while Volume, Mass and Pumping Power were minimized. The goal parameters represent the line on the following results figures. The white region on each plot is the area where all 6 parameters have their goals met. The goals could be changed so that the white areas became smaller, then the variable parameters could be adjusted so that they result in the maximum possible white area. This is repeated until the optimal design is found. Any combination of design parameters that result in the white are would create and acceptable heat exchanger.

\begin{tabular}{|l|l|r|r|r|r|}
\hline Parameter & Unit & Goal & Ref & Opt & VAT \\
\hline HGC & Euro/Kw & 0.007 & 0.0075 & 0.0053 & 0.005 \\
\hline EFF & $1 / \mathrm{K}$ & 2 & 0.245 & 0.45 & 2 \\
\hline Volume & $\mathrm{m}^{\wedge} 3$ & 0.5 & 0.229 & 0.199 & 0.25 \\
\hline Mass & $\mathrm{kg}$ & 50 & 44.3 & 19.46 & 27 \\
\hline Pumping Power & $\mathrm{w}$ & 500 & 1200 & 1760 & 1000 \\
\hline Qz2 & $\mathrm{Kw}$ & 70000 & 82000 & 132000 & 80000 \\
\hline HTEFF & $*$ & 0.45 & 0.25 & 0.45 & 0.51 \\
\hline
\end{tabular}

Table 3: Results parameters 

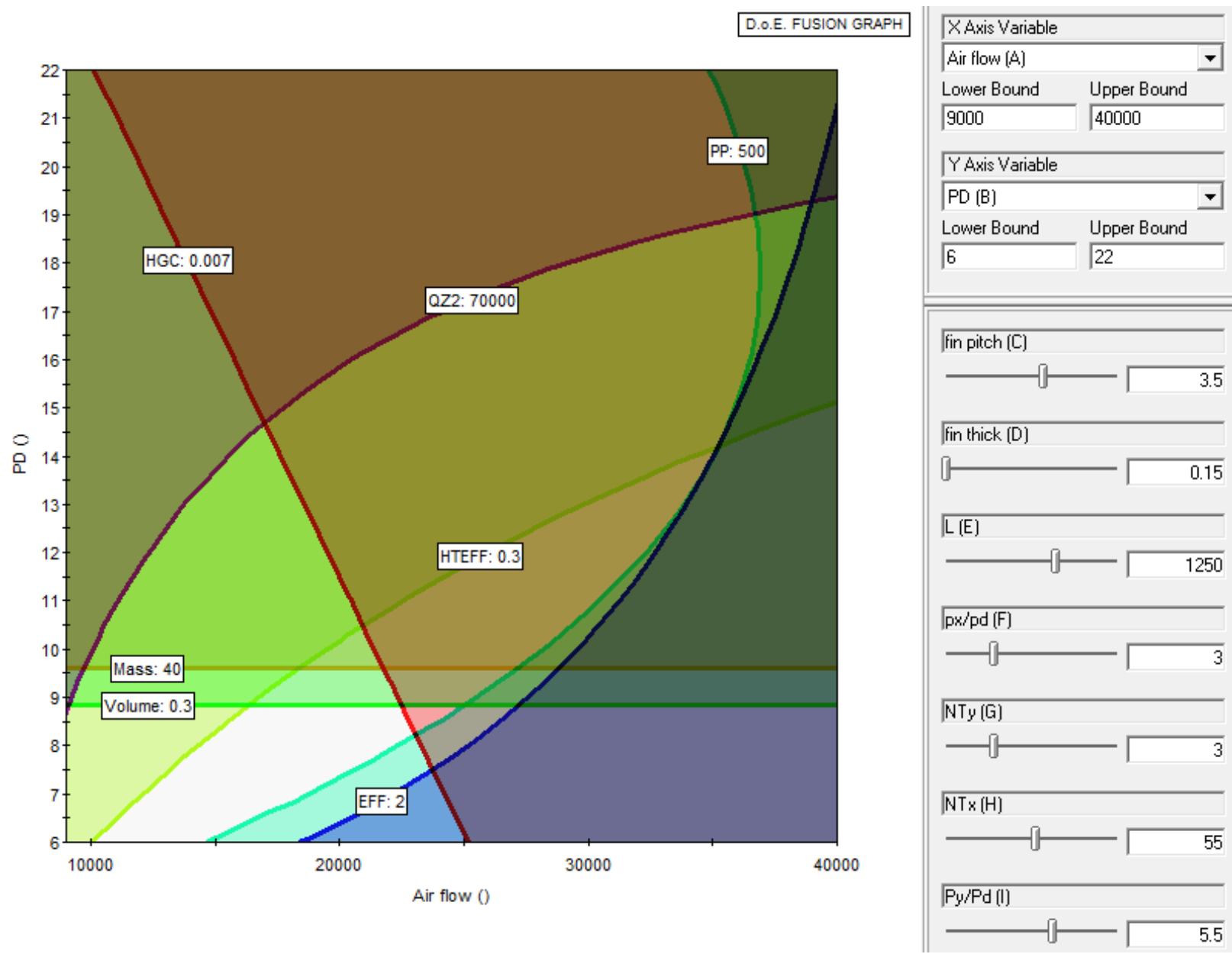

Figure 37: The effect of flow rate versus tube diameter

Figure 37 shows the effect of varying air flow and tube diameter while keeping the other parameters constant. The values of the other parameters are shown in the figure. From this figure, the effects of the airflow and tube diameter can be found. The airflow is optimal around the value of $15000 \mathrm{~m}^{\wedge} 3 / \mathrm{hr}$ and the tube diameter is found to be optimal around $8 \mathrm{~mm}$. The value of airflow is found as the balance between increasing the value of heat moved, Q and the HTEFF and decreasing the pumping power. Increasing the value of the tube diameter will decrease the Q, pumping power and effectiveness. 

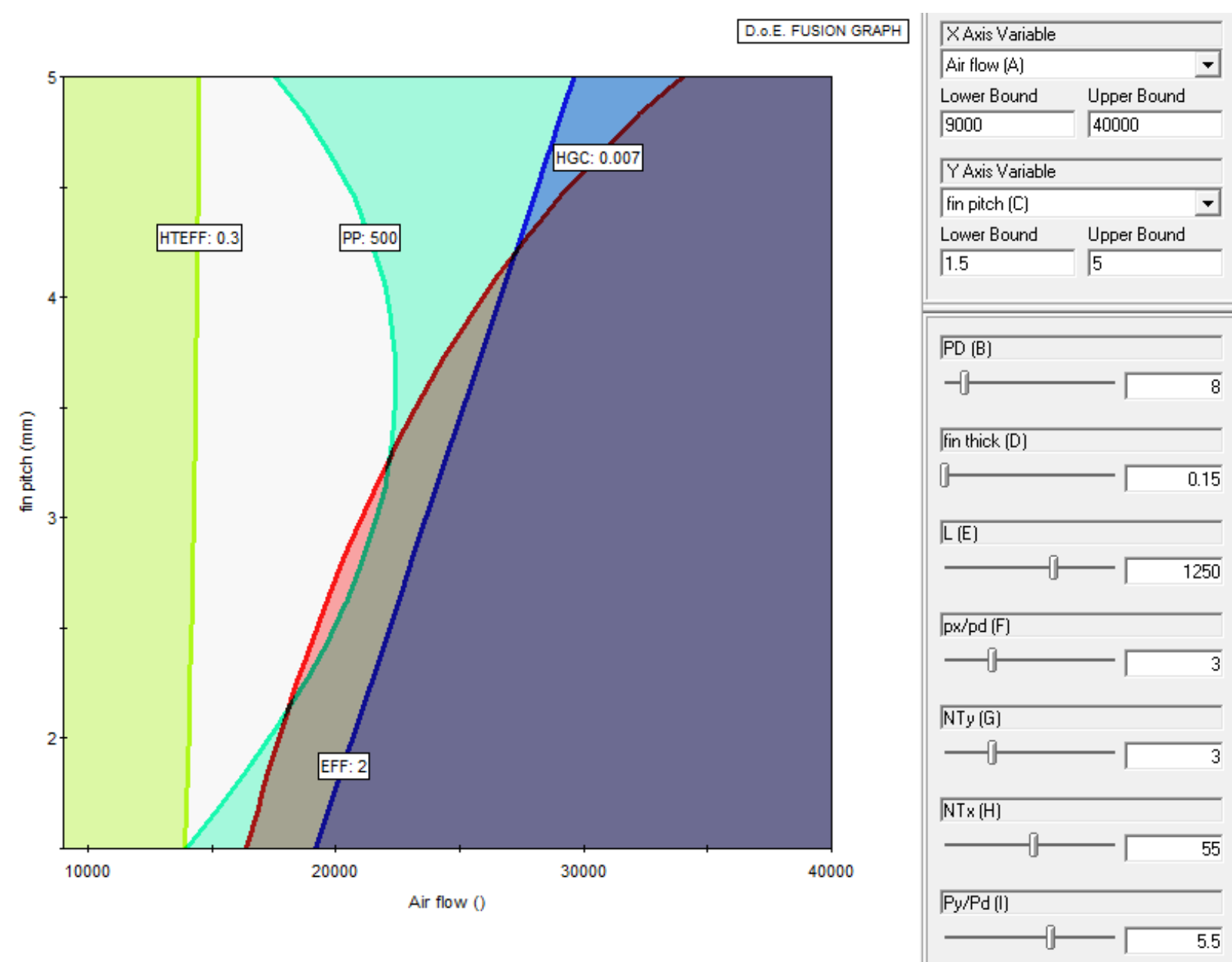

Figure 38: Flow rate versus Fin pitch

Figure 38 shows the effect of the flow rate versus the fin pitch. The fin pitch has the effect of opening up the airside of the heat exchanger, so increasing it reduces the pumping power and increases the effectiveness. The optimal value is found in the middle of the range, allowing for the maximum pumping power. 

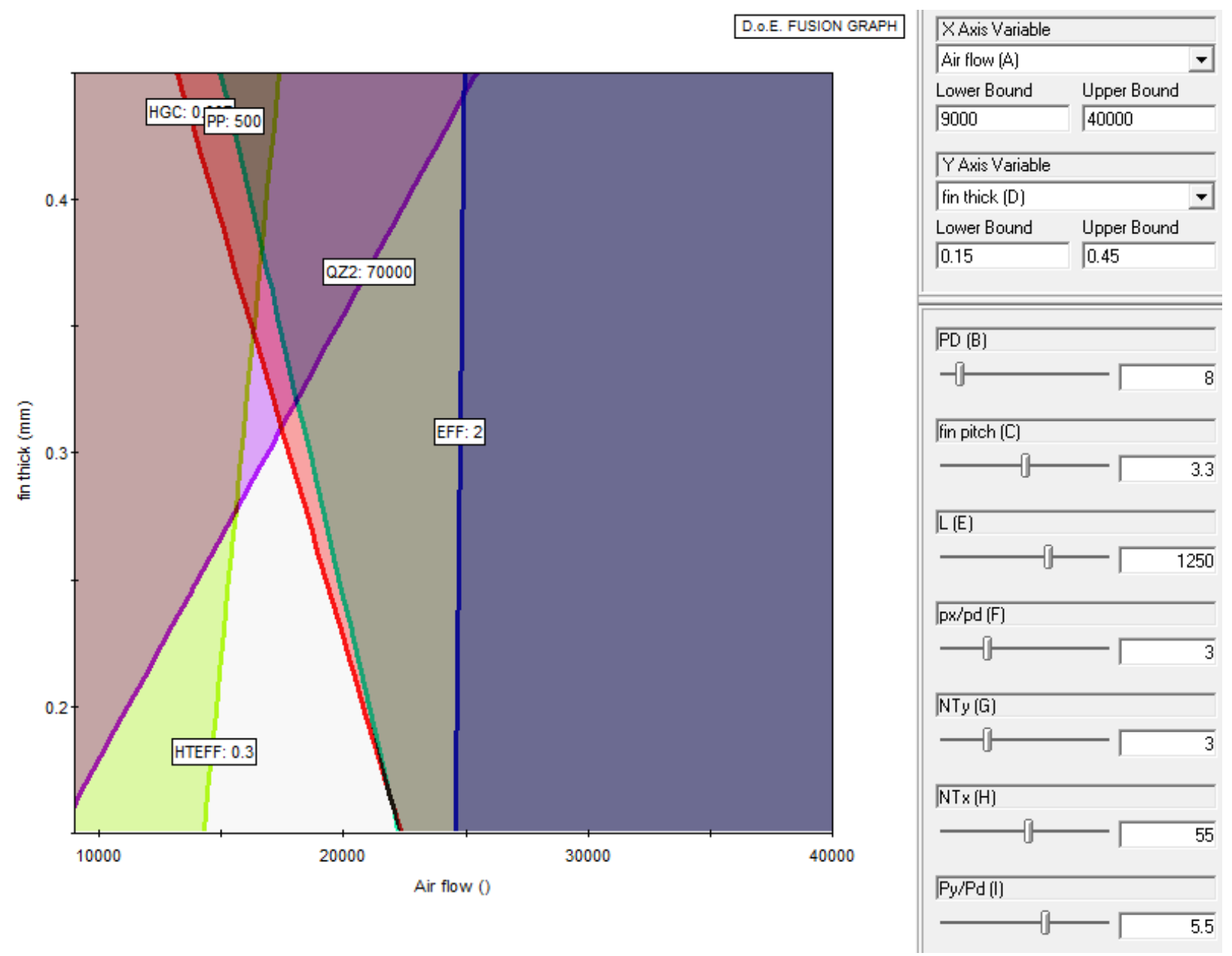

Figure 39: effect of flow rate versus fin thickness

Figure 39 shows the effect of flow rate versus fin pitch. The fin thickness will increase the conductance of the heat exchanger; with increasing fin thickness increases the cost, pumping power and Q, but decreases the effectiveness. The optimal value would be in the lower limit, and if the fin thickness would be made any thinner than the limit of $0.15 \mathrm{~mm}$ that was imposed because of manufacturing limits, it should be chosen. 

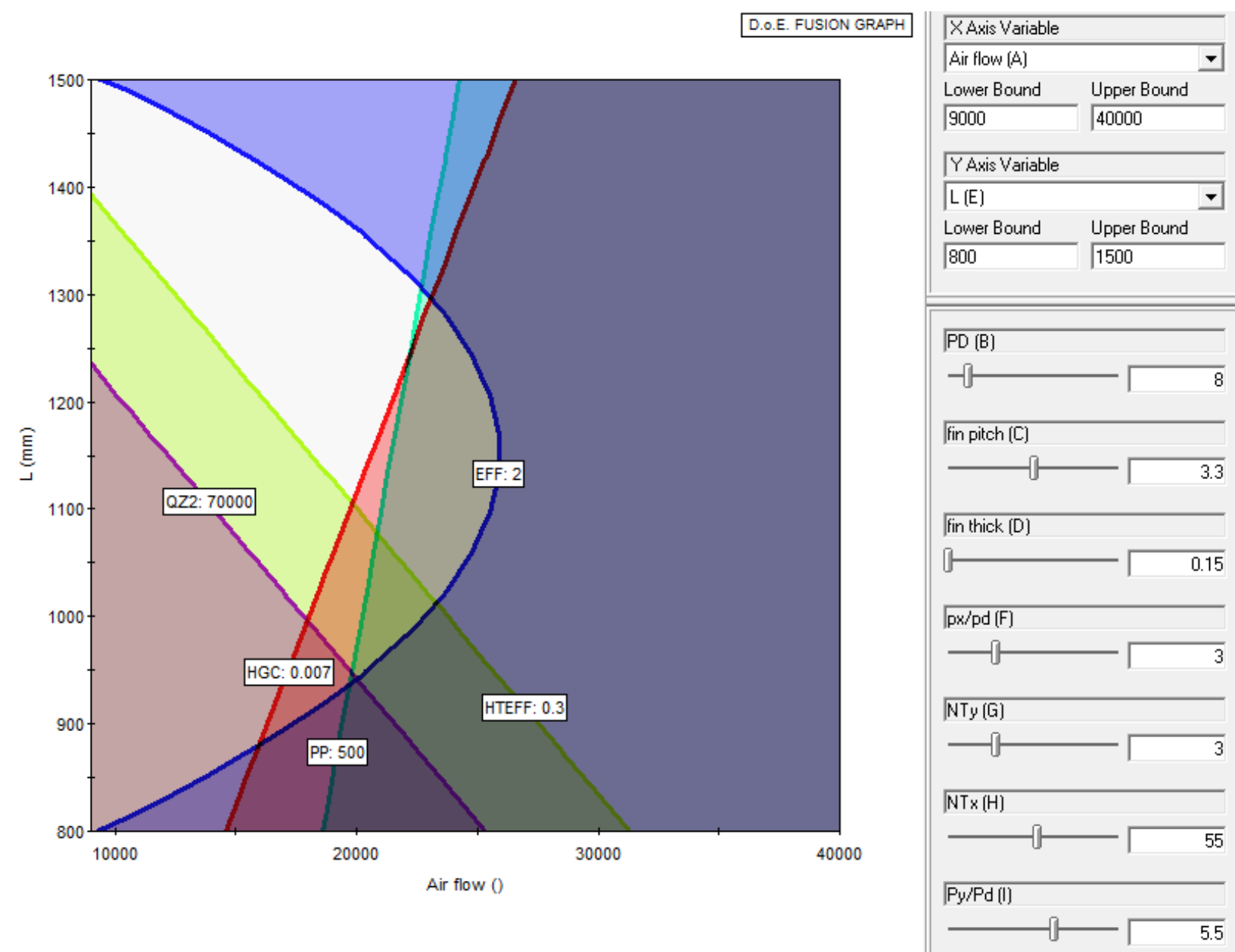

Figure 40: Effect of length versus air flow rate

Figure 40 shows the effect of the finned length versus the airflow rate. The finned length increases the area of the heat exchanger and thus the volume and weight, but also the heat moved through and the HTEFF. As increasing the length also reduces the pumping power yet at the same time reduces the cost, the effectiveness peaks in the middle of the range of length, and thus a value in the mid range is chosen, of $1250 \mathrm{~mm}$. 

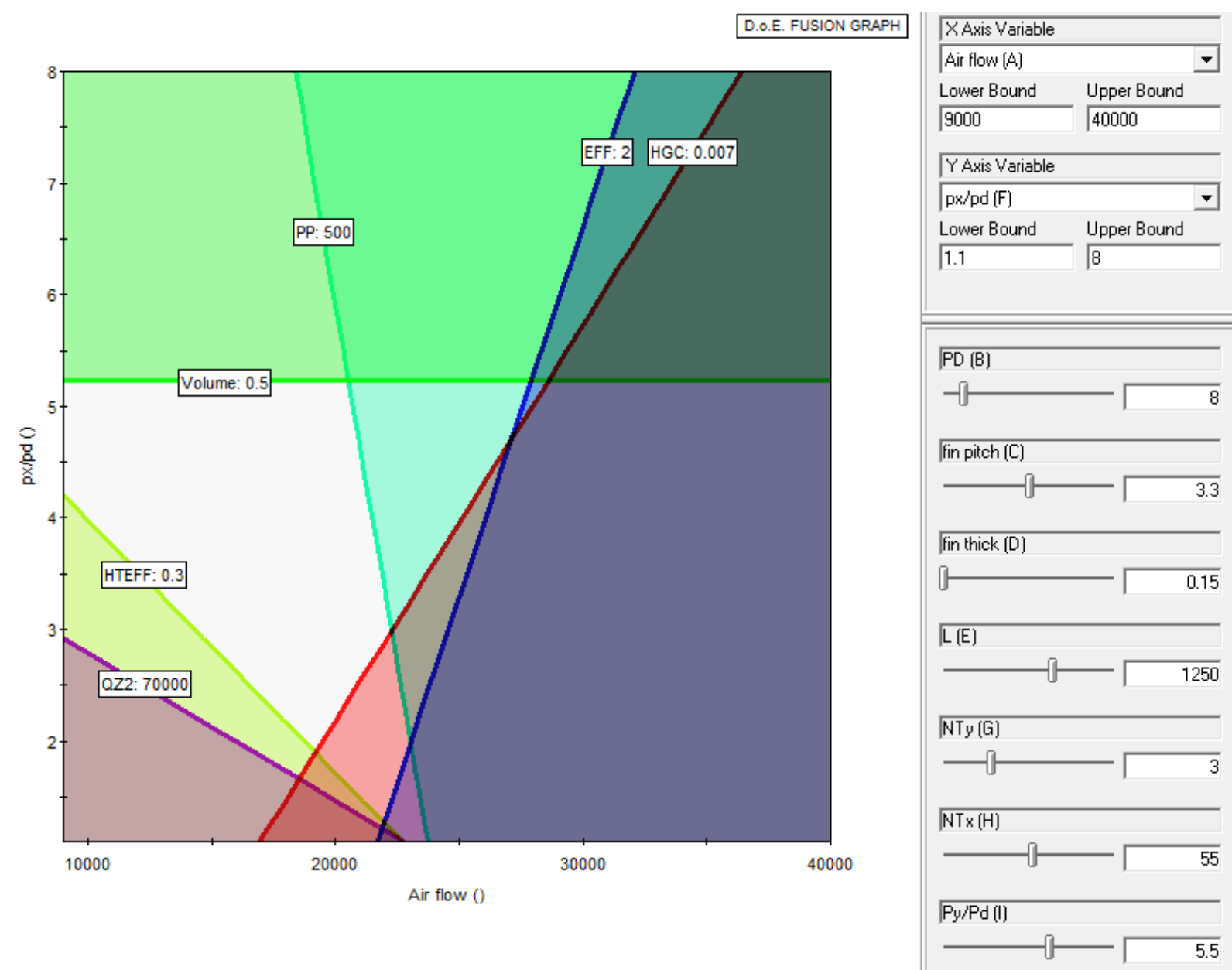

Figure 41: Effect of Tube pitch in the $x$ direction versus air flow

Figure 41 shows the effect of tube pitch in the $\mathrm{x}$ direction versus the air flow rate. Increasing tube pitch in $\mathrm{x}$ direction increases the heat moved, volume, and effectiveness, but decreases cost. However, increasing pitch also increases the size of the heatsink, the reduced pressure drop would help the flow up to a point and then the larger flow area would result in larger cost, so the optimal level of pitch in the $\mathrm{x}$ direction is a ratio of 3 to the tube diameter. 

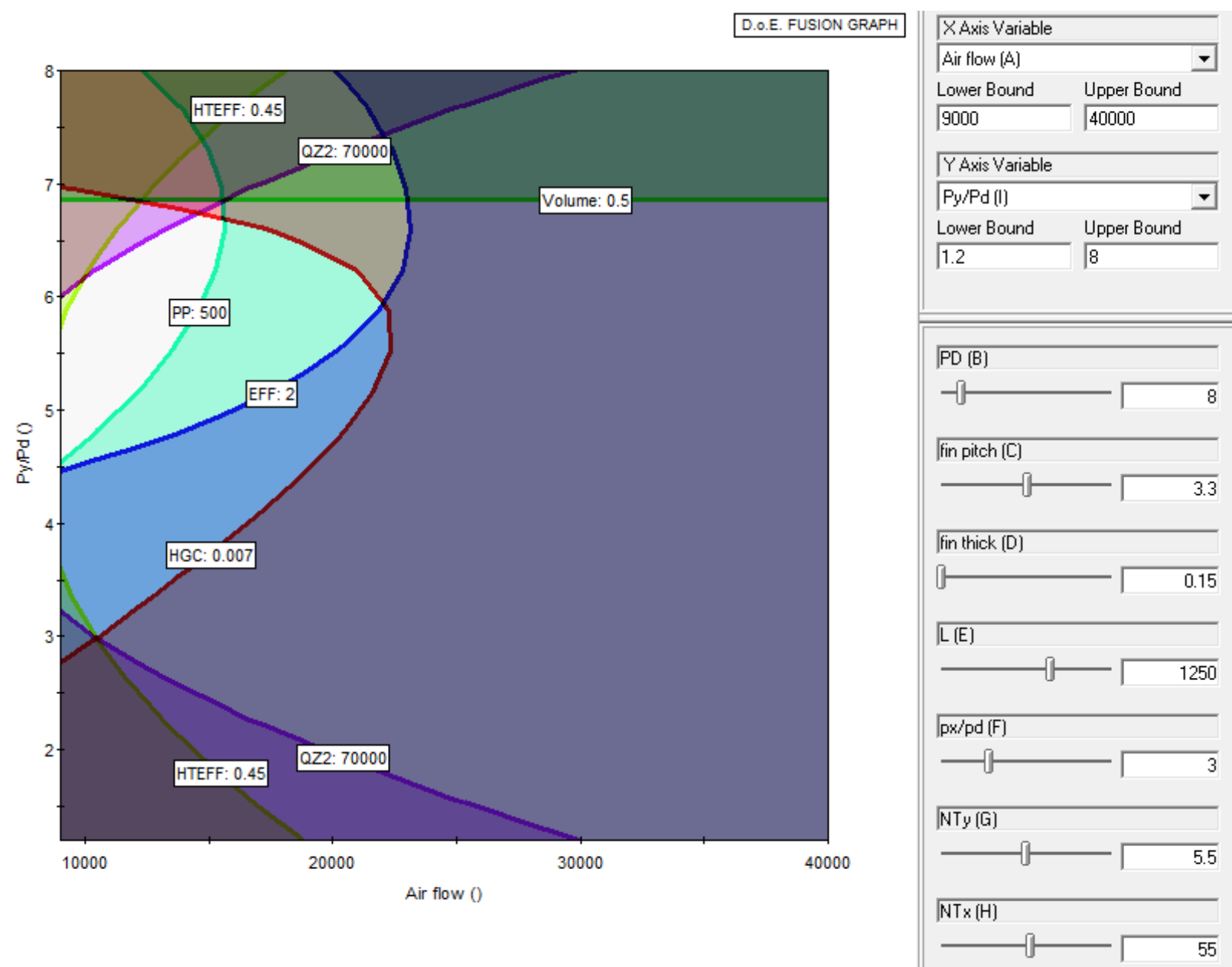

Figure 42- Effect pitch of tubes in y direction versus air flow.

Figure 42 shows the effect of pitch in the y direction versus air flow on the performance of the heat exchanger. It should be noted in this figure that the HTEFF goal was raised to 0.45, a much higher value than the previous entries. The optimal value of the pitch of the tubes in the $y$ direction is roughtly 6.5 times the tube diameter. This can be seen as increasing or decreasing the value would result in a higher cost and pumping power, with a lower value or heat moved. 


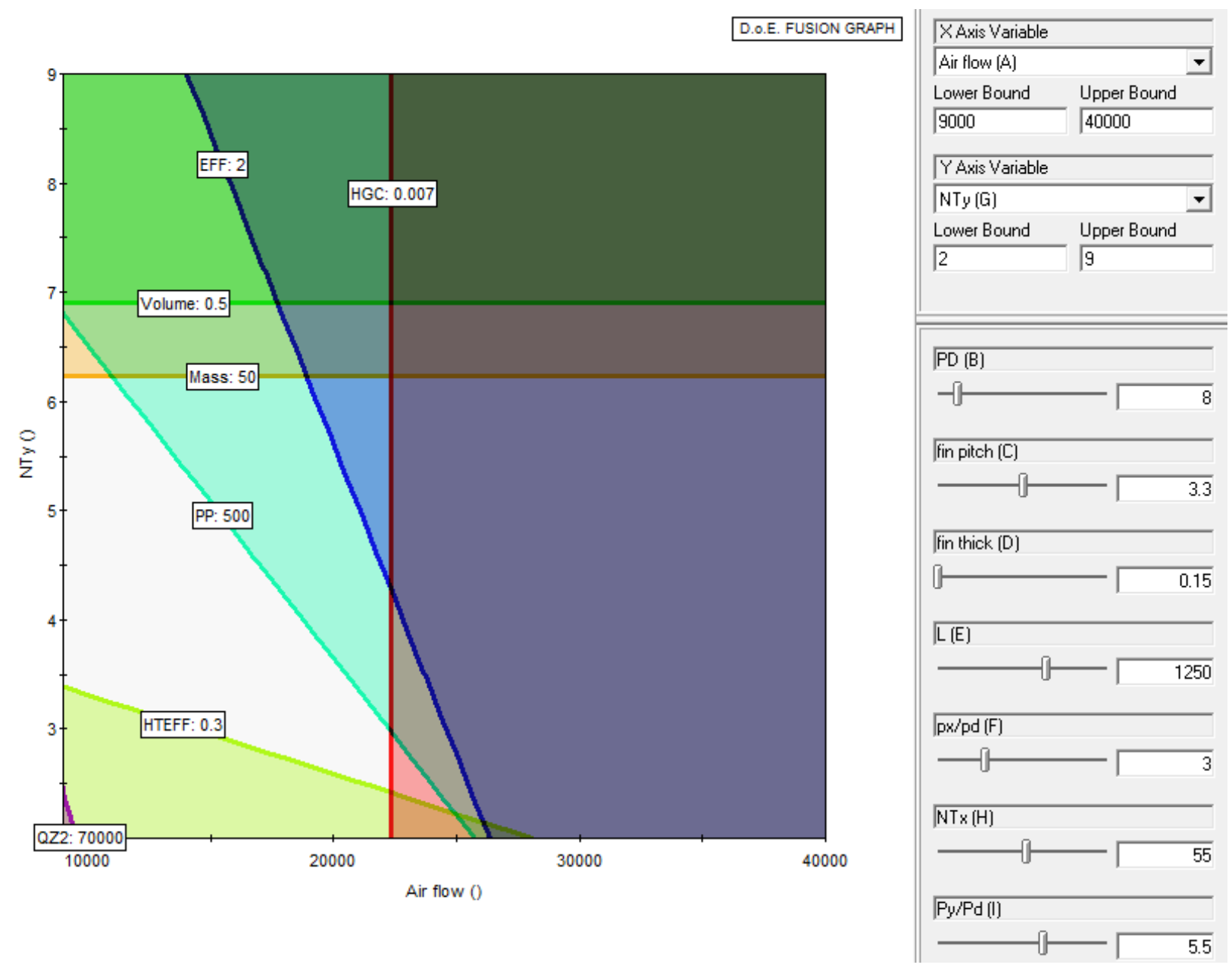

Figure 43: Effect of number of tubes in y direction versus airflow.

Figure 43 shows the effect of the number of tubes in the y direction versus the airflow rate. Increasing the number of tubes in the y direction increases the flow length and thus pressure drop, volume and mass, heat moves and HTEFF. However, it dreceases the effectiveness, yet has minimal effect on the cost. The optimal value is chosen to be 3 tubes. 


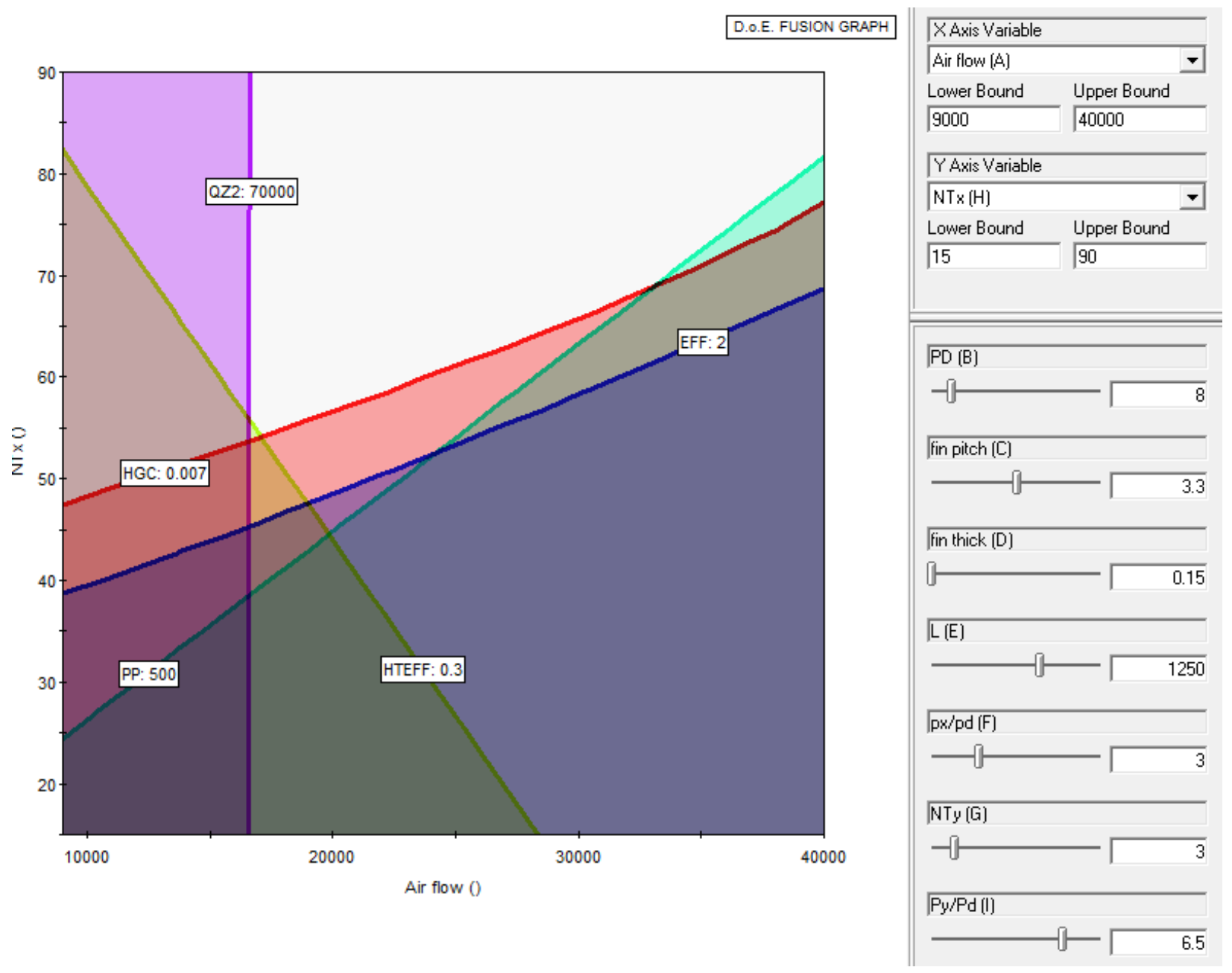

Figure 44: Effect of number of tubes in the $x$ direction versus the air flow rate

Figure 44 shows the effect of the number of tubes in the $\mathrm{x}$ direction versus the air flow rate. The optimal value for the number of tubes in the $\mathrm{x}$ direction is at the intersection of the flow rate and cost goals, being 55 tubes.

\subsection{Discussion of Results}

The VAT code predicts a much more efficient heat exchanger than the results from Orozaliev's method. This could have arisen from many factors, including Orozaliev's use of an annual cycle to compare heat exchangers, which would have incorporated annual temperature and humidity data for ambient air. Orozaliev's model also incorporated 2 phase effects and a 
more in depth pump/fan cost analysis. However, both methods compare well in the final pricing scheme and more experimental data is needed to fully validate and compare both methods. The optimal design of thinest fins, the number of tubes and flow rates compares well between both optimums. 


\section{Project Publications, Presentations and Technology Transfer}

Catton, Ivan, "Heatsink Design and Optimization", Proceedings of the 14th International Workshop on Thermal investigations of ICs and Systems (THERMINIC), Rome, Italy, 24-26 Sept 2008

An increasing demand for a higher heat flux removal capability within a smaller volume for high power electronics led us to focus on micro channels in contrast to the classical heat fin design. A micro channel can have various shapes to enhance heat transfer, but the shape that will lead to a higher heat flux removal with a moderate pumping power needs to be determined. The micro channel geometries explored are pin fins (staggered) and parallel plates. The problem solved here is a conjugate problem involving two heat transfer mechanisms; 1) porous media effective conductivity and 2) internal convective heat transfer coefficient. Volume Averaging Theory (VAT) is used to rigorously cast the point wise conservation of energy, momentum and mass equations into a form that represents the thermal and hydraulic properties of the micro channel (porous media) morphology. Using the resulting VAT based field equations, optimization of a micro channel heated from one side is used to determine the optimum micro channel morphology. A standard commercial size and design is chosen for analysis and to demonstrate the utility of the VAT based process.

Tam, V.K., Chang, M.R., and Catton, I. "The use of volume averageing theory (VAT) for evaluation of surface heat transfer augmentation". Proceedings of IMECE 2008, ASME IMECE2008-68194 (2008), Boston, MA.

A model, based on Volume Averaging Theory (VAT), was developed to predict friction and heat transfer over augmented surfaces. The objective is to find augmentation that produces a higher heat removal relative to the increase in pumping power necessary to overcome the additional friction that is generated. This was accomplished with a numerical solution of the VAT transport equations for describing fully developed turbulent flow in a channel with augmented surfaces. Friction factor and Nusselt number results produced by the numerical simulation are compared with experimental data to validate the model and which of the augmented surfaces performs the best is clearly shown.

Vadnjal, A., and Catton, I. "A general approach to analysis of flow and heat transfer in packed beds". Proceedings of IMECE 2008, ASME IMECE2008-68189 (2008), Boston, MA.

It is postulated that proper scaling will collapse the multiplicity of data for friction and heat transfer coefficient to a usable reasonably general formulation by choosing the hydraulic diameter as $\mathrm{Dh}=4<\mathrm{m}>/ \mathrm{Sw}$. The chosen hydraulic diameter allows the transformation and comparison of correlation equations and experimental results obtained for diverse media morphologies. Also, it allows experimentally-determined characteristics of the media to be related to the closure relationship derived from the VAT analysis. The numerical results of closure are presented and are compared to various experimental results. The Nusselt number is based on the media internal local surface average transfer coefficient and the friction factor is the local internal value. Results obtained by VAT closure using direct numerical simulation show reasonable agreement between calculated local friction factors and local heat transfer coefficients and data confirming that the friction factor and heat transfer coefficient when correctly scaled can be computed numerically with satisfactory results. 
Hansen, N. and Catton, I. "Multi-Level Heatsink Optimization", Proceedings of the ASME Summer Heat Transfer Conference, ICONE14, ASME HT2009-88293, July 19-23, 2009, San Francisco, CA, USA

Closure for the upper level is obtained using VAT to describe the lower level and a simple plane fin with inner surface augmentation is chosen to optimize. The lower level augmentation chosen is an in line array of dimples with two parameters, pitch and diameter, needed for their description, along with a scale roughened surface with either forward or ownwardd scales. The mid-level description requires fin pitch, base and tip thickness, shape, and height and the upper-level requires overall heatsink height, width and length, and baseplate thickness. A common size heatsink (4 inch width, 4 inch length and 1 inch overall height) with a required heat removal of one kilowatt, an inlet temperature of $30 \mathrm{OC}$, and a maximum allowable center temperature of $650 \mathrm{C}$ is used to demonstrate an approach to heatsink optimization. This is an 8 parameter problem. Significant improvement in performance (heat removal/ pumping power) and reduction in thermal resistance are shown to be achievable.

\section{Catton, I. "CONJUGATE HEAT TRANSFER WITHIN A HETEROGENEOUS}

HIERARCHICAL STRUCTURE". International Heat Transfer Conference, August 8-13, 2010, Washington D.C., USA . IHTC14-23421.

The transport equations are shown to have additional integral and differential terms. The description of the structural morphology determines the importance of these terms and the range of application of the closure schemes. A natural way to transfer from transport equations in a porous media with integral terms to differential equations with coefficients that could be experimentally or numerically evaluated and determined is described. The relationship between CFD, experiment and closure needed for the volume averaged equations is discussed. Mathematical models for modeling momentum and heat transport based on well established averaging theorems are developed. Use of a 'porous media' length scale is shown to be very beneficial in collapsing complex data onto a single curve yielding simple heat transfer and friction factor correlations. The general transport equations developed for a single phase fluid in a heat exchange medium have many more integral and differential terms than the homogenized or classical continuum mechanics equations. Once these terms are dealt with by closure, the resulting equation set is relatively simple and their solution is obtained using simple numerical methods quickly enough for multiple parameter optimizations using Design of Experiment (DOE) or Genetic Algorithms (GA). Current efforts to significantly improve the performance of a HE for electronic cooling, a two temperature problem, and of a finned tube heat exchanger, a three temperature problem, are described.

Hansen, N., Zhou, F., and Catton, I. "Heat Sink Optimization; A Multi-Parameter Optimization Problem". International Heat Transfer Conference, August 8-13, 2010, Washington D.C., USA . IHTC14-22968.

Earlier efforts to optimize a simple pin fin heat sink only considered in line or rotated square configurations, a six or seven parameter problem. In this work, a staggered non-square array, two pitch dimensions, with non-circular pin cross-section are added to the heat sink description. The effects of optimizing fin eccentricity and pitch have both been shown to 
enhance the convective heat transfer as well as to increase the required pumping power thereby contributing to the performance. With a VAT based model, multiple heat sinks can be analyzed quickly while being able to change all dimensions. Using commercial software, S-Matrix Corporation's DOE Fusion, a multi-parameter pin fin heat sink is optimized.

Zhou, F., Hansen, N. and Catton, I. "Obtaining Closure for Heat Exchanger Modeling Based Volume Averaging Theory (VAT)". International Heat Transfer Conference, August 8-13, 2010, Washington D.C., USA. IHTC12-22971.

Volume Averaging Theory (VAT) has been used to rigorously cast the point-wise conservation of energy, momentum and mass equations into a form that represents the thermal and hydraulic properties of heat exchanger channel morphology. At the lower level, the media is described by a representative elementary volume (REV). Closure terms in the VAT equations are related to a local friction factor and a heat transfer coefficient of the REV. The terms in the closure expressions are complex and are evaluated using scaling suggested by VAT from either experimental data or the output of a CFD code. The VAT equations for a finned tube heat exchanger are given and that the key parameters can be obtained by suitable scaling is demonstrated.

David J. Geb, Ivan Catton "Non-intrusive Heat Transfer Coefficient Determination in a Packed Bed of Spheres" International Heat Transfer Conference, August 8-13, 2010, Washington D.C., USA IHTC14-22354

Non-intrusive measurements of the internal average heat transfer coefficient in a randomly packed bed of spherical particles are made. It is desired to establish accurate results for this simple geometry so that the method used can then be extended to determine the heat transfer characteristics in any porous medium, such as a compact heat exchanger. Under steady, one-dimensional flow the spherical particles are subjected to a step change in volumetric heat generation rate via induction heating. The average heat transfer coefficient is determined by comparing the results of a numerical simulation based on volume averaging theory with the experimental results. More specifically, the average heat transfer coefficient is adjusted within the computational procedure until the predicted values of the fluid outlet temperature match the experimental values. The only information needed is the basic material properties, the flow rate, and the experimental data. The computational procedure alleviates the need for solid and fluid phase temperature measurements, which are difficult to make and can disturb the solid-fluid interaction. The success of this method, in determining the average heat transfer coefficient in a randomly packed bed of spheres, suggests that it can be used to determine the average heat transfer coefficient in other porous media.

Feng Zhou Nicholas Hansen and Ivan Catton, "NUMERICAL STUDY OF PLATE-PIN FIN HEAT SINKS WITH VARIOUS PIN CROSS-SECTION PROFILES” ASME 2010

International Mechanical Engineering Congress \& Exposition. IMECE2010-39613

The plate-pin fin heat sink (PPFHS) is composed of a plate fin heat sink (PFHS) and some pin fins planted between the flow channels. In this paper, a numerical investigation was performed to compare the thermal and hydraulic performances of the PPFHSs and PFHS. PPFHSs with five forms of pin ross-section profiles (square, circular, elliptic, NACA 0050, and dropform) were 
numerically simulated. The influence of pin fin cross-section profile on the flow and heat transfer characteristics was presented by means of Nusselt number and pressure drop. It is found that the Nu number of a PPFHS is at least 35\% higher than that of a PFHS used to construct the PPFHS at the same Reynolds number. Planting circular and square pins into the flow channel of heat sinks enhances the heat transfer at the expense of high pressure loss. Using the streamline shaped pins, not only the pressure drop of the compound heat sinks could be decreased considerably, the heat transfer enhancement also makes a step forward. A comprehensive performance comparison is conducted to make a synthetical comparison of PPFHSs and PFHS. It is revealed that the elliptic-pin and NACA-pin PPFHSs have the best overall performance. The heat transfer enhancement of square PPFHS is offset by its high pressure drop, which make it not as efficient as the other types of PPFHS. Circular PPFHS performs similar to the streamline shaped PPFHS when the Reynolds number is not too high. However, with the increase in Re the advantage of the circular cross-section diminishes. The present numerical simulation provides original information of the influence of different pin-fin cross-section profiles on the thermal and hydraulic performance of the new type compound heat sink, which is helpful in the design of heat sinks.

Feng Zhou Nicholas Hansen and Ivan Catton, "DETERMINING THE COMPUTATIONAL DOMAIN LENGTH TO OBTAIN CLOSURE FOR VAT BASED MODELING BY 3D NUMERICAL SIMULATION AND FIELD SYNERGY ANALYSIS” ASME 2010 International Mechanical Engineering Congress \& Exposition. IMECE2010-37561

To get reasonable lower scale flow field and heat transfer solutions, the length of computational domain must be determined in advance. There-dimensional numerical simulations for laminar heat transfer and fluid flow characteristics of plain finned tube heat exchangers were performed. The effects of two factors,

Reynolds number and tube row number were examined. The Reynolds number based on the fin collar outside diameter varied from 500 to 6000 and the corresponding air frontal velocity was ranged from $0.38 \mathrm{~m} / \mathrm{s}$ to $4.6 \mathrm{~m} / \mathrm{s}$. The cases with tube row number varying from 1 to 9 were tested numerically. Field synergy principle analysis was performed for the results, including the indepth analysis of every REV, which gave a clear perspective of the variation of heat transfer performance with the tube rows. It is found that when the number of tube row $\mathrm{N}>4$, the increasing trend of the intersection angle decreases and almost keep constant when $\mathrm{N}>6$, which leads to the heat transfer approaching fully developed conditions.

Simulations over the computational domain with a length of 5+2+2 REVs were recommended to obtain a reasonable local flow and heat transfer field, and then the VAT based closure formulas for drag resistance coefficient and heat transfer coefficient were integrated over the sixth and seventh REV to close the heat exchanger modeling based volume averaging theory.

Feng Zhou Nicholas Hansen and Ivan Catton, "VAT BASED MODELING OF HEAT EXCHANGER AND OBTAINING CLOSURE FROM CFD SOLUTION" ASME 2010 International Mechanical Engineering Congress \& Exposition. IMECE2010-37519

In this work we use CFD to obtain detailed solutions to flow through an element of a heat exchanger and use these results to evaluate the closure terms needed for a fast running VAT 
based code The VAT based code can then be used to solve the heat transfer characteristics of the higher level heat exchanger. A comparison is then made of the CFD closure and experimental data rescaled by VAT scaling. The objective is to show how heat exchangers can be modeled as porous media based on Volume Averaging

Theory and how CFD can be used in place of a detailed, often formidable, experimental effort. 


\section{Conclusion and Future Work}

An optimization procedure of the heat exchanger design is described and shown on a nonstandard application. The target function for the optimization is the cost effectiveness. In the optimization, different heat exchanger geometry and operating parameters have been varied by a design of experiment method. The influence of the heat exchanger design on its costs, heat transfer and friction performance has been evaluated economically by determining investment and operation costs of the system. For the investigated application, an optimized heat exchanger design leads to lower heat generation (transfer) costs compared to the reference configuration. Such a large optimization potential is definitely to some extend caused by special boundary conditions of the non- standard application and by the lack of experience of the design engineer with these constrains. A critical point in the optimization procedure is the relatively high uncertainty of the heat exchanger model and cost functions. In order to increase the accuracy and reliability of the optimization results, more precise heat exchanger models and cost functions are necessary.

In a heat transfer problem, the heat transfer coefficient defined locally was shown to be very effective for two reasons. First, it is independent of the type of heating or boundary condition. Second, it was shown that the local heat transfer coefficient is constant throughout the domain and that it is independent of the entrance length. By using CFD and experiment to determine the heat transfer and drag correlations for any type of porous media, a VAT code can be written to solve the local energy and momentum equations. These equations will give the velocity and temperature distributions of a heat exchanger, which can be used to find the cost effectiveness of a heat exchanger. This fast running VAT tool can find more optimal results than other methods in less time. 
With closure of the friction factor and the heat transfer coefficient, the problem is closed and the porous media governing equation derived from VAT are

$$
\tilde{M}\left(\langle m\rangle, S_{w}, c_{d}\right) \quad \tilde{T}_{s}\left(\langle m\rangle, S_{w}, h\right) \quad \tilde{T}_{f}\left(\langle m\rangle, S_{w}, h\right)
$$

where $\tilde{M}$ stands for averaged momentum equation variables. From the statements above, the macro scale equations are functions only of porous media morphology, represented by porosity and specific surface area, and its closure. Further it was shown that by proper scaling, closure is a function of the porous media as well, which further generalizes macro scale porous media equations. The simple applications described in this work show that multiple parameters can be dealt with and that heat sinks and heat exchangers have a great deal of room for improvement.

Future work would be to incorporate the use of a genetic algorithm directly into the VAT code, an optimal design can be predicted for given constraints. More geometry need to be tested to find more closure correlations that may yield a higher heat transfer from the solid to the fluid, be it on the air side or in the tubes. The special effects of geometry, such as elliptic tubes or augment plane fins would both allow for a more optimal design. 


\section{References}

1. Anderson, T.B. and Jackson, R., 1967, "Fluid Mechanical Description of Fluidized Beds. Equations of Motion",Industrial \& Engineering Chemistry Fundamentals, vol.6, no.4, pp.527-539.

2. Bejan, A. and Kraus, A., "Heat Transfer Handbook", John Wiley \& Sons, 2003.

3. Burke, S.P. and Plummer, W.B., 1928, "Gas Flow through Packed Columns1", Industrial \& Engineering Chemistry, Vol.20, No.11, pp.1196-1200.

4. E. Frank, K. Vajen, A. Obozov, V. Borodin (2006): Preheating for a District Heating Net with a Multicomponent Solar Thermal System, Proc. EuroSun 2006, Glasgow

5. Goldberg, David E. Genetic Algorithms in Search, Optimization, and Machine Learning. 1 edition, Addison -Wesely Proffesional, January 1989

6. Gray, W.G., Leijnse, A., Kolar, R.L. and Blain, C.A., 1993, Mathematical Tools for Changing Spatial Scales in the Analysis of Physical Systems, CRC Press, Boca Raton

7. $\mathrm{Hu}, \mathrm{K}$. "Flow and heat transfer over rough surfaces in porous media", $\mathrm{PhD}$ Thesis, University of California, Los Angeles, 2002.

8. Kang, H. J., Li, W., Li, H. Z., Xin, R. C. and Tao, W. Q.,"Experimental study on heat transfer and pressure drop characteristics of four types of plate fin-and-tube heat exchanger surfaces", Journal of Thermal Science, 1994. 3(1): p. 34-42.

9. Kheifets, L.I. and Neimark, A..V.., 1982 ,"Multiphase Processes in Porous Media", Khimiya, Moscow, pp.320.

10. Lyons, A.M.; Krishnan, S.; Mullins, J.; Hodes, M.; Hernon, D. Advanced Heat Sinks Enabled by Three-Dimensional Printing, Twentieth Annual International Solid Freeform Fabrication Symposium, August 3-5, 2009, Austin, Texas, USA. pp. 749-760.Morcom, A.R., 1946, "Fluid Flow Through Granular Materials", Chemical Engineering Research and Design, Vol.24, pp.30-43.

11. Morcom, A.R., 1946, "Fluid Flow Through Granular Materials", Chemical Engineering Research and Design, Vol.24, pp.30-43.

12. Orozaliev, J., Christian Budig, Klaus Vajen . "OPTIMIZATION OF THE FIN-AND-TUBE HEAT EXCHANGER DESIGN FOR NON-STANDARD APPLICATIONS" Institute of Thermal Engineering, Kassel University, Kassel, Germany. Proceedings of the 14th International Heat Transfer Conference, August 8-13, 2010, Washington, DC, USA. IHTC14-23390

13. Rich, D.G., "The effect of fin spacing on the heat transfer and friction performance of multirow, smooth plate fin-and-tube heat exchangers", ASHARE Trans., 1973, 79(2): p. 135145.

14. Rodi, W., 1984, Turbulence Models and Their Applications in Hydraulics- a State of the Art Review, International Association for Hydraulic Research

15. Slattery, J.C., 1980, Momentum, Energy and Mass Transfer in Continua, Krieger, Malabar

16. Tang, L. H., Min, Z., Xie, G. N. and Wang, Q. W. "Fin Pattern Effects on Air-Side Heat Transfer and Friction Characteristics of Fin-and-Tube Heat Exchangers with Large Number of Large-Diameter Tube Rows", Heat Transfer Engineering, 2009, 30(3): p. 171 - 180.

17. Techo, R., Tickner, R.R. and James, R.E. "An Accurate Equation for the Computation of the Friction Factor for Smooth Pipes for the Reynolds Number", J. Appl. Mech., 1965, 32: 443. 
18. Travkin V.S. and Catton, I.,1992, Models of turbulent thermal diffusivity and transfer coefficients for a regular packed bed of spheres. In Fundamentals of Heat Transfer in Porous Media, ASME HTD 193 pp 15-23.

19. Travkin, V. S., Gratton, L. and Catton, I., "A Morphological Approach for Two-Phase Porous Medium-Transport and Optimum Design Applications in Energy Engineering", Proc. 12th Symp. Energy Engin. Sciences, Argonne National Laboratories (1994)

20. Travkin, V. S. and Catton I. "A Two-Temperature Model for Turbulent Flow and Heat Transfer in a Porous Layer", Journal of Fluids Engineering, 1995, 117(1): 181-188.

21. Travkin, V. S. and Catton I., "Porous media transport descriptions -- non-local, linear and non-linear against effective thermal/fluid properties", Advances in Colloid and Interface Science, 1998, 76-77: 389-443.

22. Travkin, V. S., Serglevsky, E.D., Krinitsky, E.V. and Catton, I., "Integrated Heterogeneous Design of Semiconductor Heat Sink via Scaled Direct Micro-Modeling, Upper scale VAT Simulation and Experiment. Comparison and Verification of Properties", Proceedings of 2001 ASME IMECE, New York.

23. Vadnjal, A. "Modeling of a Heat Sink and High Heat Flux Vapor Chamber", Aerospace Engineering, PhD Thesis, University of California, Los Angeles, 2009

24. Wang, C. C., Chang, Y. J., Hsieh, Y. C. and Lin, Y. T. "Sensible heat and friction characteristics of plate fin-and-tube heat exchangers having plane fins", International Journal of Refrigeration, 1996, 19(4): p. 223-230.

25. Wang, C. C., Chi, K. Y. and Chang, C. J. "Heat transfer and friction characteristics of plain fin-and-tube heat exchangers, part II: Correlation", International Journal of Heat and Mass Transfer, 2000, 43(15): 2693-2700.

26. Whitaker S., 1967, "Diffusion and dispersion in porous media", AIChE Journal, Vol. 13, No. 3, pp. $420-427$.

27. Whitaker, S. "Forced convection heat transfer correlations for flow in pipes, past flat plates, single cylinders, single spheres, and for flow in packed beds and tube bundles", AIChE Journal, 1972, 18(2): 361-371.

28. Whitaker, S. "Flow in porous media I: A theoretical derivation of Darcy's law", Transport in Porous Media, 1986, 1(1): 3-25.

29. Whitaker, S., 1999, The Method of Volume Averaging, Kluwer Academic Publishers.

30. Xie, G. N., Wang, Q. W. and Sunden, B. "Parametric study and multiple correlations on airside heat transfer and friction characteristics of fin-and-tube heat exchangers with large number of large-diameter tube rows", Applied Thermal Engineering, 2009, 29(1): p. 1-16. 